NASACONTRACTOR REPOR T

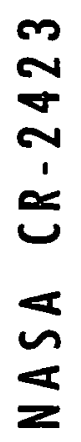

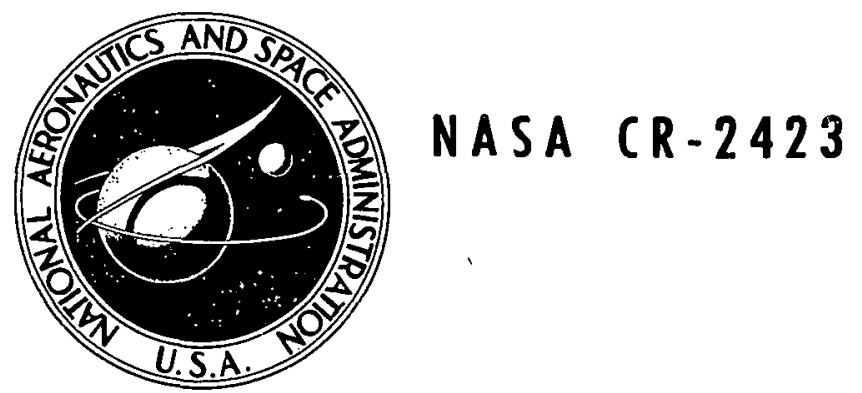

\title{
AERODYNAMIC INFLUENCE COEFFICIENT METHOD USING SINGULARITY SPLINES
}

by J. E. Mercer, J. A. Weber, and E. P. Lesferd

Prepared by

THE BOEING COMPANY

Seattle, Wash. 98124

for Ames Research Center

NATIONAL AERONAUTICS AND SPACE ADMINISTRATION - WASHINGTON, D. C. - MAY 1974 


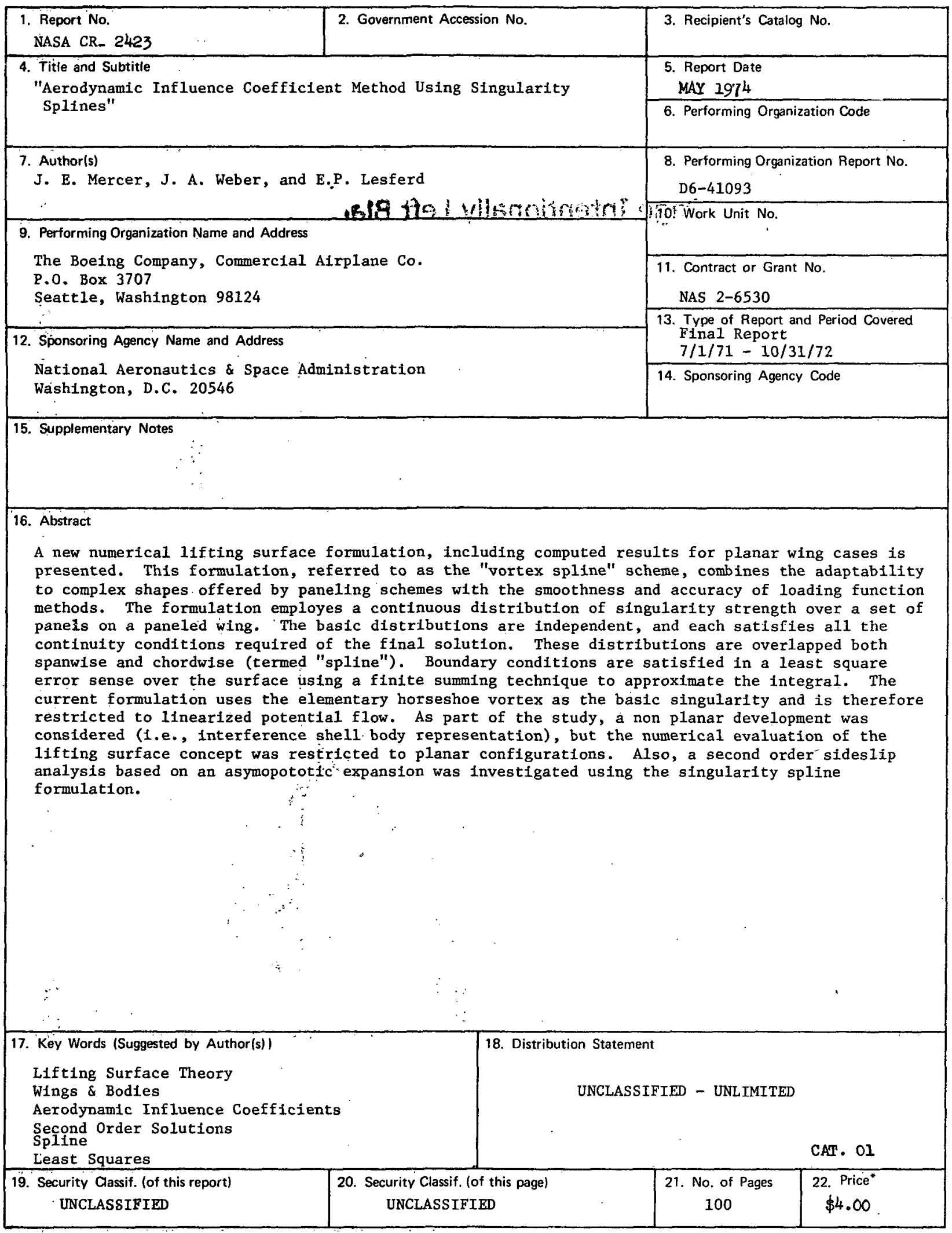

* For sale by the National Technical Information Service, Springfield, Virginia 22151 
CONT ENTS

1. SUMMARY 1

2. INTRODUCTION 2

3. NOHENCLATURE 4

4. REQUIREMENTS FOR NUMERICAL SCHEME

b. VORTEX SPLINE SCHEME 12

b. 1 General Approach 12

5. 2 Selection of Vorticity Distribution 17

- Two-Dimensional Study 17

o Basic Three-Dimensional Punction 22

- Modeling Vorticity on a ring Using

- Ning Paneling 38

5.3 Evaluation of Kernel Function Integrals 42

5. 4 Satisfaction of Boundary conditions on
Singularity surfaces.

5.5 Computational Procedure $\quad 63$

5.6 Numerical Results $\quad 65$

6. SHEL REPRESENTATIOA USING THE SPLINE SCHEHE 74

6. 1 General Approach 74

6. 2 Interference Shell Singularities 76

7. SECOND ORDEB SOLUTIUNS USING THE VORTEX SPLINE 85

8. CONCLUDI HG REMARKS

$\begin{array}{ll}\text { 9. REFER ENCES } & 91\end{array}$

10. APPENDIX - Doublet Characteristic Box Method 93 
F IGU EES

Eigu도오

pagge

Edge Conditions for Supersonic Plantorms 11

2. Axis system for Flow modeling 14

Constant pressure panel Scheme in Tro Dimensions, 18

Two-Dimensional Constant Pressure Panel Results . 20

Linear Vorticity Representation Osed Chordyise

21

6

Two-Dimensional Spline Results

Location of Dounuash Singularities vith Vortex spline

8 Quadratic Distribution Formed from linear Function and Quadratic Additions

Surface spline Distribution of Vorticity..:

Spline Punctions Distributed on a wing (syanetric Flow)

Antisymetric Spanvise Spline Distribution

Overlapping of Surface Distributions

13 Quadratic Panel Representation Along a Planfor Break

Borvard Mach Cone on the Surface of a ding

Typical Forward Mach Line Intersections

vith a panel

Logic Block to set limits of Supersonic Integrals

partitioning of AIC Matrix for Mach Line Paneling

Triangular Pressure Distribution Model of a Plat Plate at Angle of Attack 
24 : Tapered Hing with zero sweep of Quarter chord

Various panelings of a circular Hing

31 Paneling of an Interference she 11

32 Three panel spline runctions on Interference shell 
AERODYNAMIC INFLUENCE COEFFICIENT METHOD

USING SINGULARITY SPLINES

By J. E. Mercer, J. A. Weber

and E. P. Lesferd

The Boeing Company

\subsection{SUMMARY}

A new numerical lifting surface formulation, including computed results for planar wing cases is presented. This formulation, referred to as the "vortex spline" scheme, combines the adaptability to complex shapes offered by paneling schemes with the smoothness and accuracy of loading function methods. The formulation employs a continuous distribution of singularity strength over a set of panels on a paneled wing. The basic distributions are independent, and each satisfies all the continuity conditions required of the final solution. These distributions are overlapped both spanwise and chordwise (termed "spline"). Boundary conditions are satisfied in a least square error sense over the surface using a finite summing technique to approximate the integral. The current formulation uses the elementary horseshoe vortex as the basic singularity and is therefore restricted to linearized potential flow. As part of the study, a non planar development was considered (i.e., interference shell body representation), but the numerical evaluation of the lifting surface concept was restricted to planar configurations. Also, a second order sideslip analysis based on an asymptotic expansion was investigated using the singularity spline formulation. 


\subsection{INTRODUCTION}

various lifting surface schemes bave been proposed for.. the analysis of arbitrary wing planforms. Most of these methods traat this fluid dynamic problea by relating one flow paraweter to another (e.g.. downwash to pressure jump). A majority of the apuroaches can be characterized as constrlcting a solution from a composite of basic, assumed distributions of fressure. vorticity, or potintial, all of wich satisfy the governing differential equation. The boundary conditions of the problem determine the relative magnitudes of the various distributions. "eact of these assumed distributions can cover the entire lifting surface or merely portions of it, depending on the particular numerical scheme.

A lthough many of the schemes arise from the above described common origin, in practice there can be great differences among them. As an example, consider the constant pressure panel (Ref. 1) and pressure mole (Ref. 2) schemes. The constant pressure panel scheme uses a single type of distribution that is of constant pressure ranging over some small region:of a. wing. A solution using this method is one that consists of a set of steps in the pressure level which can only roughly apfroximate the true solution. However, the method is applicable to both subsonic and supersonic tlovs and ranges over a wide variety of planform shapes. The pressure mode scheme, on the other hand, uses several different types of distributions ranging cver all or at least a large portion of a wing. The solution using this method can yield very accurate pressure results. However, the method requires careful attention to discontinuities in supersoric flo and it is restricted in the geometry of planforms which it can treat. In choosing between these two schemes, trades between pressure details and generality of configurations must be weighed.

The FLEXSTAB system (Ret. 3) which pertorms the stability and control analysis of elastic arbitrary wing-body configurations was developed using the constant pressure panel schene because cf the schemes planform generality. Using this aerodynamic method, the program. generates rot only symetric stability derivatives (e.g., $\mathrm{C}_{\mathrm{L}_{\alpha}}, \mathrm{C}_{\mathrm{M}_{\alpha}}$ et $\left.\mathrm{C}_{-}\right)$, but also antisymetric statility derivatives (e.g. ${ }^{C_{C_{B}},} \mathrm{C}_{\mathbb{N}_{B}}$, etc.). It was soon recognized, however, that configurations with small ving dihedral could not be accurately analyzed for certain of their antisymmetric derivatives, specifically the rolling moment derivative, $C_{l_{B}}$. Upon examination, it was discovered that the accuracy of the constant pressure panel scheme was not adequate for the analysis of these configurations. In an attempt to establish the requirements necessary to troat such cases, a study was performed (Ref. 4). This study, based on an asymptotic expansicn of the differential equation in terms of the governing flow variables (E.g., angle-of- 
attack and angle of sideslipl. revealed that gradients of the pressure distribution appear to play as active a role in the sideslip problem of configurations vith small dihedral as the pressures themselves. This result confirmed the belief. that" the constant pressure panel scheme could not treat this problem. Furtber investigation of other lifting surface schemes which might be applicable was initiated. This study concluded that no existing schene could provide the needed pressure gradient accuracy required for the small dihedral configurations and at the same time, provide the desired generality. Accordingly, research to create a new scheme was begun. The cesult of this study was the development of the "vortex spline" scheme: a method which satisfies the above mentioned requirements. The key features. cf this method are:

1) The use of independent, overlapping singularity distributions wich are arranged such that the final solution for the pressure ill automatically be continuous in both value and slope (unless specifically required not to be at certain locations)

2) The use of ying paneling to frovide a basis for the extent of coverage for each distribution, thereby giving it the generality of the constant fressure panel method

3) The satisfaction of boundary conditions using a least square error technique

4) The application to both subsonic and supersonic flow

5) The use of "special Mach lines" for paneling in supersonic flow.

The curcent formulation of the schewe is restricted tc linearized, steady, potential flcw. The restriction to linearized flow is due mainly to the use of the elementary horseshoe vortex as the basic singularity strength. This singularity was selected because of its automatic treatment of the linearized vake boundary condition and its compatability with the existing Flexstak aérodynamic representation.

This roport includes: 1) the derivation of the numerical scheme for flat wings. of zero thickness along with computed results, 2) the description of a compatible interference shell representation (Ref. 1), 3) the description of a second order numerical scheme to compute interaction effects such as those which domjnate for small dihedral, and 4) an alternate supersonic scheme studied initially (included in the afpendix). 


\section{$\because \therefore \therefore \quad 3: 0:$ NOMENCLATURE}

$\mathbf{a}$

${ }^{A_{C}}$

al

$A_{1 j}$

$A_{\text {L }}$

${ }^{A} M$

$A_{0}$

$\tilde{A}^{\mathrm{T}}$

b

B II, I

c

$\overline{\mathbf{c}}$

$c(n)$

$\mathrm{C}_{\ell}$

$\mathbf{C}_{\mathbf{L}}$

$\mathrm{C}_{\mathrm{p}}$

$E^{2}$

$G_{!}$

$\mathrm{G}_{2}$

$\overline{\mathbf{G}}$

$\mathbf{K}$

amplitude of quadratic addition to panel edge, also used as a coefficient of the equation for a panel edge.

strength of Cauchy singularity

area associated with ith control point

aerodynamic influence coefficient, an element of [A]

strength of logarithmic singularity

strength of Mangler singularity

strength of non-singular terms evaluated at the singularity.

weighted transpose of influence coefficient matrix.

coefficient of the equation for a panel edge

influence coefficient representing the effect of

Subdivision I on. Subdivision II.

coefficient of the equation for a panel edge

average wing chord

panel chord variation $\left(x_{T E}-x_{L E}\right)$

section lift coefficient

wing lift coefficient

pressure coefficient

square error

integral of the kernel function in the $x$ direction (linear in $x$ variation of vorticity)

integral of the kernel function in the $x$ direction (constant in $x$ variation in vorticity)

numerical representation : of $G_{1}$ and $G_{2}$ kernel function 
Mach number, also, number of control points

$N \quad$ number of spline functions

Ho neutral point

P

$\mathbf{r}$

$R_{\mathbf{J}}$

8

$\mathbf{s}$

$\mathbf{u}$

$\mathbf{U}_{\infty}$

v

w

$w_{1}$

wis

$\mathbf{w}^{\prime}$

$\mathbf{w}_{\mathrm{BC}}$

$\mathbf{w}_{\mathbf{S}}$

$x$

$\bar{x}$

$x^{1}$

$\mathbf{y}$

$\mathbf{Y}^{\mathbf{n}}$

$+n$

pressure

cylindrical coordinate and also Mach line coordinate of Mach line with panel edge)

area of integration and also Mach line coordinate strength associated with function $r_{j}$, an element of $(\mathrm{s})$

perturbation velocity in $x$ direction divided by $U_{\infty}$

free stream velocity

perturbation velocity in $\mathrm{Y}$ direction divided by $U_{\infty}$ perturbation velocity in $z$ direction divided by $U_{\infty}$ downwash on control point, i

downwash on control point $i$, due to spline function, $j$ downwash on downstream subdivision due to upstream subdivision (used in supersonic flow)

downwash specified by boundary conditions

downwash from spline functions

Cartesian coordinate

nondimensional $\times$ coordinate

segment of chordwise vorticity spline variation

( $l$ is an index, not a power)

Cartesian coordinate

segment of spanwise vorticity spline variation ( $n$ is an index, not a power)

$\partial \mathbf{Y}^{\mathbf{n}} / \partial \mathbf{y}$ ( $\mathrm{n}$ is an index, not a power) roots of Mach line - panel edge equation (intersections 
$\overline{\mathbf{y}}$

$z \quad$ Cartesian coordinate

a angle of attack

B

$c_{1}, \varepsilon_{2}, \varepsilon_{3}$

$r$

$r_{j}$

6P

$\Delta \mathrm{C}_{\mathrm{p}}$

$\Delta$ slope $\Delta x_{1}, \Delta x_{2}$

6

$n$

-

-

j

$\theta$

p

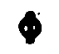

9d

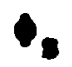

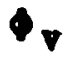

$\Delta$

bound vorticity

perturbation in pressure

jump in panel edge slope

shed vorticity

cylindrical coordinate

density of fluid

jump in potential

$\sqrt{1-M^{2}}$ (subsonic) or $\sqrt{M^{2}-1}$ (super sonic)

small distance from singularity- (used to evaluate $\bar{G}$ )

spline function vorticity distribution

jump in pressure coefficient across wing surface

quadratic additions to panel edges

running coordinate in $\mathrm{x}$ direction

running coordinate in $y$ direction

nondimensional coordinate

circumferential vorticity spline variation

perturbation velocity potential

potential due to a point doublet

potential due to a point source

potential due to an elementary horseshoe vortex 
Matrix Notation

[A] matrix of elements, $A_{1 \mathrm{~J}}$

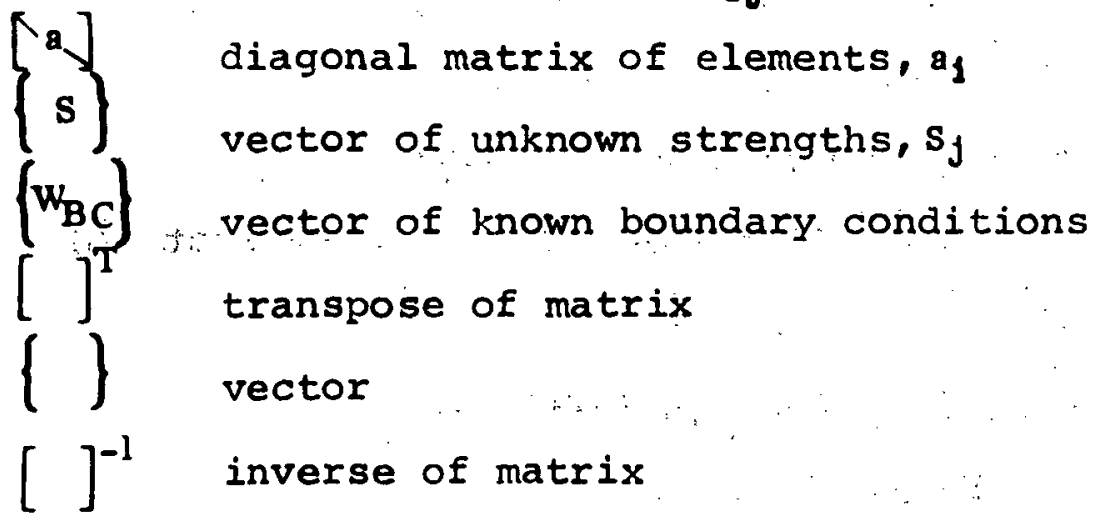

Subscripts

H homogeneous solution to differential equation

1 control point evaluation

$l$ lower surface

L left panel edge

LE leading edge

ML Mach line

- define location of singularity on shell surface

P particular solution to differential equation

$R$ regular (non singular variable) and also right panel edge

s singular variable

TE trailing edge

. upper surface 


\subsection{REQUIREMENTS FOR NUMERICAL SCHEME}

Before developing a new scheme, a set of requirements and desirable features was astablished. The requirements were hased on 1) ultimately incorporating the scheme into FLEXSTAB and 2 ' the analysis performed in reference 4 (the latter because the "main" objective was to model flows around configuratior with small yeometric dihedrail): The desirable features were based: on axperience ith various lifting surface schemes.

The requirement set forth in reference 4 was that any numerical shcem that is to be used for interaction flo analysis (e.g.. sideslip-angle of attack) nust be capable of predicting yradients of pressure to the same degree of accuracy ais most techniques currently predict pressure levels. This implies that the basic equivalent pressure distribution used to construct the solution must vary linearly as a minimum. A linear variation could appoximate pressure gradients in the same way the constant pressure panel schemo approximates fressure levels.

A Eurther requirement, established by the ultimate desire to incorporate the final result into FlEXSTAB, was that the new technique must be adaptable to arbitrary planforms.: irom experience with both pressure mode techniques and paneling techniques it was felt that treatment of arbitrary configurations can best be achieved through a paneling technique. Such techniques divide a configuration intc many parts (called paneis). A distribution of flow singularities is associated with each fanel and therefore, these singularities contorm to very complex geometries by mere adjustments to the panel sizes. shapes, and orientations.

Experience using various numerical schemés also showed that difficulties arise when attempting to model a lifting surface wich is in the vake of another (E.G., a tail behind a wing): the source of the froblem is singularities in the downash field inherent in various numerical formulations. Because of these singularities, the downush influence is only correct at discrete points in the flow field. "Fcr Faneling techniques (which constitute the major source of this problem) this means that the trailing surface's paneling is determined by the leading surface's paneling or vice versa (which ever provides the more stringent requirement).. When dealing. with arbitrary configurations many such paneling problems can arise thus causing much time to be spent establishing the paneling arrangement and in manycases, causing more panels to be used than would be for methods that do not produce downash singularities. This was not considered acceptable, therafore the requirement was established to eliminate as many of these singularities as practical. 
As a desirable feature, it was felt that a basic scheme applicable to both subsonic and supersonic flcw would be ideal. Although not a requirement, such an arrangement could reduce the coding needed to implement the scheme and at the same time allow a single mathematical model to be analyzed all the way from incompressible fioy through the supersonic regime.

Finally, the most desirable general possitle approach would be a single basic scheme which could be used for both analysis and design. This again would greatly reduce coding and at the same time permit the user to almost arbitrarily switch analysis and designtunctions wile designirg an aircratt. As an example, a designer could generate a shape for a given flight condition and then analyze tris configuration "off design" a11 in one run greatly speeding. up the design process.

Summarizing then, the general requirements are:

1) Capability to dodel pressure gradients

2) Arbitrary planform modeling

3) Minimum downwsh sinqularities in flow field

The geaeral desiratle features are:

1) Unified approach for both subsonic and supersonic flow

2) A single basic scheme for totb design and analysis.

Betore any development of a new scheme vas begur, a study of the characteristics of both subsonic and supersonic flow was undertaker to establish some of the more detailed numerical considerations. For subsonic linearized flow the properties can be summrized as:

1) A singularity in the fressure cr bound vorticity at the leading edge wich varies as the recifrocal of the square root of the distance from the edge - this causes the flow tc turn abruptly at the leading edge.

2) The kutta condition at the trailing edge (vanishing bound vorticity or pressure) - this is required to make the analysis mathematically unique and physically arises from viscous effects.

For supersonic, linearized flow there are several features to be considered: 
1) Hyperbolic nature, - a solution can proceed from fore to aft on a configuration.

2) Limited regions of influence - disturbances are limited to the interior of Mach cones and tend to concentrate most strongly along Mach cone boundaries:

3) Differing edge conditions - depending ou the steep of an edge with respect to a Mach line, the character of the solution differs: supersonic leading edges exhibit regular behavior whereas subsonic ones show a singular behavior. subscnic trailing edges require a kutta condition, whereas supersonic ones do not.

4) Discontinuous nature of flow across "special Mach lines" (those emanating from planfcrm edge breaks - Fig. 1) - along special Mach lines the surface pressure and vorticity distribution can exhibit discontinuous behavior in levels and for gradients. Within regions bounded by these special Mach lines the flow exhibits a smooth regular behavior.

various schemes use different means to represent the above characteristics and in many cases the characteristics are only approximated; yet, these approximations yield adequate results. Therefore, it is not an absolute modeling reguirement that a new aumerical formulation possess the ability to exactly represent all of the above characteristics: it is only necessary to carefully study, the impact of each in the evaluation of the scheme.

The next section contains the analysis used to develop a new numerical scheme, the vortex spline, along.with the comfuted results. The appendix contains another method for supersonic flow only, the doublet characteristic box, which was investigated but not fully developed. 

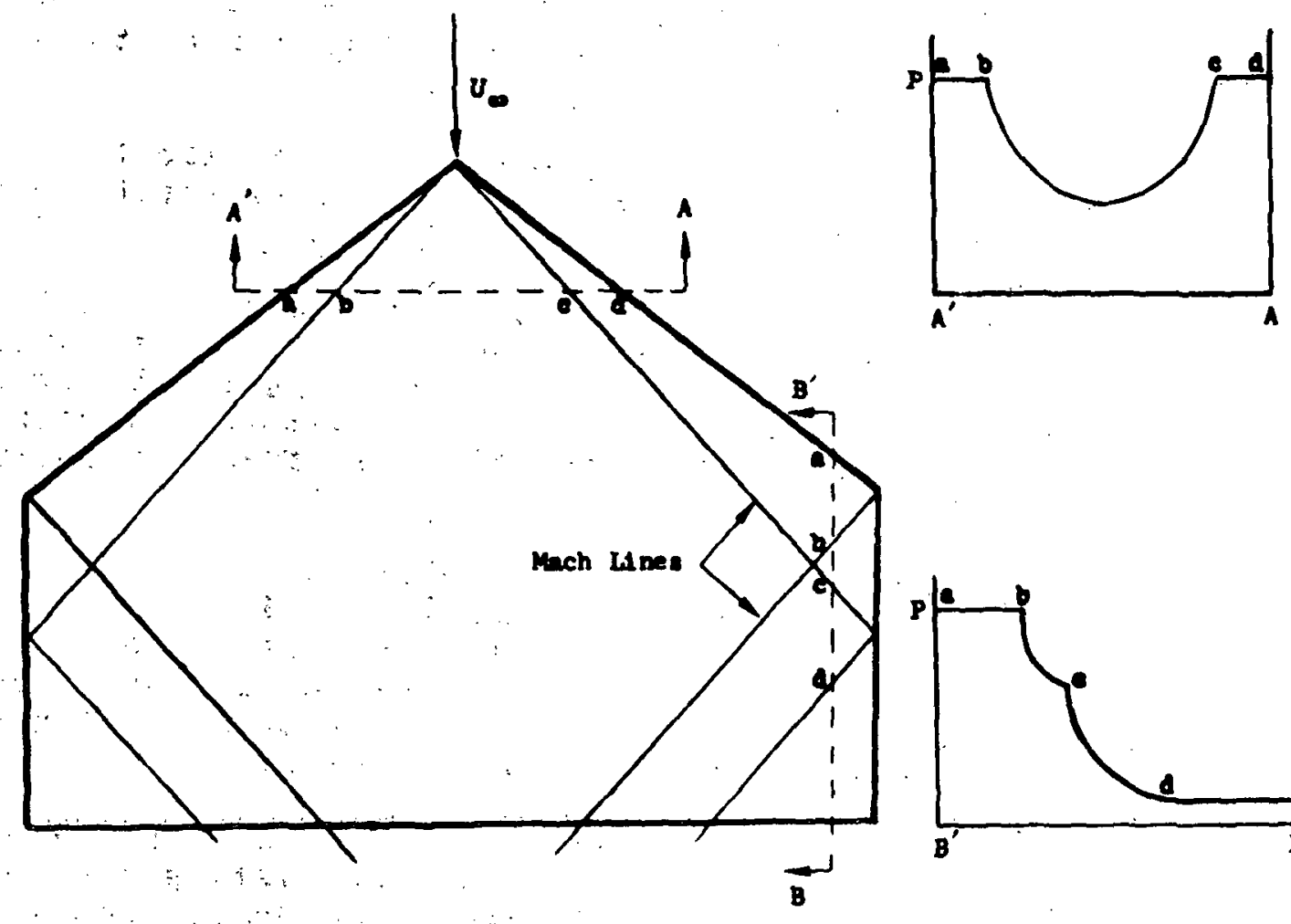

(a) Supersontc Leading bage
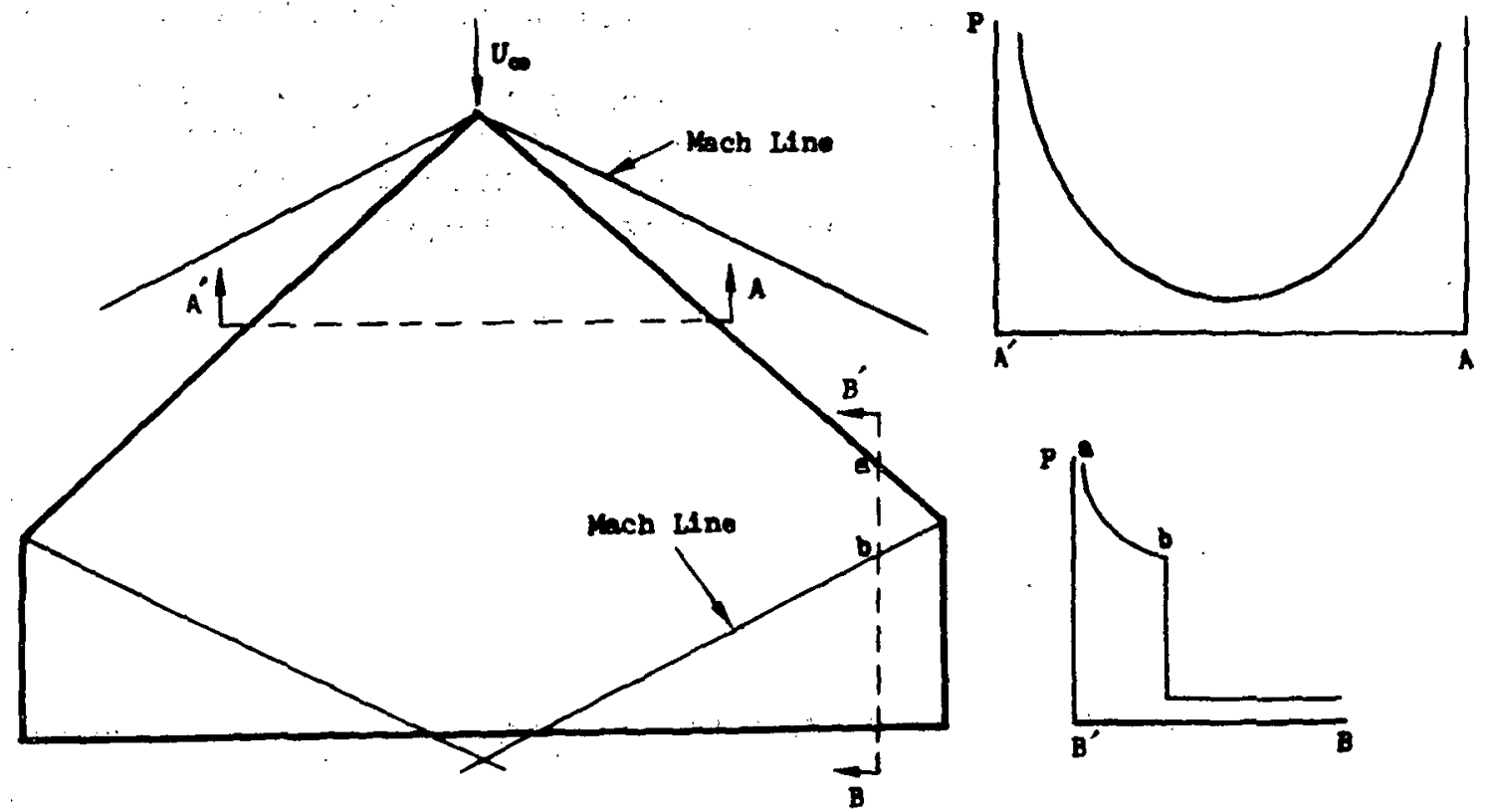

(b) Subsonic Leeding Bagee

FIGURE 1 - EDGE CONDITIONS FOR SUPERSONIC PLANFORMS 


\subsection{General Approach}

As outlined in the introduction, most lifting surface schemes model flow problems by relating one flow parameter to another. For a nalysis problems the known parameter is the vehicle shape while the unknow is the pressure distribution. Since both the known and unknown parameters can be expressed in terms of velocity and velocity related parameters the forthcoming analysis will be derived using these variables.

Assuming linearized flow, the perturbation pressure can be expressed as:

$$
8 P=-P_{\infty} U_{\infty} \delta u
$$

where ou is the perturbation velocity in the free stream direction with $U_{\infty}$ being the free stream velocity. Dividing Equation (1) by the dynamic fressure, $\frac{1}{2} P_{\infty} U_{\infty}^{2}$. gives:

$$
\frac{8 P}{1 \rho_{\infty} U_{\infty}^{2}}=-2 \frac{8 u}{U_{\infty}}
$$

or

$$
c_{p}=-2 u
$$

where $c_{p}$ is the pressure coefficient and $u=\delta u / U_{\infty}$ is a nondimensional perturbation velocity.

Across a lifting surface there is a jump in pressure, $\Delta C_{p}$. corresponding to a jump in the perturbation velocity, u. jump can be expressed in terms of another velocity related parameter - the bound vorticity, $r$ :

$$
r \equiv u_{u}-u_{l}
$$

where $u_{u}$ is the upper surface velocity perturbation ard $u_{l}$ is the lover surface velocity perturbation. 
since $r$ correspunds to an antisymetric shear across the lifting surtace (Bef. 5, pg. 129)

$$
u_{u}=-u_{l}=u
$$

therefore:

$$
r=2 u
$$

and:

$$
\Delta C_{p}=2\left(u_{u}-u_{l}\right)=4 u=2 r
$$

From Eqution (7) it is clear that the jump in pressure across a surface, assuming linearized oflow, is directly related to the shear flow, parameter - bound vorticity. $r$.

on a two-dimensional planar surface, there are two components of velocity wich are discoritinuous, $u$ and $v$; there are correspondingly two components of vorticity - $r$ corresfonding to $u$ and $\sigma$ corresponding to $v$ (see Ref. 6, py. 22.1):

$$
0 \equiv-\left(v_{u}-v_{l}\right)=-2 v
$$

where $v$ is the nondimensional, perturbation velocity normal to the free streall.

I ${ }^{\prime}$ troating potential flow, o and $r$ are not independent and can be related through Helmholtz theorem (Ref. ó, pg. 221) as (Pig. 2) :

$$
\frac{\partial 0}{\partial x}+\frac{\partial \gamma}{\partial y}=0
$$

An plementary solution to the yoverning ditferential equation:

$$
\left(1-M^{2}\right) \frac{\partial^{2} \phi}{\partial x^{2}}+\frac{\partial^{2} \varphi}{\partial y^{2}}+\frac{\partial^{2}}{\partial z^{2}}=0
$$




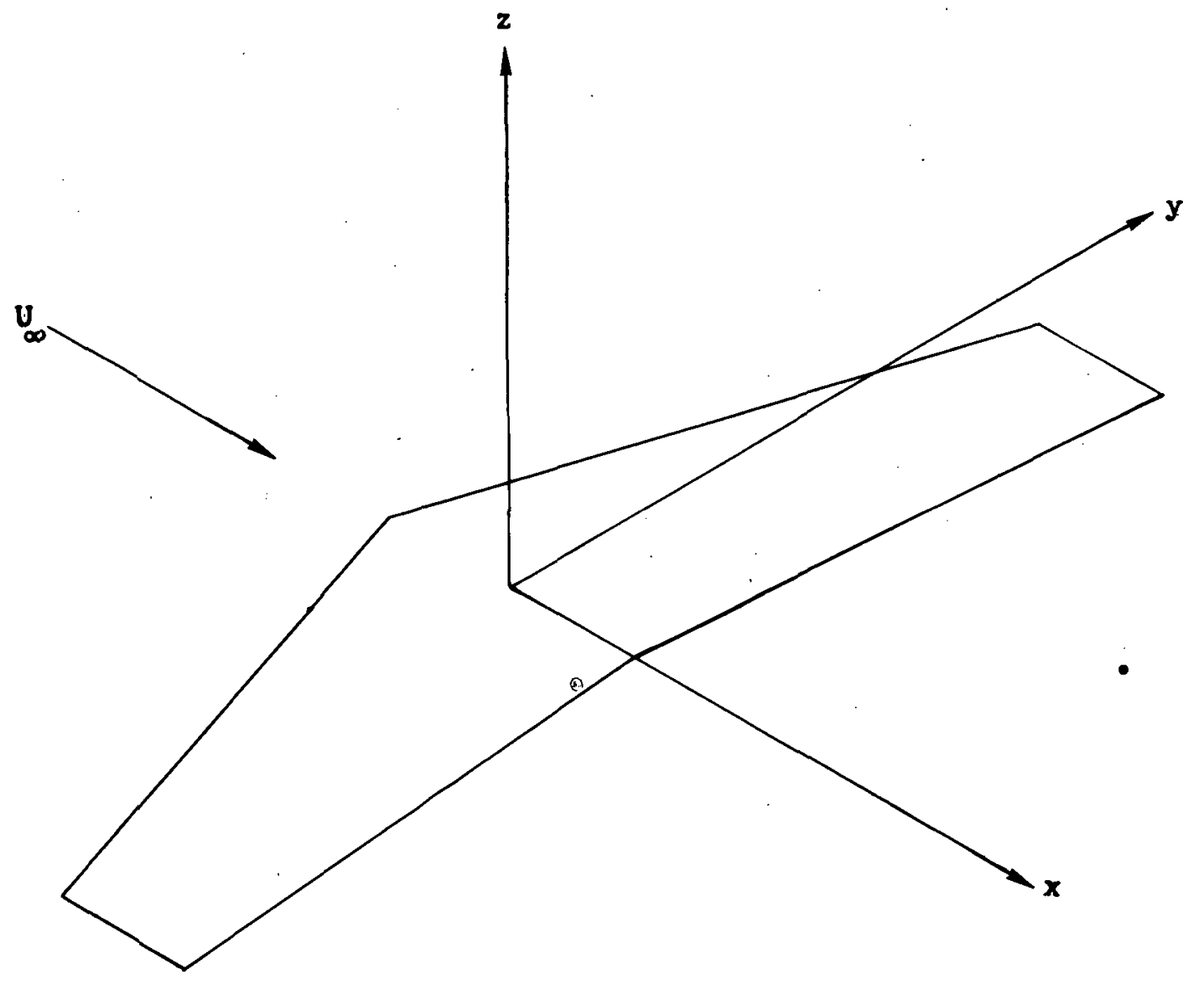

FIGURE 2 - AXIS SYSTEM FOR FLOW MODELING 
which produces shear flow corresponding to vorticity can be obtained by integrating the basic doublet (Ref. 5). This solution tormed the "elementary horseshce vortex" automatically satisfies the linearized wake boundary condition of a jump in pctential but no jump in pressure.

Using the elementary horseshoe "ortex as the basic singularity, planar flow problems can be modeled as follows:

$$
w(x, y)=\iint_{B} r(5, n) K(6-x, n-y) d \zeta d n
$$

where $w(x, y)$ is the known nondimensional downwash velocity (for small angles $w$ is approximately a, the angle of attack), $x$ and $y$ are field points, $s$ is the region over which the singularities are distributed, $\quad r(\zeta, n)$ is the unknown bound vorticity distribution, and $k(\xi-x, n-y)$ is the horseshoe vortex kernel function.

For subsonic flov, the region of influence, 8 , and the entire singularity surface coincide. The kernel function for this flow regime is (Ref. 5, pp. 87 and 149):

$$
K(6-x, n-y)=\frac{1}{4 \pi} \frac{1}{(n-y)^{2}}\left[1-\frac{6-x}{\sqrt{(5-x)^{2}+\beta^{2}(n-y)^{2}}}\right]
$$

where $B^{2}=i-M^{2}$.

For supersonic flow, the region of influence, $s$, is the portion of the singularity surface contained in the forward Mach cone emanating from the field point $(x, y)$. The kernel function for this flow regime is derived in the same manner as was done for subsonic flow (Ref. 5, pg. 87) except that the analysis begins with the supersonic source (Ref. 5, pg. 88). Since the supersonic singularities can only influence locations within the aft Mach cone, the integration along the line of doublets does not range from $x$ to $\infty$ but rather from $x$ to the intersection of the line of doublets with the forward Mach cone from the influenced foint (i. $3 . . x-\beta \sqrt{(n-y)^{2}}$ for a doublet line in the $z=0$ plane). The resulting kernel function is:

$$
K(\zeta-x, n-y)=-\frac{1}{2 x} \frac{1}{(n-y)^{2}} \frac{\zeta-x}{\sqrt{(\zeta-x)^{2}-B^{2}(n-y)^{2}}}
$$

where $\beta^{2}$ is defined as $M^{2-1}$ for supersonic flow. 
As mentioned earlier, $r(\zeta, n)$. the unknown, cian be represented in an approximate way as a series of basic distributions $Y_{j}(r, n)$, so that:

$$
r(s, n)=\sum_{j=1}^{N} s_{j} r_{j}(s, n)
$$

where $s_{j}$ is the strength of the $j^{\text {th }}$ distribution. This terhnique permits the integration tc be performed using the known distributions of $Y_{f}(\zeta, n)$; thereby reducing the problem to an algebraic solution for $s_{j}$. The algebraic formulation is established by specitying the downwash at M $\geq N$ "control points" (i.e., collocation points):

$$
w\left(x_{1}, y_{1}\right)=w_{1} \quad 1=1, \ldots M
$$

where. W is the downash at $x_{1}, y_{1}$ ' 'Substituting Equation (14) into Fquation (11) and integrating yields:

$$
w_{1}=\sum_{j=1}^{N} A_{1 j} s_{j} \quad i_{1} \ldots \ldots
$$

The aerodynamic influence coefficient, $A_{1} f=\frac{\partial w_{1}}{\partial S_{j}}$, represents the integral in Equation (11) (with $s_{j}=1$ ) and gives the downwash at point $i$ due to unit strength of the jthdistribution of vorticity. Iquations (16) are a set of algebraic equation fcr $s_{f}$. For equal to $N$, Equations (10) are a determinate set of ligebraic expressions with a unique solution for the $S_{f}$. For $M$ greater than $N$ there are more equations than unknowns and. as will be shown later, the $S_{j}$ can be determined in a least square error sense. Once the $s_{f}$ are computed the pressures and pressure jumps can be computed trom Equation (7).

Lifting surface schemes using the horseshoe vortex kernel, differ in the form of the basic distribution, $y_{j}(5, n)$ that they assume. They also differ in the manner in which the boundary conditions are satistied, through the location of the field fcints and/or the numbir used in relation to the number of unknowns. The next section describes the type of vorticity assumed for the vortex spline scheme. 
o Tro-Dimensional study

Before attempting a full three-dimensional study, various numerical techniques were first developed in twodimensions. These two-dimensional studies supplied insight while at the same time providing a fundamentally simpler means to obtain numerical results than woula be required for the full three-dimensional problem. The initial investigation concerned various vorticity distributions. In two dimensions, the basic vorticity kernel functicn for downasth is:

$$
K(x)=\frac{1}{2 \pi} \frac{1}{x-5}
$$

therefore the downwash is:

$$
w(x)=\frac{1}{2 \pi} \int_{x_{1}}^{x_{2}} \frac{r(5)}{x^{-\zeta}} d \zeta
$$

A study was adde using this : expression as a twodimensional formulation of the constant pressuce panel scheme. With $r(5)=1$ for $x_{1}<5<x_{2}$ the integration can then be performed yielding:

$$
v(x)=\frac{1}{2 \pi} \ln \left|\frac{x-x_{1}}{x-x_{2}}\right|
$$

Pigure 3 shovs how the schere is used to model the loxding on a flat plate at angle of attack.

The vorticity distribution is a series of step functions. The influence of the $j^{\text {th }}$ step tunction, having strength, $S_{j}$, on the $i^{\text {th }}$ control point can be expressed from Fquation (19) as:

$$
w\left(x_{1}\right)_{j}=w_{1 j}=\frac{1}{2 \pi} s_{j} \ln \left|\frac{x_{1}-x_{j}}{x_{1}-x_{j}+1}\right|
$$

or

$$
w_{1}=A_{1 j} s_{j}
$$



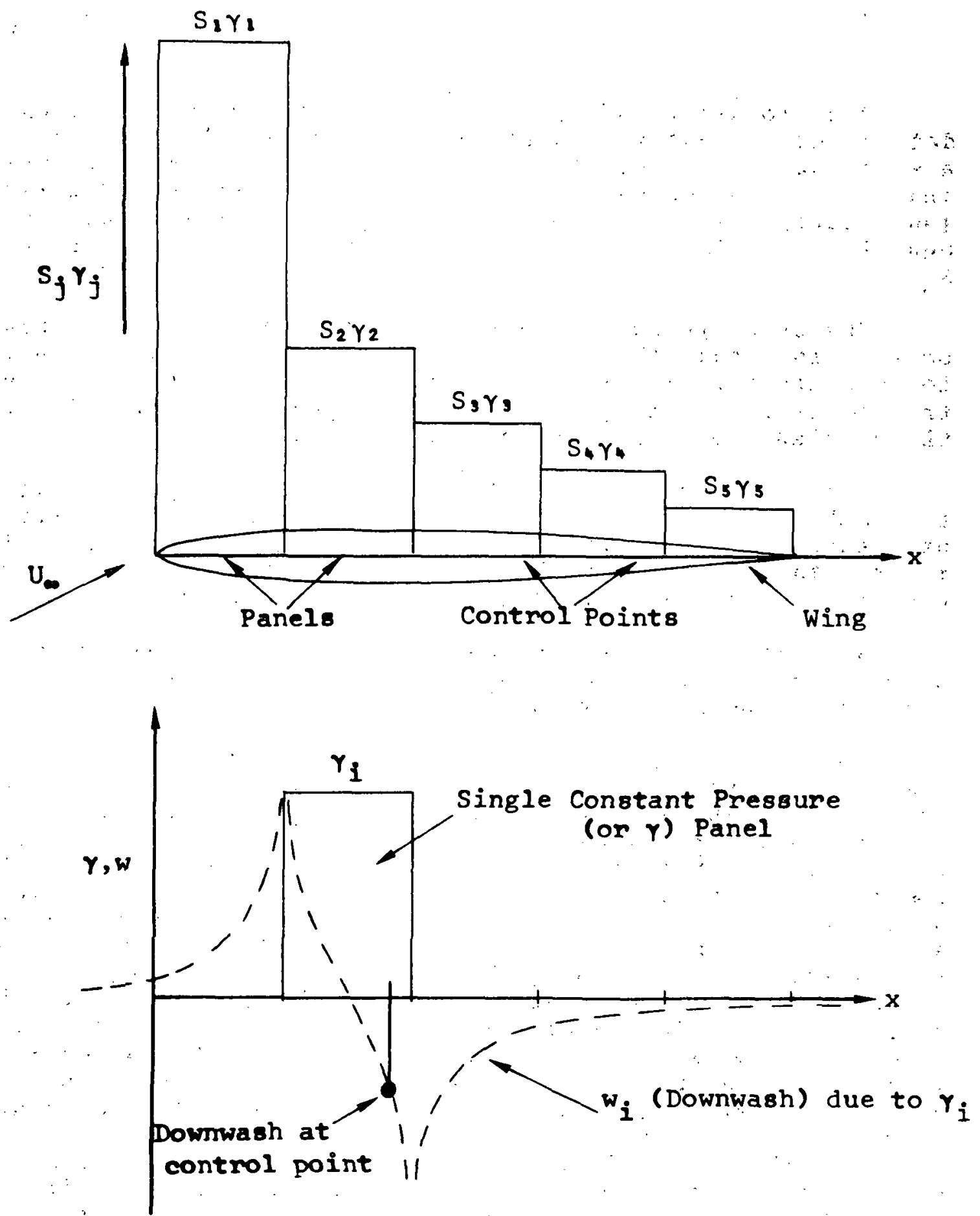

FIGURE 3 - CONSTANT PRESSURE PANEL SCHEME IN TWO DIMENSIONS 
where

$$
A_{1 j}=\frac{1}{2 \pi} \ln \left|\frac{x_{1}-x_{j}}{x_{1}-x_{j}+1}\right|
$$

The location of the control points is computationally determined so that the numerical results will be correct over a wide range of problems. Since there is one control point and one value of $S_{j}$ corresponding to each panel. Equations (16) constitute a determinate set of linear algebraic equations $(M=N)$ which can easily be solved for the strengths s $\mathrm{j}$

The thing to note about the constant pressure panel scheme is that the downash field for the panels contain a logarithmic singularity at each panel edge. This means that the control point placement on the panel is critical since the gradients of the downash can be large.

Figure 4 shows the results of the flat plate analysis for subsonic flow. These results show the large downash gradients and also show that there is no way to specifically. invoke the kutta condition at the trailing edge. This condition is implied through the control point location.

In spite of these problems the results are fairly good; but, however, they are not sufficient for the interaction problem (as pointed out in the introduction).

In order to improve upon these results it vas apparent that two things had to be done - 1) remove as many of these logarithmic sinqularities as practical, and 2) establish some definite means to introduce the kutta condition in subsonic flow. The first item was achieved by noting that the logarithmic singularities arise from jumps in the vorticity strength therefore a new distribution had to be formulated wich had continuity of vorticity level. This inplied that the vorticity had to posess at least a linear variation (consistent with the requirements set forth in Ref. 4 , and outlined in the Introduction). There are two means to obtain a continuous distribution using a linear distribution - one is to place separate continuity constraints on the solution across. panel edges using a method such as Lagrangian multipliers; the other is to form the functions so that they automatically provide continuous distributions. This second ineans was selected because of the inherent simplicity in the mathematical formulation. Figure 5 shows the basic tunction used for the new two-dimensional numerical scheme. This figure also implies that there is still a jump in the 


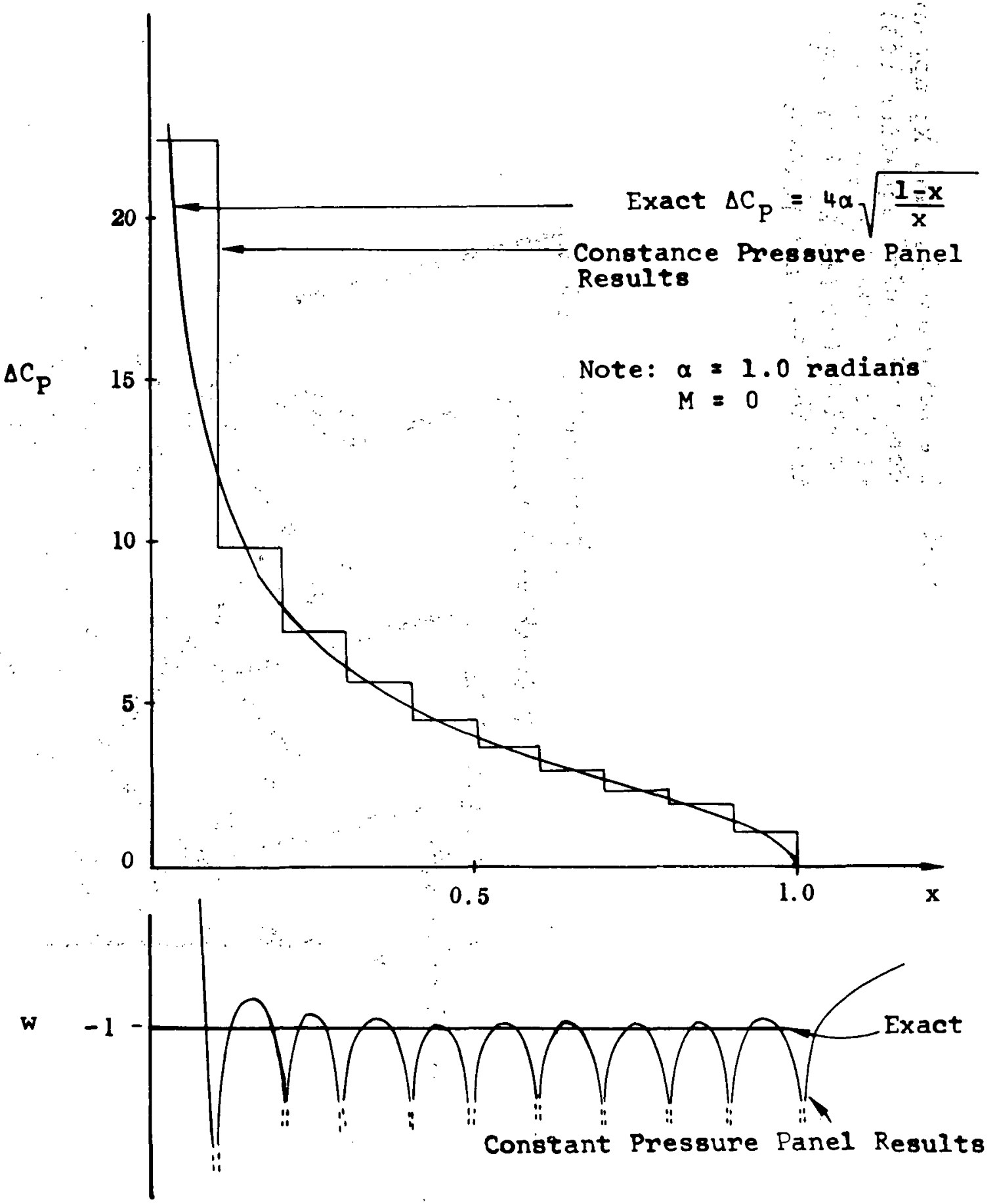

FIGURE 4 - TWO DIMENSIONAL CONSTANT PRESSURE PANEL RESULTS 

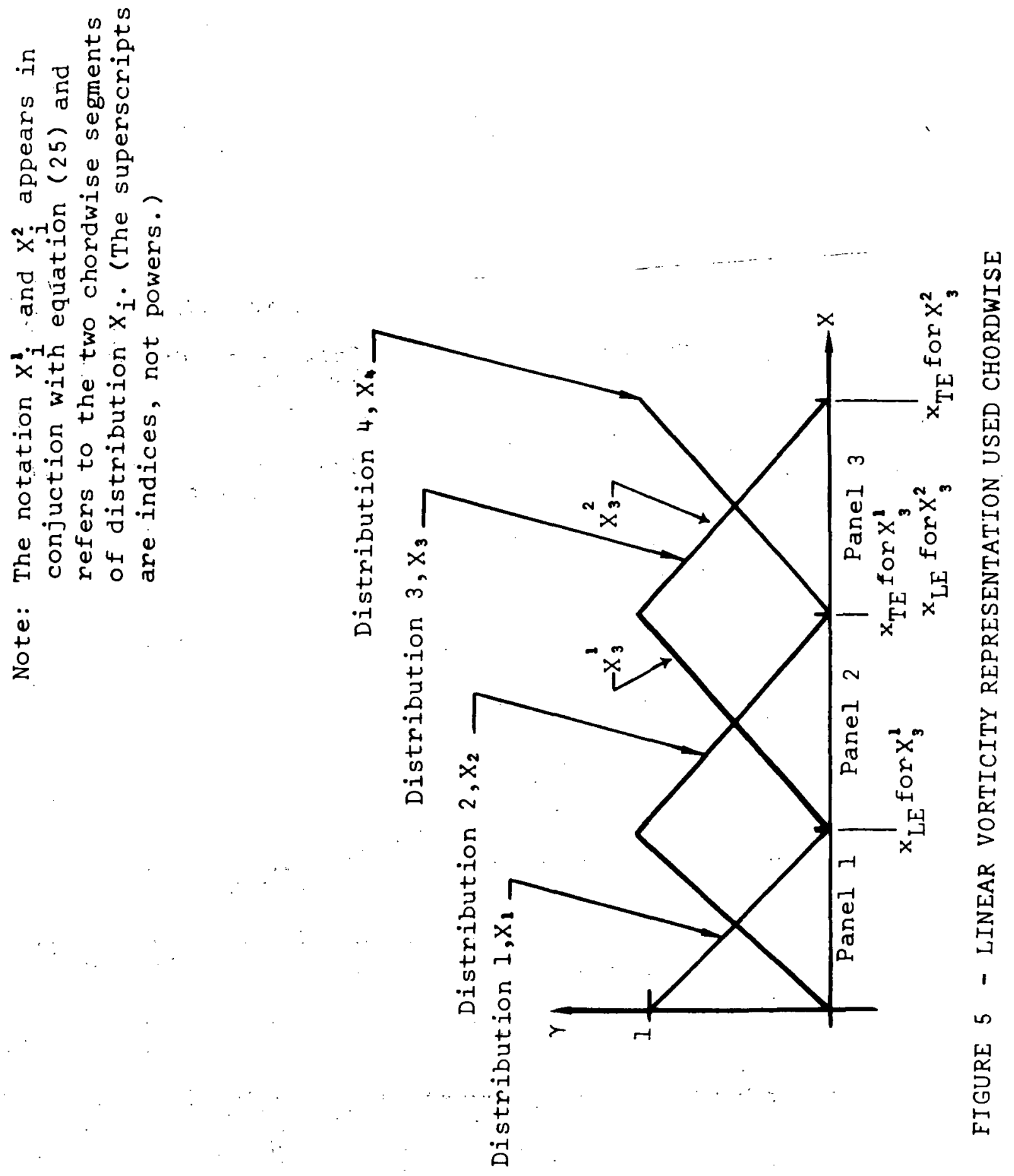
vorticity at the leading edge which causes a logarithmic singularity. The only way to romove this singularity at the leading edge is to use the proper asyoptctic form which is related to the reciprical of the square root of the distance from the edge (Figure 4). The new scheme was tested with and without the proper singular form, the conclusion was that there was sufficient accuracy for lift and moment without this singular variation in the vorticity.

Figure 5 snows that the triangular distribution of vorticity provides a natural means or introducing the kutta condition by omitting the last function. This omission forces the vorticity to vanish at the trailing edge.

The vortex spline formulaticn is based on a different means of satistying the boundary conditions which will be discussed later: but the fundamental approach is to compute the downvash at discrete locations using the basic triangular distributions of vorticity, $r$, in Equation (18) : with the limits changed to include two panels per distribution (Figure 5). Figure 6 shows the results for subsonic flow.

- Basic Three-Dimensional Function

For the three dimensional formulation of the scheme, two components of vorticity must be considered, $Y$ and $\sigma$. As pointed out parlier, these two components are not independent in potential flow. This means that it is not possible to arbitraily legislate that both components vary linearly in all directions. Using Equation (9) to solve for 0 in terms of $r$ gives:

$$
\sigma=-\frac{\partial}{\partial y} \int r d \ddot{x}
$$

From the two-dimensional study where $r$ was the only component present, the variation strearwise $(x)$ needed to avoid logarithmic singularities was linear. Equation (23) then states that o must vary quadratically in $x$. This same analysis can be repeated using the other requirement that $\sigma$ vary linearly normal to the stream (y) in order to eliminate singularities. Solving Equation (9), this time for $r$, yields:

$$
Y=-\frac{\partial}{\partial x} \int \sigma d y
$$

This means that $r$ must vary guadratically in $y$. 


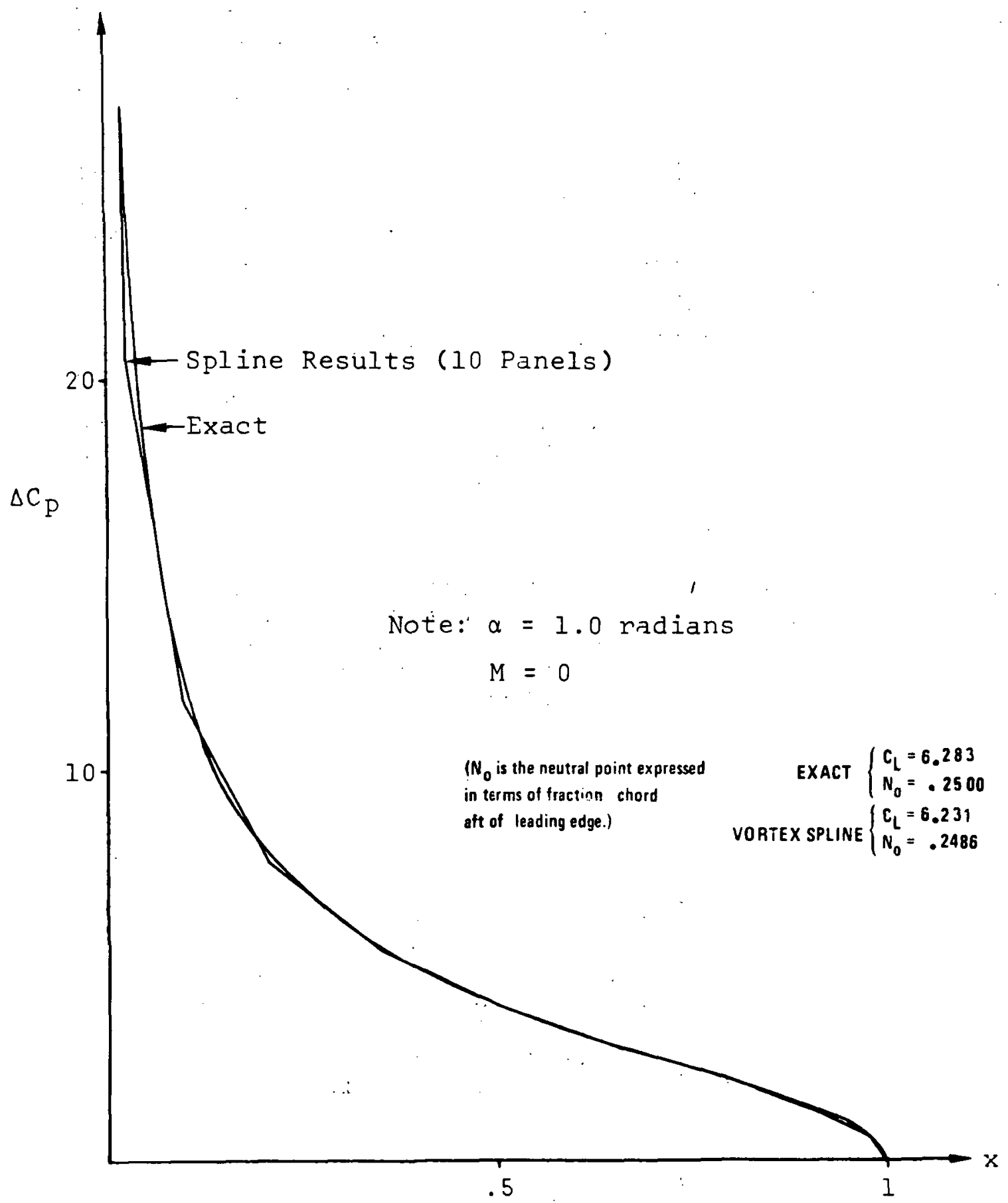

FIGURE 6 - TWO DIMENSIONAL SPLINE RESULTS 
Summarizing then:

$r$ - linear in $x$, quadratic in $y$

$\sigma$ - Iinear in $y$. quadratic in $x$

(A plot of o versus $y$ would be analogous to Figure 5a series of linear functicns.)

With this above variation the only places where singularities would occur on a simple rectangular wing would be at the leading edge (due to a jump in $r$ ) and at the tips (due to a jump in $\sigma$ ) for subsonic flow and at the tips cnly for supersonic flow (Figure 7).

Since the elementary horseshoe vortex is best described in terms of $r$, the criteria established above for this component of vorticity was the one used as the basis to derive the three dimensional distribution functicn presented next.

For the $x$ variation of $r$ in three-dimensions, the requirement shown above is the same as that for trodimensions. Hovever, the $y$ variation has to be quadratic or. in more fundamental terms, continuous in both value and slope in order for $\sigma$ to be continuous in value. The logical means to form such a surface distribution seemed to be as a product of two functions - one providing the desired linear $x$ variation and another providing the desired quadratic...y variation. To see how this y variation was formed, consider the basic triangular form used in two-dimensions. This distribution has, in general, three discontinuities in slope. By adding three discontinuous quadratic distributions to this basic form. these discontinuities can te removed. Figure 8 shows how this is accomplished. The requirement for each of the quadratic functions is that they begin and end at the centers of panels (spanwise) with zero value and slope, and also have a discontinuity in slope at the panel edge which cancels the discontinuity in slope of the triangular function. The overall function then becores six sefarate quadratics which cover four panels spanvise. The equations of these quadratics can be computed from the imposed boundary conditions cn each segment. Table 1 gives these results. (As a note, these spanwise functions could have also been developed using three quadratics over three panels specifying the slope and value matches at the two inner pant edges, and also requiring the distribution to vanish in both value and slope at the outside edges. The additional reguirement of unit maximus value would then completely specify the distribution.) 


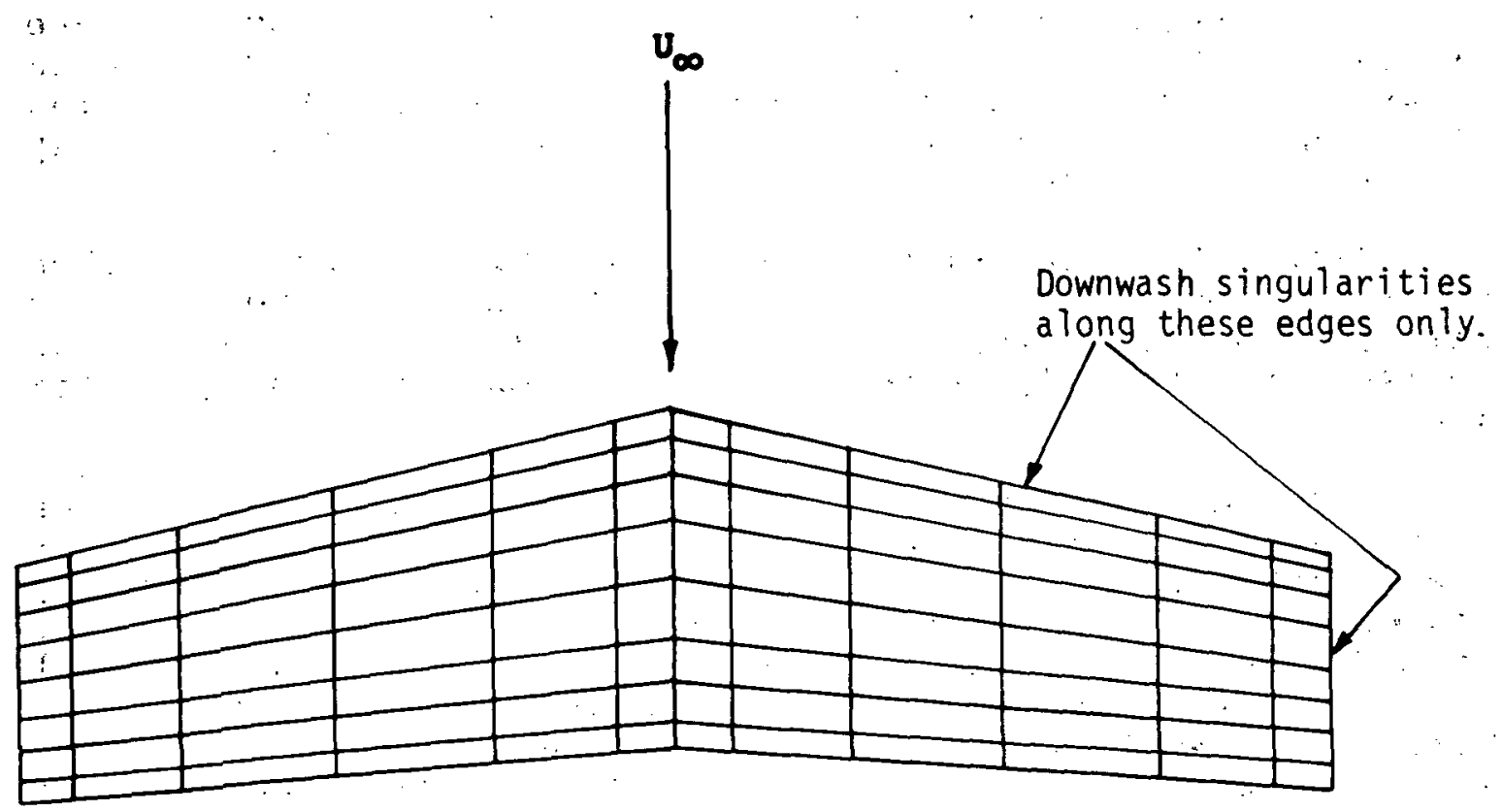

Note: no downwash singularity at leading edge, if edge is supersonic.

FIGURE 7 - LOCATION OF DOWNWASH SINGULARITIES WITH VORTEX SPLINE 


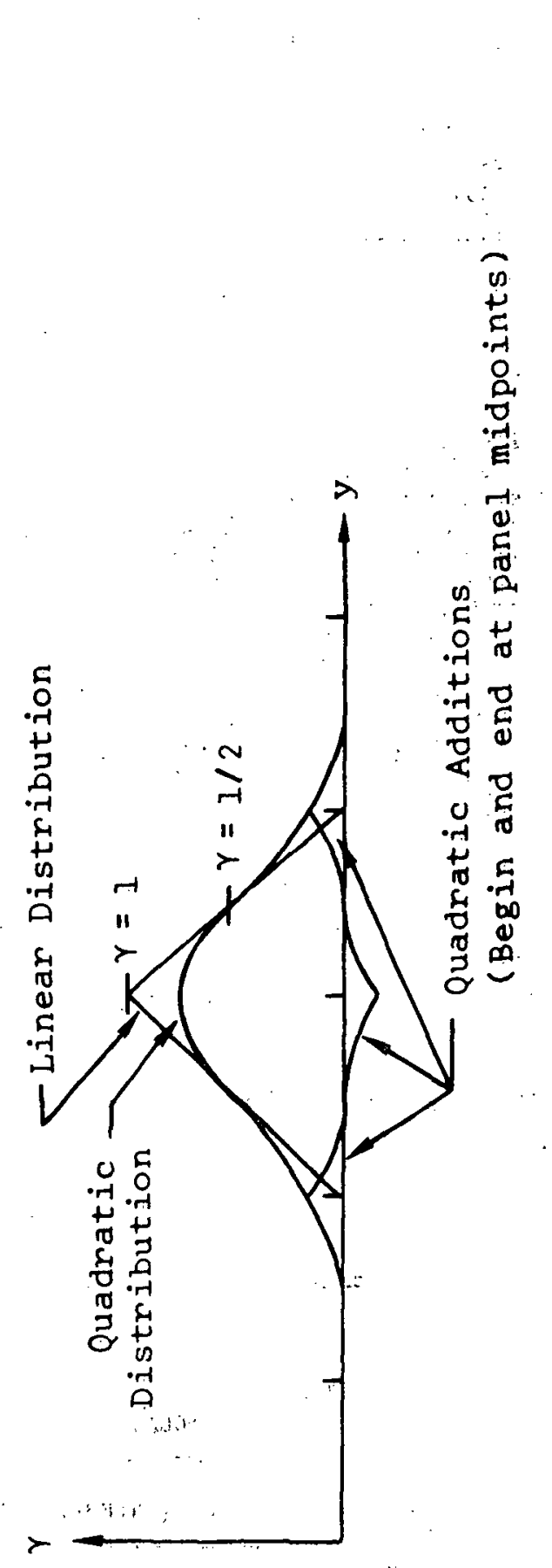




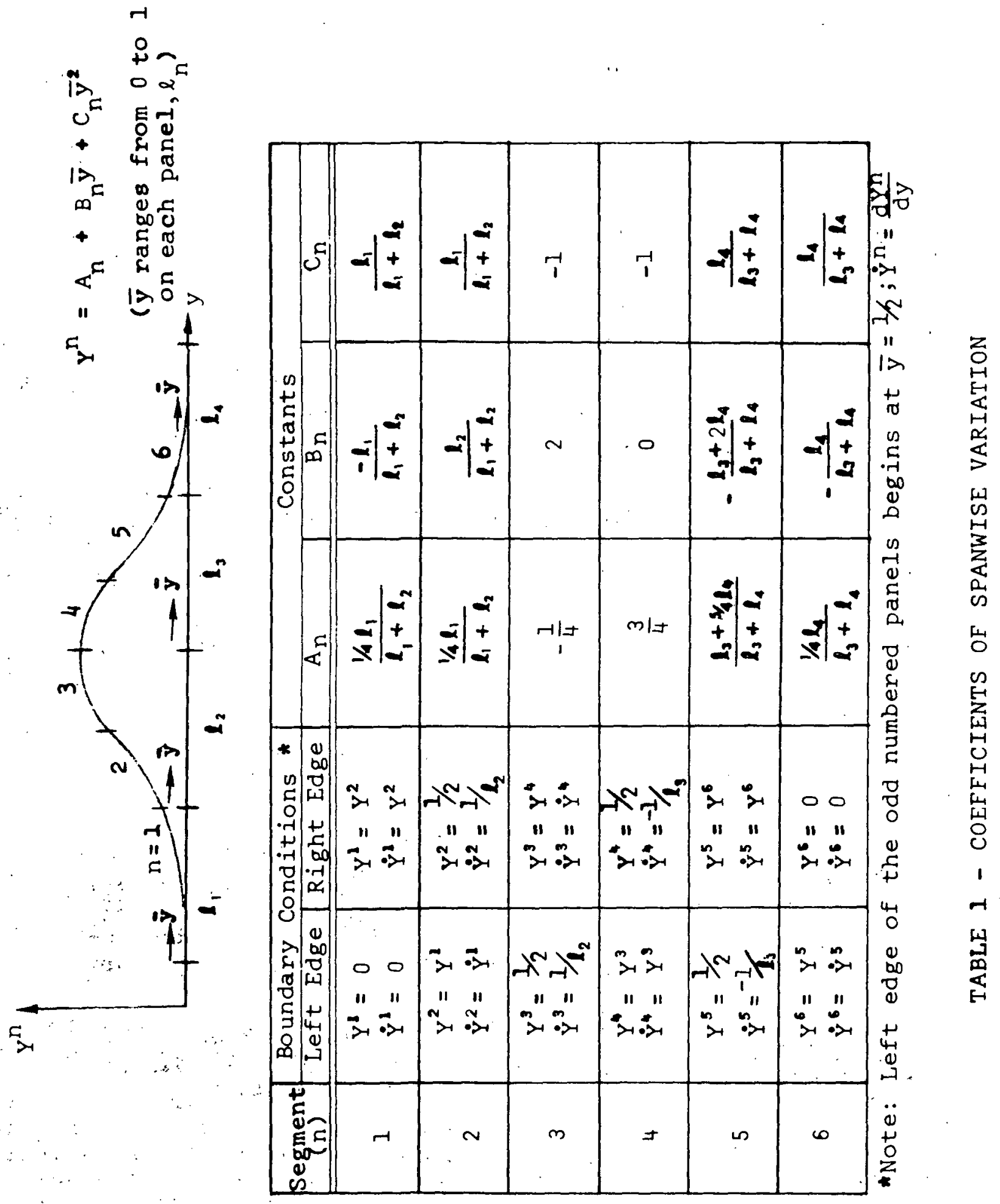


If $x_{1} i s$ used to represent the chordwise variation of $r_{k}$ and $y_{j}$ is used to represent the spanwise variation of $r_{k}$ the distribution of vorticity could be expressed as a doubly subscripted variable, $r_{1, j}$. Hovever, in order to conform with the singly. subscripted $r_{k}$ of Equation (14), a unique relationship between $k$ and $i, j$ was assigned so that:

$$
r_{k}=r_{1, j}=x_{1} Y_{j}: \quad k=1 \ldots N
$$

where $y_{j}$ consists of the six quadratic segments $Y_{j} \mathbf{n}$ (n = $1,2 \ldots .6)$ shown in $T a b l e 1$ and $x_{1}$ consists of the two linear segments $x_{1}^{m}(\pi=1,2)$ shcyn in Fiqure 5. Thus. the analytic description of $r_{k}=Y_{1, j}$ consists of twelve aiscrete parts, $X_{1}^{m} Y_{j}^{n}(m=1,2 ; n=1,2, \ldots, 6)$. These parts are distributed over eight panels (four spanwise, two chordwise) as shown in Figure 9 .

For a set of rectangular panels, $x_{1}$ is only a function cf the streanwise coordinate, $x$. By definition, y is only a

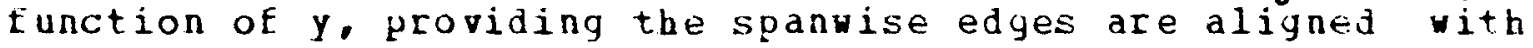
the free stream (which by the panel definitions, will always be the case). If the panels are not rectangular, $x_{1}$ becomes a function $D F$ coth $x$ and $y$ so that it varies as the fraction of local chord:

a nd

$$
x_{1}^{1}=\frac{x-x_{L E}}{x_{T E}-x_{L E}}
$$

$$
x_{1}^{2}=\frac{x_{T E}-x}{x_{T E}-x_{L E}}
$$

In the above equations. $x_{L E}$ and $x_{T E}$ are the leading apd trailing edge coordinates of the panel covered by segment $x_{1}$ $\left(\begin{array}{l}2 \\ 1\end{array}\right)$ : this is illustrated in Figure 5 .

ixcept for rectangular panels, $x_{1}^{1}$ and $x_{1}^{2}$ will be functions of $y$. If the fraction of local chora is defined as:

$$
\bar{x}=\frac{x-x_{L E}}{x_{T E}-x_{L E}}
$$

then $x_{1}$ becomes 


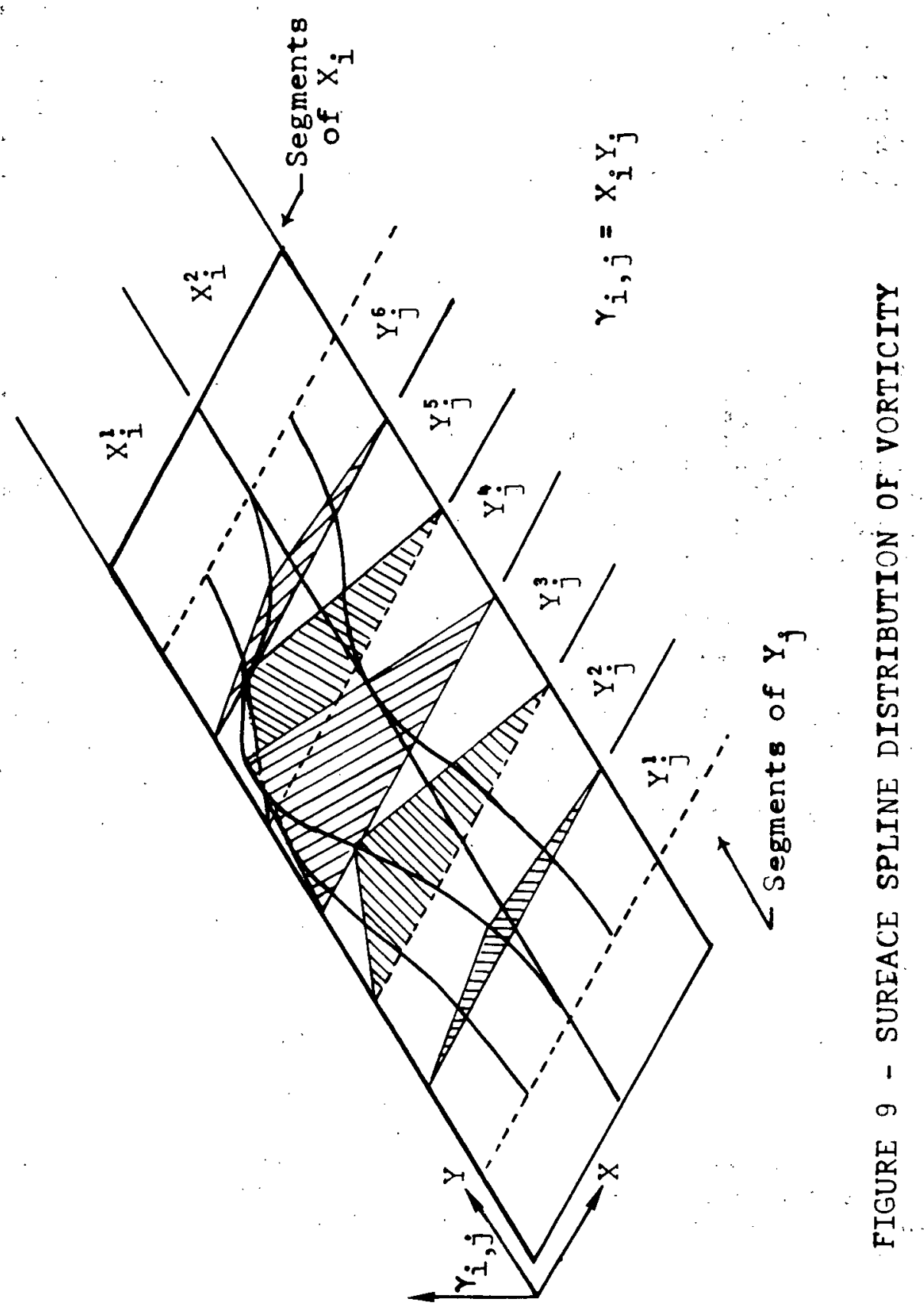




$$
x_{1}^{1}=\bar{x}
$$

a r.d

$$
x_{1}^{2}=1-\bar{x}
$$

The above representation holds for all leading and trailing, panel edye shapes.

The bound vorticity distribution defined by Equaticn (25) (termed spline) and shown in Figure 9; produces no singularities in the downush. This function is scaled up and down in the solution exactly the same way as the constant pressure panel scheme using a multiplicative constant which is the unknown.

The pext sections describe how a wirg is subdivided into panels and how the basic splines are overlapped using these subdivisions.

o Modeling Vorticity on a wing Using Vorticity Splines

Figure 10 shows a paneled wing with both a spanwise cut and a chordwise cut to display the chordwise and spanwise overlapping of the basic spline functions lassuming unit a mplitude). For the five chordwise functions and the six spanwise functions shown, there will be a total of thirty $(N=30)$ spline functions distributed over tuenty-five wing panels. The components of a single function are identified (shaded area) to depict its relationship to the overall arrangement. This figure also shows that the kutta condition has been imposed (zero value of furction at trailing edge).

As shown in Table 2, special functions are used at the root and tip, the functions for symetric flow will be discussed first.

The first root function (root function 1) is the right half (spanwise) of a normal function. The second root function (root function 2) is composed of segments $Y^{3}, Y^{4}, Y^{5}$ and $Y^{6}$ of a normal function ith $Y^{6}$ of the corresponding function on the left half of the wing added to $\mathrm{Y}^{2}$ of the right side function. Adding these two terms accounts for the overlapping of the right and left halves. (This can be done since the magnitude of functicn 


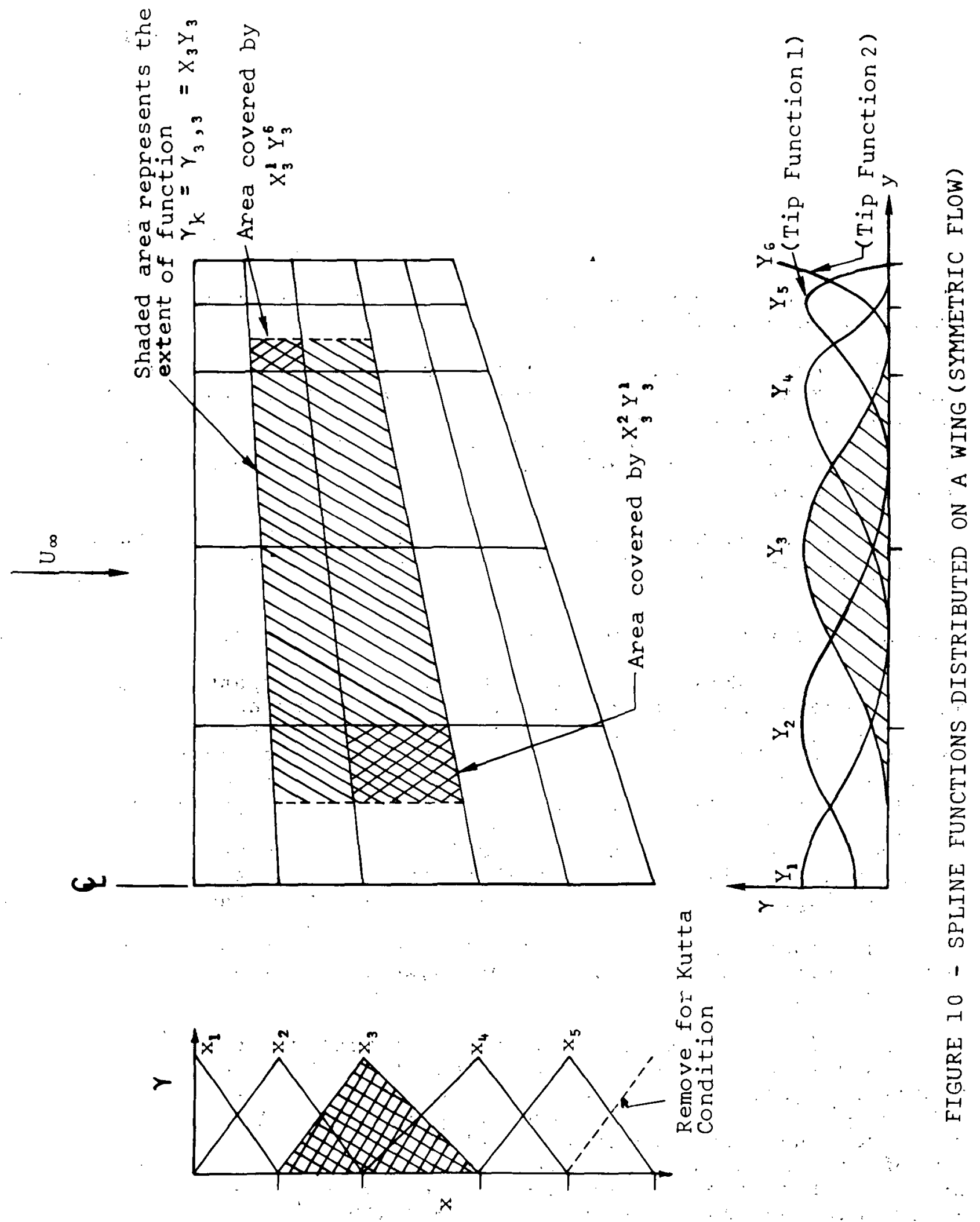


Root Function -- Symmetric

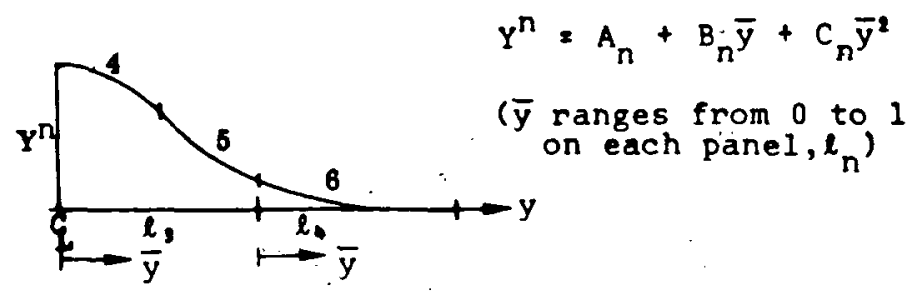

\begin{tabular}{|c|c|c|c|c|c|}
\hline \multirow{2}{*}{${ }^{8}{ }^{0}(n){ }^{t}$} & \multicolumn{2}{|c|}{ Doundary Condsticens } & \multicolumn{3}{|c|}{ Convetente } \\
\hline & Left nese & Peat Dose & $A_{n}$ & $\mathrm{z}_{\mathrm{n}}$ & $\mathbf{c}_{\mathrm{n}}$ \\
\hline $1-3$ & Do not & extst & - & $-\quad \therefore$ & $\therefore \quad-$ \\
\hline $4-6$ & Nornal & remetions & . & $\cdot$ & . \\
\hline
\end{tabular}

Note: The normal function represented by segments 4 through 6 at the root

meets the proper symmetry requitrement of vanishing slope.

Root Function 1 - Antisymmetric

Segments 1 through 6 do not exist

Root Function 2 - Symmetric

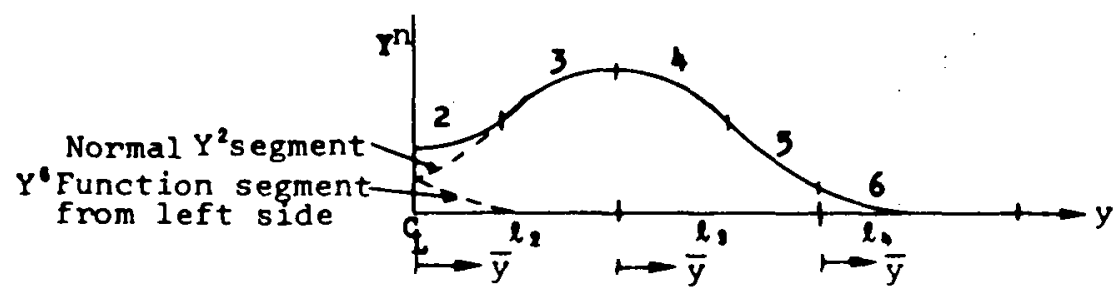

\begin{tabular}{|c|c|c|c|c|c|}
\hline \multirow{2}{*}{ (n) } & \multicolumn{2}{|c|}{ Downding Copdetions } & \multicolumn{3}{|c|}{ Conotionte } \\
\hline & Ioft Iepe & Edent Eso & $A_{n}$ & $\mathbf{B}_{n}$ & $c_{n}$ \\
\hline$i$ & Does nd & фt exist & - & - & - \\
\hline 2 & $\begin{array}{r}\text { Combine } \\
\text { functions }\end{array}$ & $\begin{array}{l}\text { over lapping } \\
\text { at the root }\end{array}$ & $1 / 4$ & 0 & 1 \\
\hline $3-6$ & Normal & functions & & & \\
\hline
\end{tabular}

TABLE 2 - SPECIAL SPANWISE FUNCTIONS 


\section{Root Function 2 - Antisymmetric}

Note: 2 \& are combined

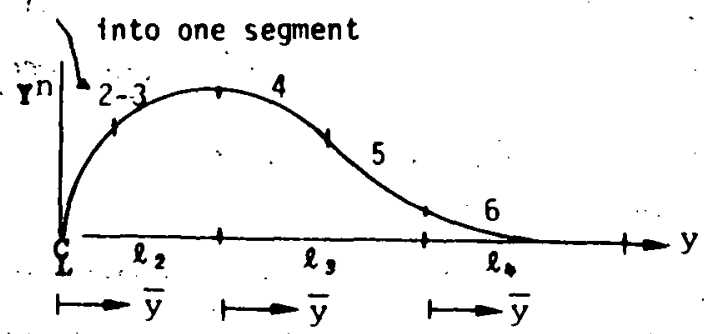

\begin{tabular}{|c|c|c|c|c|c|}
\hline \multirow{2}{*}{ Segment } & \multicolumn{2}{|c|}{ Bowndary Condttlous } & \multicolumn{3}{|c|}{ Conetente } \\
\hline & Left Eago & Faphe & $A_{n}$ & $\mathbf{B}_{\mathbf{n}}$ & $c_{n}$ \\
\hline $1: \ldots$ & Does. & not exist & - & - & - \\
\hline $2-3$ & $\mathrm{Y}^{2-3}=0$ & $\begin{array}{l}Y^{2-3}=Y^{*} \\
\dot{Y}^{2-3}=Y^{\circ}\end{array}$ & $\mathbf{0}$ & $3 / 2$ & $-3 / 4$ \\
\hline $4-6$ & Normal & functions & & & \\
\hline
\end{tabular}

Tip Function - 1

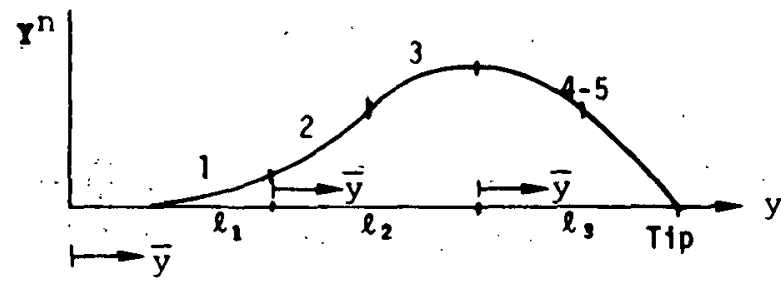

\begin{tabular}{|c|c|c|c|c|c|}
\hline & \multicolumn{2}{|c|}{ Doundary Coodstions } & \multicolumn{3}{|c|}{ Coantente } \\
\hline (n) & Left Bdge $:$ & Rdfet Bdo & $\boldsymbol{A}_{\mathrm{n}}$ & - Bn & $\therefore \mathbf{c}_{\mathbf{n}}$ \\
\hline . & Nomal: & functions & & . & $\overline{1 .}$ \\
\hline $4-5$ & $\begin{array}{l}Y^{4-5}=Y^{3} \\
\dot{Y}^{4-5}=\dot{Y}^{9}\end{array}$ & $Y^{-5}=0$ & $3 / 4$ & $\because 0 \quad \cdots$ & $-3 / 4$ \\
\hline 6. & does & not exist & - & - & - \\
\hline
\end{tabular}

TABLE 2 - CONTINUED 

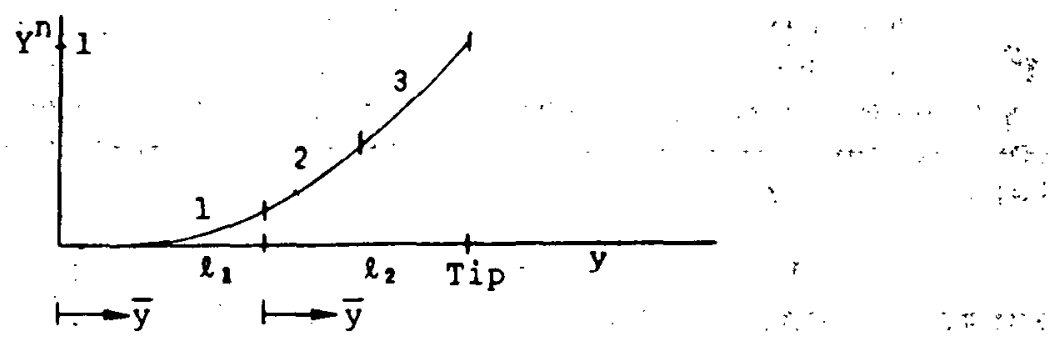

\begin{tabular}{|c|c|c|c|c|c|}
\hline \multirow{2}{*}{$\begin{array}{c}\text { segient } \\
(n)\end{array}$} & \multicolumn{2}{|c|}{ Bormdary Conditions } & \multicolumn{3}{|c|}{ Conotante } \\
\hline & Left Edeo & Boht Foso & $\mathbf{A}_{\mathbf{n}}$ & $B_{n}$ & $c_{n}$. \\
\hline 1 & Normal & function & & & \\
\hline $2 *$ & $\begin{array}{l}Y^{2}=Y^{1} \\
\dot{Y}^{2}=\dot{Y}^{3}\end{array}$ & $\begin{aligned} Y^{2} & =Y^{3} \\
\dot{Y}^{2} & =\dot{Y}^{3}\end{aligned}$ & $1 / 4 \frac{l_{1}}{l_{1}+l_{2}}$ & $\frac{l_{2}}{l_{1}+l_{2}}$ & $3 / 4 \frac{l_{1}-1 / 4 l_{2}}{l_{1}+l_{2}}$ \\
\hline 3 *. & $\begin{array}{l}Y^{3}=Y^{2} \\
\dot{Y}^{\prime}=\dot{Y}^{2}\end{array}$ & $\therefore$ & $1 / 4$ & 0 & $3 / 4$ \\
\hline $4-6$ & Do & not exist & - & - & - \\
\hline
\end{tabular}

- Note: An additional constraint was placed on segment 2 and 3 which required that the combined spanwise distribitions be capable of representing a constant

TABLE 2 - CONCLUDED 
corresponding to the $Y^{6}$ segment is the same as that corresponding to the $y^{2}$ segment-by symmetry. The reason for this treatment will be explained in the next section.)

The next to last tip function (tip function 1) has $Y^{l}$. $Y^{2}$. and $Y^{3}$ as normal segments. $Y^{6}$ does not exist: $y^{4}$ and $Y^{5}$ are one continuous quadratic function, the boundary conditions of which are: 1) continuous slope and value at left edge of the tip panel, and 2). zero value at the tip.

The last tip function (tip function 2) has $Y^{1}$ as a normal segment, but $y^{2}$ and $y^{3}$, the only remaining segments, are determined such that when this tip function is added to the other functions, the combination will produce a constant value of $r$ it all the strengths (multiplicative coefficients) are the same. This reguirement was set because, in supersonic flow, the vorticity., strength can be constant approaching a fointed tip. This tip distribution modeling capability also tended to yield the best numerical results of any of the variations studied (for both subsonic and supersonic flows).

Por antisymetric flow (e.g.. rolling) the roct functions have to be altered. Figure 11 shows these modified 'spanuise variations. The first root function $\left(Y_{2}\right)$ has been deleted. The most inboard function ( $\left.Y_{2}\right)$. for the antisymetric case, is the same as a retlection of the next to last tip function $\left(\mathrm{I}_{6}\right)$. The remaining functions across the span are the same as for the symetric case.

Table 2 gives all the coefficients for the special functions just described.

Rigure. 12 shous an example of hov two functions are overlapped - that is share common panels. This illustration depicts one of the many possible vays that overlapping can occur.

Arbitrary flow froblems involving symetric configurations can be decomposed into a symmetric and an antisymetric flow problea. In this way, only one half of the ving need be treated for each of these reduced problems. For symetric flow. the unknown strengths have the same values for corresponding functions on opposite sides of the configuration's plane of symmetry. For antisymetric flow, the strengths have the same magnitude but opposite sign for corresponding functions. Using these properties to formulate the problea results in the concept of real and image relations and the satisfaction of boundary conditions on one side of the plane of symetry only (the other side 


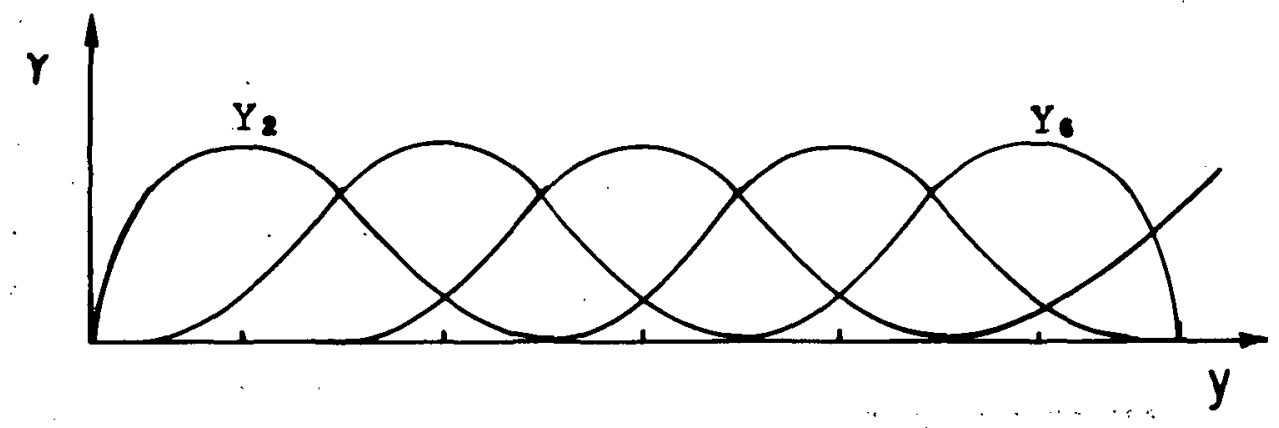

FIGURE 11 - ANTISYMMETRIC SPANWISE SPLINE DISTRIBUTION 


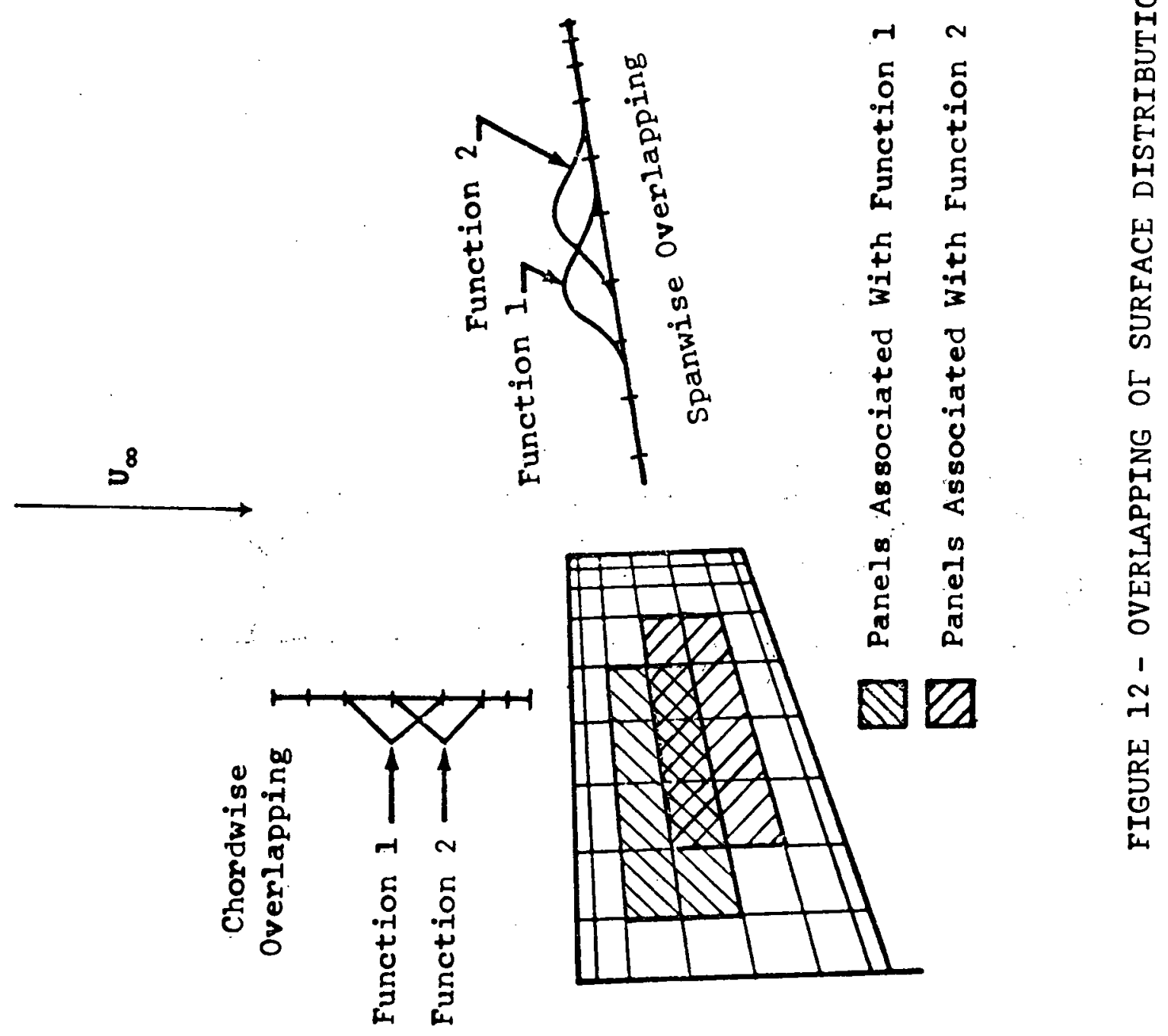


automatically satisfied by the appropriate symetry relation). The symetric problew is constructed as the sum of the influences from the real and image functions, where real and image implies corresponding functions on opposite sides of the symmetry plane. The antisymmetric problem is constructed as the difference of the influences from the real and image functions. This scheme reduces the number of calculations to be performed by half.

o wing Paneling

As mentioned earlier, the basic paneling procedure is to cut the wing streamise to form panels so that two panel edges are parallel to the free stream. This arrangement allows the kernel function integration to be performed more readily by introducing simple limits; it also simplifies the expression for the $Y$ variation of the basic vorticity distribution (making it a function of $y$ only).

The initial assumption for paneling was that the new technique use the same trapezoidal paneling as the constant pressure panel method (Ref. 1). Quickly. it was realized that any non-rectangular planforms would have discontinuities in the slopes of the fore and aft panel edges. Such discontinuities would introduce logarithmic singularities in the downash field, incluaing the wake. The source of these singularities can be seen by inserting the expression for the

$r$ spline distribution (Eq. 25 ) into Equation (23:).

$$
\sigma(x, y)=-\frac{\partial}{\partial y} \int_{x_{L E}(y)}^{x_{T E}(y), x} x(r, y) Y(y) d \zeta
$$

The upper limit of the above integral depends on whether o is computed on the distribution (Iinit equal $x$ ) or bebind the distribution (limit equal $x_{T E}(y)$ ).

The above equation shows that if any of the variables $I(y), x(x, y), x_{I E}(y)$ or $x_{I E}(y)$ has a discontinuity in slope with respect to y then o will be discontinuous in value producing the logarithmic singularity. of course $Y(y)$ is generated so that it is continuous in both value and slope so will not be of concern. This shows that planformibreaks, which cause jumps in panel: slopes. vill produce the undesirable down wash singularities.

A method yas developed to eliminate this proble by eliminating the planform breaks. Figure 13 shows the procedure and gives the resulting equations for the planfora edges. The procedure shown is exactly the same one used to 

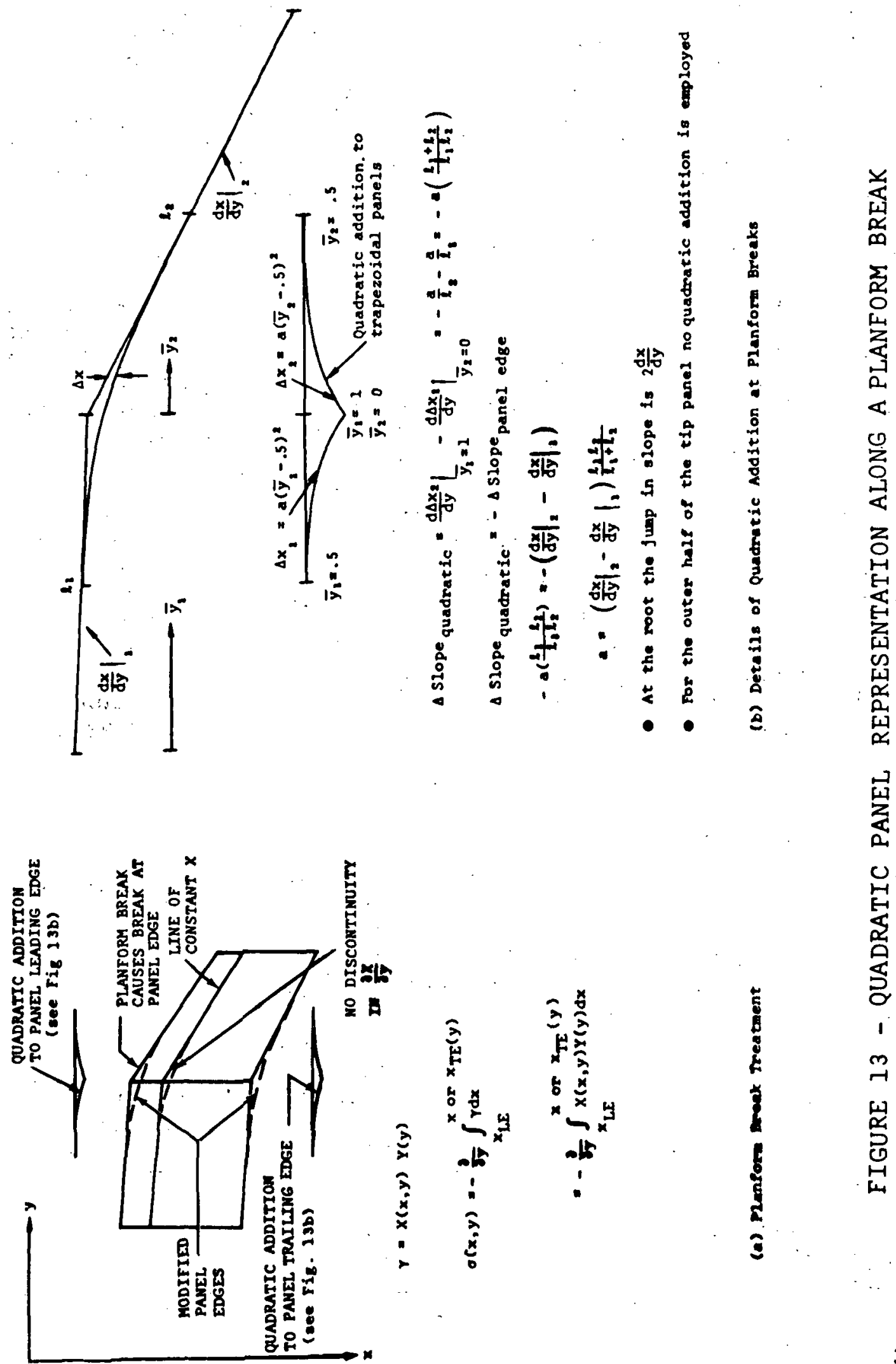
change the basic, linear distribution of vorticity into the quadratic distribution (Figure 8 ).

An additional benefit of going to the quadratic edge panels is that they provide a more general modeling element to handle arbitrary planforms. Later results in this report will show their usefulness in modeling a circular wing.

The basic panel then is one with two parallel, streamise edges with tro quadratic edges; the quadratic changes coefficients at mid sfan. with this paneling arrangement there will be no downash singularities in the wake (except from the tip)) and therefore, meets the desired requirement. (The quadratic paneling could also have been developed using one continuous quadratic across each panel edge: in order to he compatible ith the three spanvise fanel developments mentioned earlier.)

The paneling described above is the one always used in subsonic flow. For supersonic flow, however, there are tro choices of paneling - the one just described and another termed "speciai Mach line paneling." In this latter procedure certain Mach lines are identified, across which the vorticity level and/or gradient can te discontinuous (see Figure 1). These lines eminate from planform breaks, tips, etc. Along these lines continuity conditions can be relaxed and downstream sections can be treated as separate wings in the wake of the upstrean sections. Interior to each section, the paneling is treated as though that section vere a separate subsonic wing (normal quadratic panels), so that the $\gamma_{J}$ distributions for each subdivision do not overlap panels
of an adjacent subdivision.

Figure 14 shows a square wing at $M$ equal $\sqrt{2}$ comparing the normal geometric paneling with the special Mach line paneling. Note that the planform breaks are eliminated even wen they are due to the special Mach lines. Alsc note that since there are no sinqularities in the wakes emanating from each subdivision, the faneling of the subdivisions can be independent (except at plantorm breaks where the edges have to be aligned to ensure that the quadratic edges fore and aft coincide).

Numerical results computed with the special Mach line paneling (prfseated later in this report) show that this procedure greatly improves the details of the pressure distribution and at the same time allows for even greater variation in paneling options then arrorded by the normal geometric paneling. 


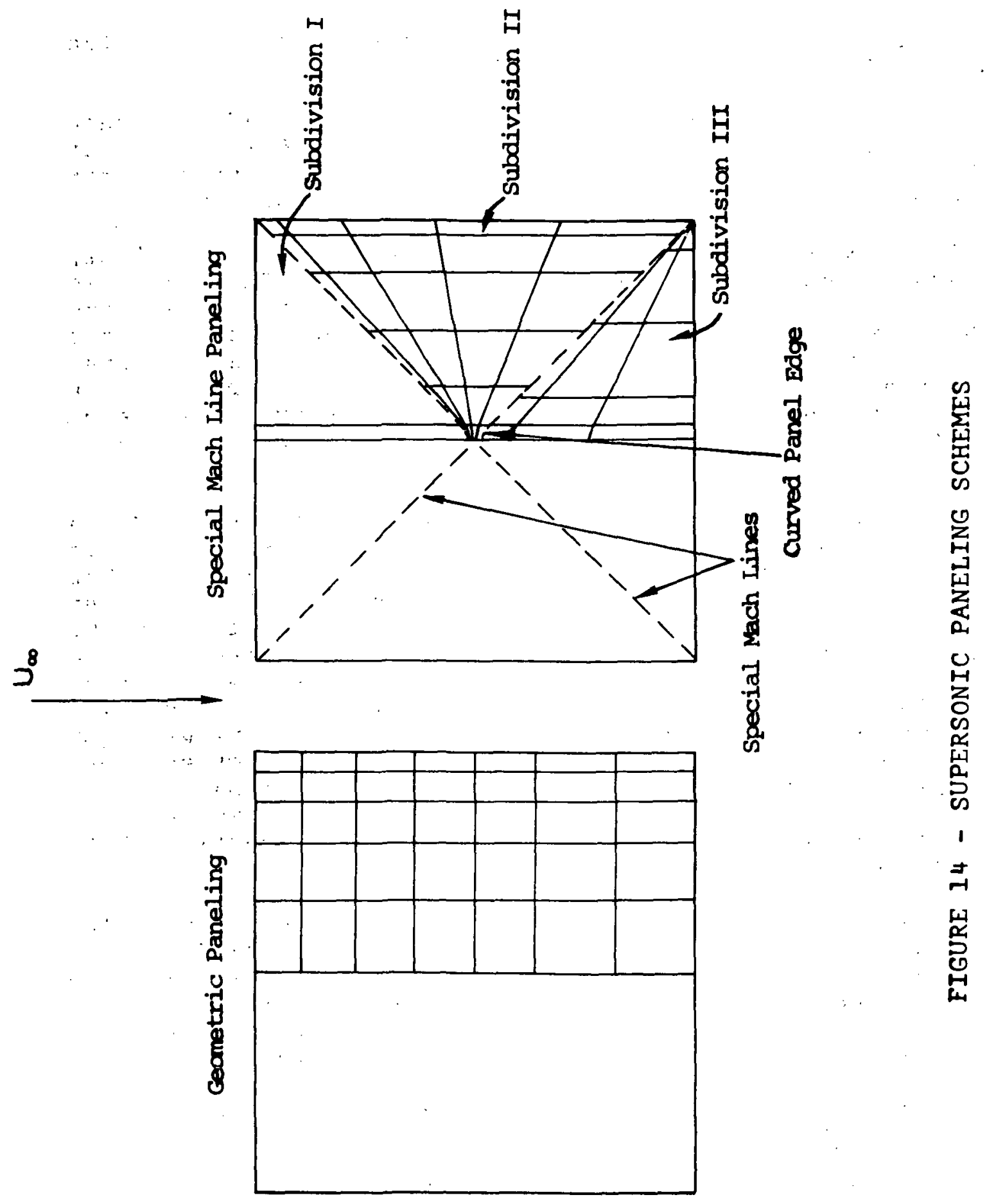


Equation (11) gave the integral expression wich relates the downush. w. to the vorticity distribution, $Y$. Equations (12) and (13) gave the kernel functions appearing in the integral for subsonic and supersonic flow. respectively.. This section discusses the evaluation of the integral for both flow regimes and the additional problem of establishing the limits for the supersonic integral.

Substituting the expression for $r_{j}, t_{\text {the }}$ basic spline distribution of vorticity (Equation (25), into the downwash integral. (Equation (11)) gives the downash at pcint $i$ due to unit amplitude of the jth spline distribution:

$$
w_{1 J}=\int_{y_{L}}^{y_{R}} \int_{x_{L E(n)}}^{x_{T E}(n)}[X(5 ; n) Y(n)]_{j} K\left(r-x_{1}, n-y_{1}\right) d s d n
$$

where [XY], denotes the right hand side of Equation (25).. I and $Y_{R}$ are the left and right edges, respectively, of the panel associated with $X_{j} y_{j}$ (components of [XY]). X $X_{T E}(n)$ and $X_{L E}(n)$ are the trailing and leading edges. respectively, of that same panel. The symbol frefers to the principle value of the integral (Ref. 5, pg. 132). The total downwash at $x_{1}$. $y_{1}$ is then the sum of the influences from all the integrals for the various spline distributions, each multiflied by its respective strength; $s_{j} \cdot w_{1 j}$ in Equation (32). corresponds to $A_{1 j}$ in Fquation (16):

$$
v_{1}-\sum_{j=1}^{N} A_{1 j} B_{j}
$$

Examination of the integral in Equation (32) shows that since $Y_{L}$ and $Y_{R}$ are constants, due to the fact that they are parallel edges. "the: integration can best be performed cn 6 first. Returning"to Equations (26) and (27) for $x$, it is noted that both can be written as:

$$
x= \pm \frac{s-f(n)}{c(n)}
$$


Were the variabies of integration, $\zeta$ and $n$, replace $x$ and $y$. respectively. Substituting Equation (33) into (32) and replacing A for wiJ yields two general forms:

$$
A_{1 J}^{1}= \pm \int_{y_{L}}^{y_{R}} Y(n) \frac{d n}{c(n)} \int_{x_{L E(n)}}^{x_{T E}(n)} r K\left(5-x_{1}, n-y_{1}\right) d \zeta
$$

and

$$
A_{1 J}^{2}=\mp \int_{y_{L}}^{y_{K}} Y(n) \frac{f(n)}{c(n)} d n \int_{x_{L E(n)}}^{x_{T E}(n)} K\left(\zeta-x_{1}, n-y_{1}\right) d \zeta
$$

For arbitrary edges the integrations indicated by Equations (34) and (35) cannot be performed analytically for both 6 and $n$. The integration in the 6 direction can be ferformed analytically, however, leaving the integration in the $n$ direction to be performed numerically. Even for the special cases when the integration can be performed entirely analgtically (e.g., rectangular panels). it is faster to perform the cuter, $n$ integration numerically. This is because of the number of hyperbolic functions, etc. Which arise from the analytical expression and wust be evaluated by the computer.

Letting:

$$
G_{1}(n)=\int_{x_{L E}(n)}^{x_{T E}(n)} \zeta K\left(\zeta-x_{1}, n-y_{1}\right) d \zeta
$$

and

$$
G_{2}(n)=\int_{x_{L E}(n)}^{x_{T E}(n)} K\left(\zeta-x_{1} \cdot n-y_{1}\right) d \zeta
$$

the resulting expressions for $G(n)$ in subsonic flow can be uritten a s:.

$$
\begin{aligned}
G_{1}(n)= & \frac{1}{4 \pi} \sqrt{\left.y_{1}-n\right)^{2}}\left[\frac{\zeta^{2}}{2}-\frac{\left(x_{1}+\zeta\right)}{2} \sqrt{\left(x_{1}-\zeta\right)^{2}+\theta^{2}\left(y_{1}-n\right)^{2}}\right] \\
& -\frac{\beta^{2}}{8 \pi} \ln \left|\left(x_{1}-\zeta\right)+\sqrt{\left(x_{1}-\zeta\right)^{2}+B^{2}\left(y_{1}-n\right)^{2}}\right|_{\zeta=x_{L E}(n)}^{\zeta x_{T E}(n)} \\
G_{2}(n)= & \frac{1}{4 \pi} \frac{1}{\left(y_{1}-n\right)^{2}}\left[\left(-\sqrt{\left(x_{1}-\zeta\right)^{2}+\theta^{2}\left(y_{1}-n\right)^{2}}\right]_{\zeta=x_{L E}(n)}^{\zeta=x_{T E}(n)}\right.
\end{aligned}
$$


and in supersonic flow as:

$$
\begin{aligned}
& G_{1}(n)=-\frac{1}{2 \pi}\left[\frac{x_{1}+\zeta}{2\left(y_{1}-n\right)^{2}} \sqrt{\left(x_{1}-\zeta\right)^{2}-B^{2}\left(y_{1}-n\right)^{2}}\right.
\end{aligned}
$$

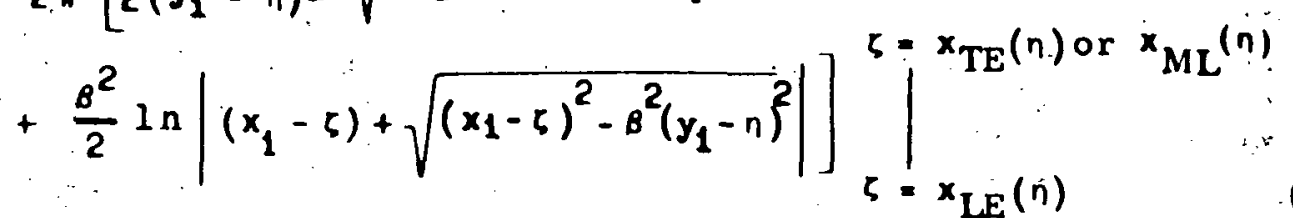

$$
\begin{aligned}
& \zeta=x_{\operatorname{LE}}(\hat{n}) \\
& G_{2}(n)=-\left.\frac{1}{2 \pi} \frac{1}{\left(y_{1}-n\right)^{2}} \sqrt{\left(x_{1}-\zeta\right)^{2}-B^{2}\left(y_{1}-n\right)^{2}}\right|_{\zeta=x_{L E}(n)} ^{\zeta=x_{T E}(n)}
\end{aligned}
$$

The notation at the right side of the above expressions is the standard one for integrals, indicating that the expression is first evaluated with $\zeta$ replaced by the upper linit and then. subtracted from that, the expression with $\zeta$ replaced by the lover limit.

Under certain circumstances Equations (38) through (41) can become singular due to the $\left(y_{1}-n\right)^{2} t \in[m$ in the denominator. The order and presence of a singularity in the integrand depend on the relationship of $y_{\text {f }}$ to the two side edges of a panel. $Y_{L}$ and $y_{R}$. and the relationshif of $x_{1}$ to the other two edges $x_{\text {LE }}(n)$ and $x_{T E}(n)$. It is obvious that if the control point y value falls to the left of $Y_{L}$ ( $t$ he left edge) or to the right of $Y_{R}$ (the right edge) the integrals involving $\left(y_{1}^{-n)}\right.$ yill never be singular. For this case, the numerical integration with respect to $n$ from $Y_{L}$ to $Y_{R}$ can be cerformed routinely using Gaussian quadrature. It is also true, but less obvious, that if the $x$ value of the contrcl pcint, $x_{1}$. lies upstream of $x_{L E}\left(Y_{1}\right.$ ) then there will be no singularity regardless of the $y_{1}$ value (this can be verified by taking the limit of the expression evaluated at both limits as $n$ approaches $y_{1}$ l. For this last case, however, care wust be taken that none of the quadrature points falls too close to $y_{1}$, when $y_{1}$ falls between $y_{L}$ and $y_{R}$. Such proximity could give rise tc numerical problems. Other than taking this precautionary measure, the integration is routine.

If the $y_{1}$ value falls between $y_{L}$ and $y_{R}$ and the $x_{1}$ value is downstream of the leading edge, $x_{L E}\left(Y_{1}\right)$, then the $G(n)$ functions cannot be integrated directly. For this case there will be three basic singular forms: 1) a logarithmic singularity (for only the linearly varying terms, $\left.\left.G_{1}\right), 2\right)$ a Cauchy singularity(1/y,-nfcris). and 3) a Mangler singularity $\left(1 /\left(y_{1}-n\right)^{2}\right.$ form). The first singularity is integrable in a Riemanian sense, but the latter two are not. These singular forms arise from taking the liniting for of the kernel function as the point of evaluation approaches the 
plane of the singularity. There would be no singularities if the limiting process had been taken after the integration. The advantage of taking this linit before integrating is the resulting simpler expression. The cost of taking this linit is the introduction of the singular forms. Although the Mangler and Cauchy expressions cannot be numerically integrated, they can be eliminated from the integrand and treated separately (Ref. 7). There are several ways to accomplish this, but basically all the methods assume that the integrand can be expanded about the singularity as:

$\bar{G}(n)=\frac{A_{M}}{\left(y_{1}-n\right)^{2}}+\frac{A_{C}}{\left(y_{1}-n\right)}+A_{L}+n\left|\left(y_{1}-n\right)\right|+A_{0}+A_{1}\left(y_{1}-n\right)+A_{2}\left(y_{1}-n\right)^{2}+\ldots$

(42)

Here $\bar{G}(n)$ represents either $G_{1}(n)$ or $G_{2}$ (n) multiplied by the remaining $n$, functionality (from Fquations (34) and (35)).

Fixpansion 42 can be performed either analytically or nunerically. The analytical nethod is covered in Reference 7 . so it ill not be repeated here.

Nuerically $\bar{G}(n)$ can be expressed as:

$$
\bar{G}(n)=\bar{G}_{S}(n)+\bar{G}_{R}(n)
$$

were $\bar{G}_{8}(\eta)$ contains all the singular forms and $\overline{\mathbf{G}}_{\mathbf{R}}(n)$ is regular. In the vicinity of the singularity. the leading terms of the series in Equation (42) dowinate, therefore:

$$
\bar{G}(n): \cong \frac{A_{M}}{\left(y_{1}-n\right)^{2}}+\frac{A_{C}}{y_{1}-n}+A_{L} 1 n\left|y_{1}-n\right|+A_{0}
$$

\section{(In region of singularity)}

The only tera of the above series uhich is antisymetric about the singularity is the cauchy tern, Ac/ $y_{1}-n$. therefore, it is possible to evaluate the coefficient of this term independently by computing $\bar{G}(n)$ at two points clcse to the singularity ${ }^{-i}=y_{1}+\varepsilon_{1}$ and $n=y_{1}-\varepsilon_{1}$ :

$$
A_{c} \cong \frac{\varepsilon_{1}}{2}\left[\bar{G}\left(y_{1}-\varepsilon_{1}\right)-\bar{G}\left(y_{1}+\varepsilon_{1}\right)\right]
$$

The choice of $E_{2}$ is a function of the number of significant Eigures the nachine uses to store numbers and, to a lesser extent, 
the manner in which the expression for $\bar{G}(n)$ is constructed. (The accuracy of $\bar{G}(n)$ near the singularity is best if the origin of the axis system is located at the singularity. This origin location aroids the numerical prcblem of adding a small number to a large one.)

Since the remaining terms of Equation (44) are all symetric about the singularity, they must be evaluated simultaneously. The three unknouns $A_{M}, A_{L}$. and $A_{0}$ can be numerically computed by evaluating $\bar{G}(n)$ at three points. Because the Cauchy term has a rather large influence, numerical error can be reduced by eliminating this antisymmetric term (as well as the other antispmetric terms) from the calculation by evaluating $\bar{G}(n)$ at equal distances on both sides cf the singularity and suming the influences. This gives three equations for the three unknowns:

$$
\begin{aligned}
& \frac{2}{\varepsilon_{1}^{2}} A_{M}+2 \ln \left|\varepsilon_{1}\right| A_{L}+2 A_{0}=\bar{G}\left(y_{1}+\varepsilon_{1}\right)+\bar{G}\left(y_{1}-\varepsilon_{1}\right) \\
& \frac{2}{\varepsilon_{2}^{2}} A_{M}+2 \ln \left|\varepsilon_{2}\right| A_{L}+2 A_{0}=\bar{G}\left(y_{1}+\varepsilon_{2}\right)+\bar{G}\left(y_{1}-\varepsilon_{2}\right) \\
& \frac{2}{\varepsilon_{3}^{2}} A_{M}+2 \ln \left|\varepsilon_{3}\right| A_{L}+2 A_{0}=\bar{G}\left(y_{1}+\varepsilon_{3}\right)+\bar{G}\left(y_{1}-\varepsilon_{3}\right)
\end{aligned}
$$

These equations can be solved for ${ }^{A_{M}}$ and ${ }^{A_{L}} \cdot{ }^{A_{0}}$ will nct be required as will be shown next.

Using the above results, $\bar{G}_{S}(n)$, Equation (43), can be expressed as:

$$
\bar{G}_{g}(n)=\frac{A_{M}}{\left(y_{1}-n\right)^{2}}+\frac{A_{c}}{y_{1}-n}+A_{L} \ln \left|y_{1}-n\right|
$$

The above expression can be integrated directly using the integrals given for the Mangler and Cauchy forms in Reference 7.

$$
\int_{y_{L}}^{y_{R}} \frac{A_{M}}{\left(y_{1}-n\right)^{2}} d n \equiv A_{M}\left[\frac{1}{\left(y_{1}-y_{R}\right)}-\frac{1}{\left(y_{1}-y_{L}\right)}\right]
$$




$$
\begin{gathered}
\int_{y_{L}}^{y_{R}} \frac{A_{C}}{y-n} d n \equiv A_{C}\left[-\ln \left|\frac{y_{1}-y_{R}}{y_{1}-y_{L}}\right|\right] \\
\int_{y_{L}}^{y_{R}} A_{L} \ln \left|y_{1}-n\right| d n=A_{L}\left[-\left(y_{1}-y_{R}\right) \ln \left|y_{1}-y_{R}\right|\right. \\
\left.+\left(y_{1}-y_{L}\right) \ln \left|y_{1}-y_{L}\right|-y_{R}+y_{L}\right]
\end{gathered}
$$

The non-singular part of $\bar{G}(n)$ is then:

$$
\overline{\boldsymbol{\sigma}}_{R}(n)=\bar{G}(n)-\bar{G}_{S}(n)=\bar{G}(n)-\frac{A_{M}}{\left(y_{1}-n\right)^{2}}-\frac{A_{C}}{y_{1}-n}-A_{L}|n| y_{1}-n \mid
$$

The above expression picks uf the $A_{0}$ and remaining terms which vere dropped to compute the ${ }_{M}, A_{C}$, and $A_{L} \cdot \bar{G}_{R}(n)$ contains no forms which cannot be integrated numerically using Gaussian quadrature. Therefore, numerical integraticn of $\bar{G}_{R}(n)$, when combined with the results shcwn in Equations (48) through (50), constitute the entire integration of $\bar{G}(n)$ to evaluate $A_{1 j}$. (It is important to mention again that the allovable magnitudes for $c_{1}, c_{2}$, and $c_{3}$ depend on the number of significant figures carried for $G(n)$ and are therefore computing machine defendent.)

The above procedure was compared to a method which used the non-singular, out of plane expression for the kernel. This alternate procedure integrates the out of plane kernel using a control point very close to the singularity plane. For the same accuracy, the planar conputational scheme, just described, was. about ten times faster. Both schemes used a self checking integrator which breaks the interval of integration intc farts small enough to be handled by a four point Gaussian quadrature using eight points as a check (Reference 8 ).

The 5 integration in supersonic flow is not always between the leading and trailing edges. Figure 15 shows the forward Mach lines emanating from the point $x_{1}, y_{1}$. These lines are shown cutting a panel, the significance of this cut is that portions of the panel ahead of the lines influence the point $x_{1} y_{1}$. yhile those aft do not. In terms of the kernel function, the regions aft of the lines cause the values in the radicals to become negative maxing the expression for the integrand conplex. Physically, this means that the influencing region is limited to the area vithin the forward Mach lines, therefore, the limits of integration should be adjusted to include only this region. Pigure 15 shows two distinct regions of integration, one from the panel leading edges to the panel trailing edges - Region I: and one from the panel leading edges to the Mach lines - Region II. The integration over a panel is the sum of the integrals over the 
i.

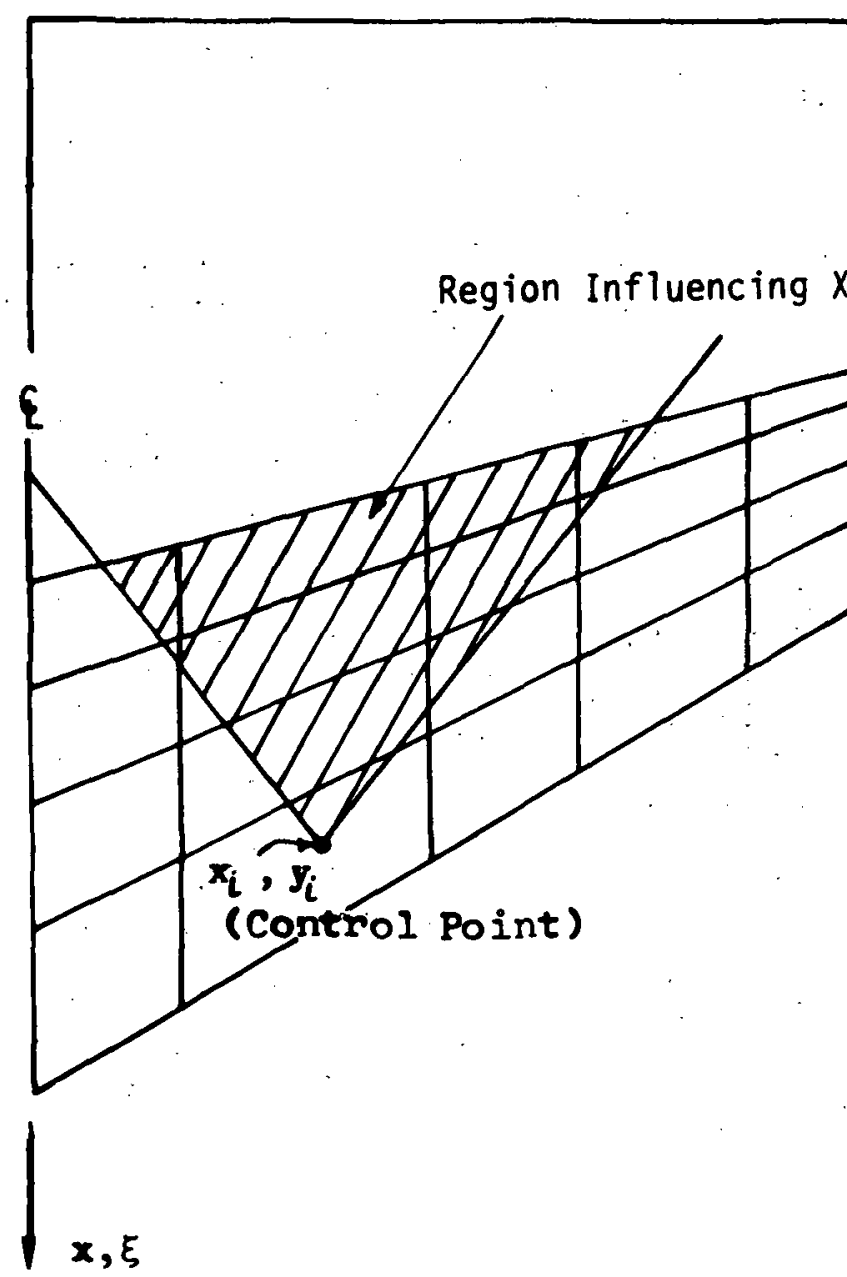

$\mathbf{y}, n$

FIGURE 15 - FORWARD MACH CONE ON SURFACE OF A WING 
two types of regions (as will be shown in figure 16, there can te several of each type on a single panell.

The integration of the supersonic kernel in the 6 direction from leading edge to trailing edge was given in Equations (40) and (41). The integral frow leading edge to Mach line is the same except for the upper limit, $X_{T E}(n)$. replaced by the equation of the forward Mach line:

$$
\begin{aligned}
\zeta_{M L}=x_{1} \mp \beta\left(n-y_{1}\right) & - \text { right going Mach line } \\
& + \text { left going Mach line }
\end{aligned}
$$

This upper limit on 6 simplifies the expressions in Equations (40) and (41) by forcing the radicals to vanish. The integrands for the $n$ integration wich result from this changing of limits on $r$ are therefore no more compicated to integrate than the cnes already discussed: The problem then is identifying the various regions on the panel.so. that the limits for the $n$ integrations can be determined.

For curved panel edges, a Mach line can intersect in various ways. Figure 16 shors some of these combinations. Several intersection characteristics can be noted from the figure:

1. After an intersection vith a trailingedge; the Mach line can intersect either the trailing edge again or the leading edge.

2. After an intersection with a leading edge the Mach line can intersect either the leading edge again or the trailing edge.

3. Proceeding left to right (positive $n$ or y direction) each intersection changes the region - from a Region I to a Region II from a Region II to a Region I and from a Region II to a Region 0 (Region 0 does not influence the contrcl foint): but. there can never he a jump from a Region $I$ to a Fegion 0 or vice versa.

The above characteristics can be used to form a logical scheme to identify the various regions and find the limits of integration. Using the quadratic equation for the planform edges from Figure 13 and the equation for the Mach lines (52) expressed in a comon coordinate system, a quadratic expression for the intersections can te obtained:

$$
x_{1} \mp B\left(n-y_{1}\right)-a-b n-c n^{2}=0
$$

where $a+b n+c n^{2}$ is the squation of the panel edge. 

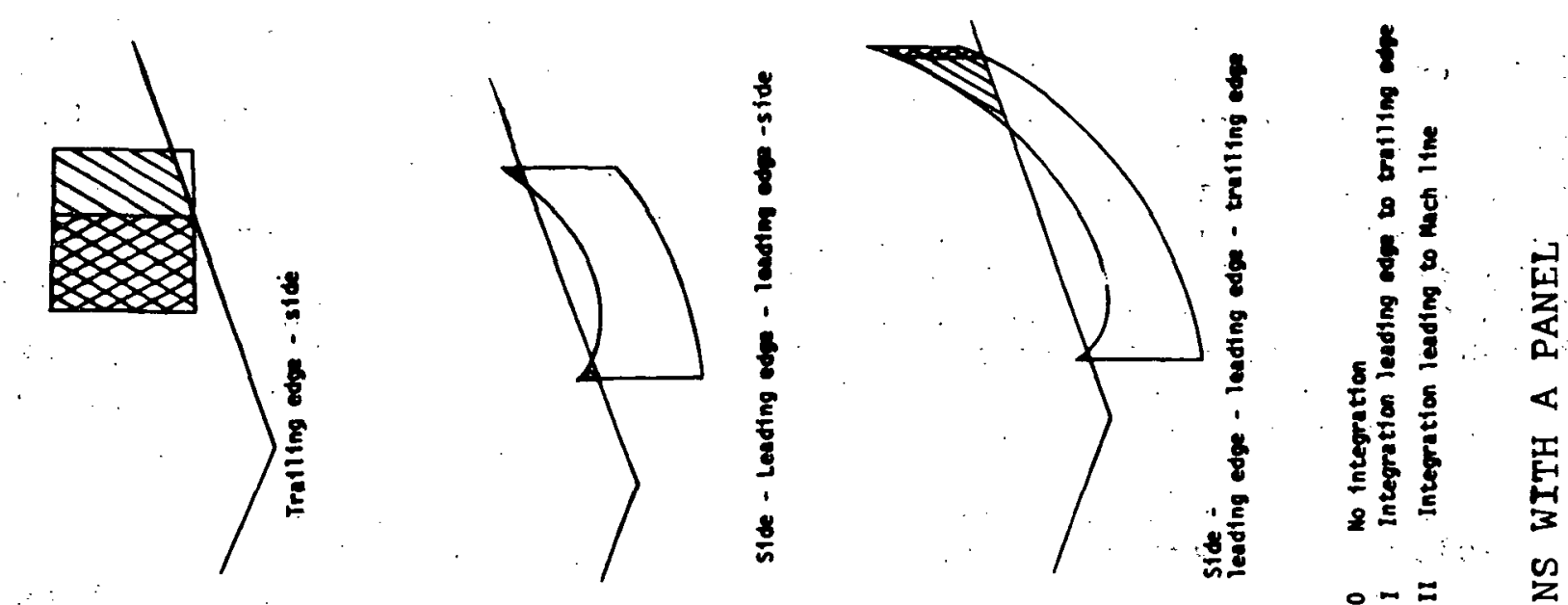

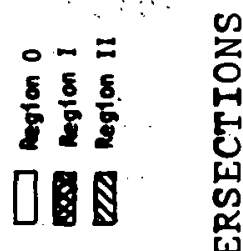
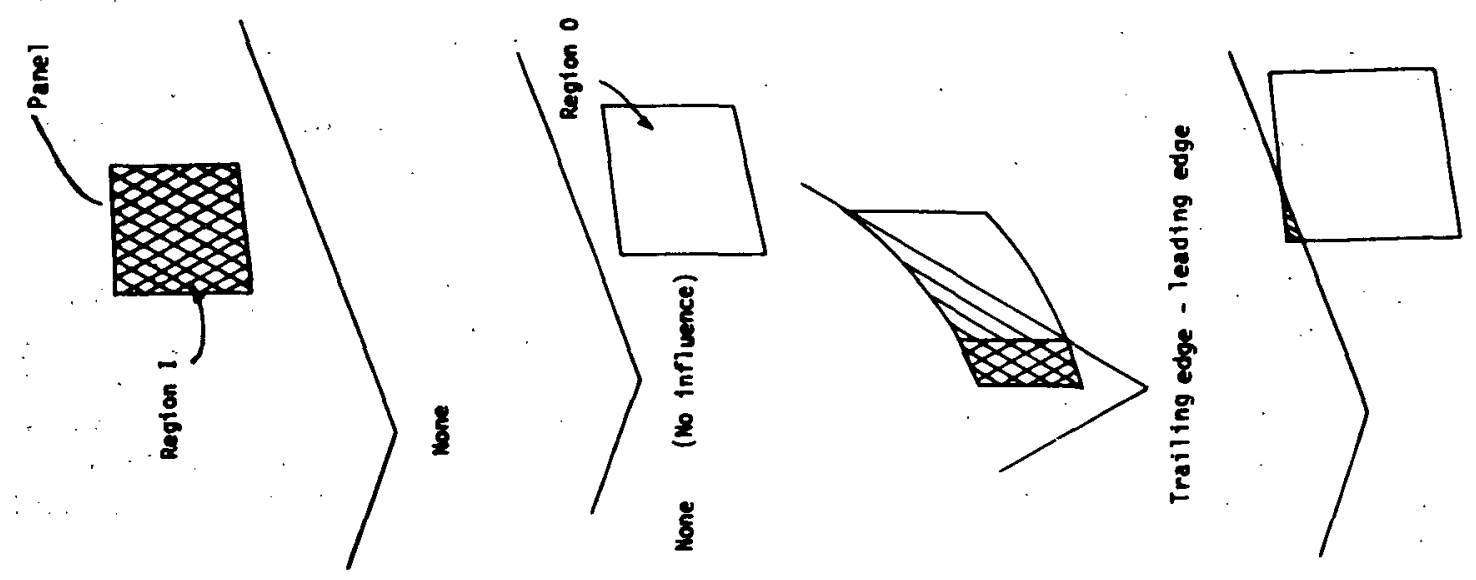

垈
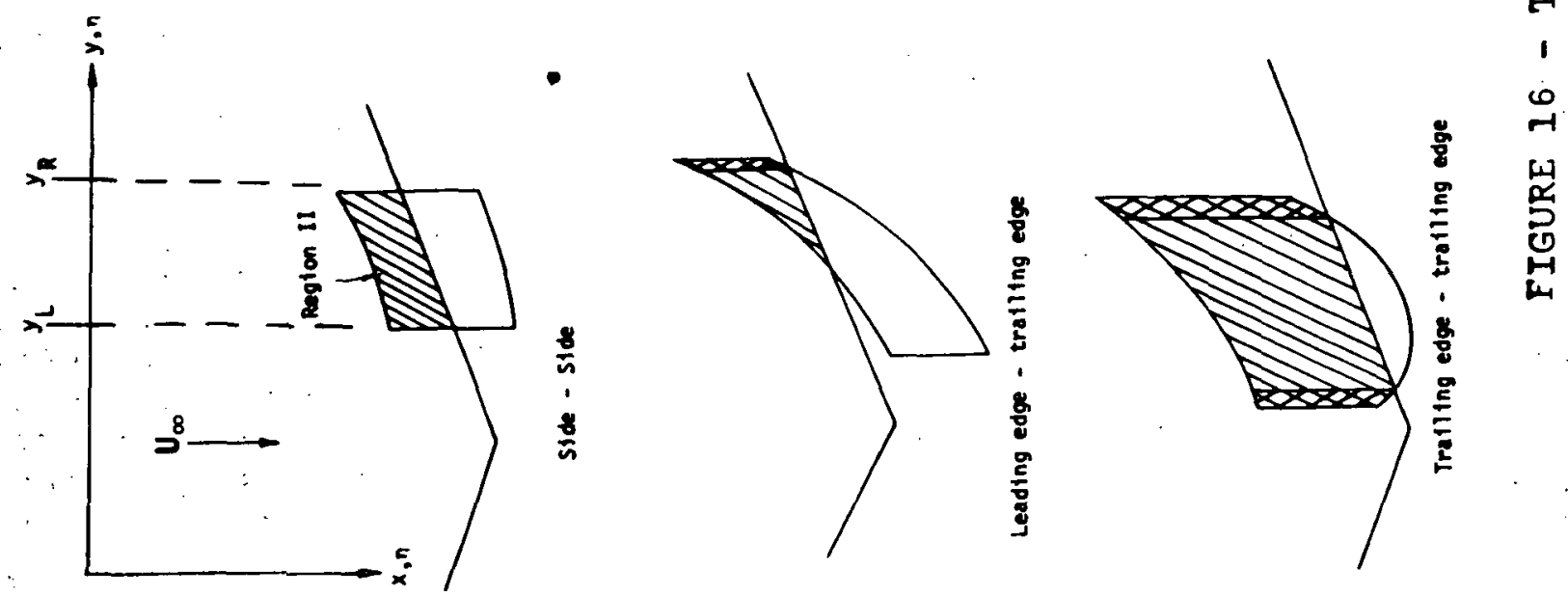
The four roots of this experession ( $t$ wo for the $+B$ and two for the $-\beta$, represent the four possible intersections of the panel leading or trailing edges and their extensions with the Mach line. A total of eight roots are obtained wen both leading and trailing edges are considered. The scheme to identify the regions and liaits on $n$ takes these eight roots and arranges the so that they are in order according to their $n$ value (fro left to right). vith each root is an identifier which states whether the root belongs to the leading or trailing edge. Roots to the left cf the left panel edge $\left(y_{L}\right)$ are excluded as well as roots to the right of the right panel edge $\left(y_{R}\right)$.

The integration limit determination procedure starts by establishing uhether the Mach line crosses the left panel edge ahead, behind, or on the panel. If ahead of the panel, the first region, bounded by $y_{\text {, }}$ and the first intersection (uhich has to be with the leading edge) will be a Region 0 (no integration - no influence). If on the panel, the first region uill be a Region II. If behind the panel, the first region will be a Region I. If there are no intersections (roots) betveen $y_{L}$ and $y_{R}$ then the entire panel is of the same region tyce. The key region is Region II. Fror this region, the next region will be either 0 cr. I depending wether the intersection is with the leading or trailing edge respectively. In going from one region tc ancther, Region II vill always be involved.

Figure 17 shows the logic block used to establish the limits. once the procedure is started, at the left panel edge, it proceeds automatically with $y_{L}$ being the first limit and $y_{R}$ being the last. As an example of how the logic block results are used, Pigure 17 also shows the regions and $n$ limits of integration for a right going Mach line whose origin is positioned behind the left panel edge ( $R_{1}$ denotes a root cf Equation (53)).

one quick test which can be made to see if a panel has any influence on a control point before using the above logic is to check the $x_{1}$ value versus the wost forward $\zeta$ value of the panel edge. If $x_{1}$ is upstream of this $\zeta$ value then the panel cannot influence the control point regardless of the supersonic Mach number. This computation can save evaluating the sorting roots in many instances.

Because the supersonic flow problen requices this added computation. which involves only the panel-control pcint relationship. and since each panel is covered by several $Y_{j}$, it is advantageous to integrate all the possible components of each spline function which share a particular panel-control point relationship. That is, the contributions to Alf from each of the tuelve components of $r_{j}$. in (Equation (25)) are not summed consecutively, but rather are added to as the program loops from 

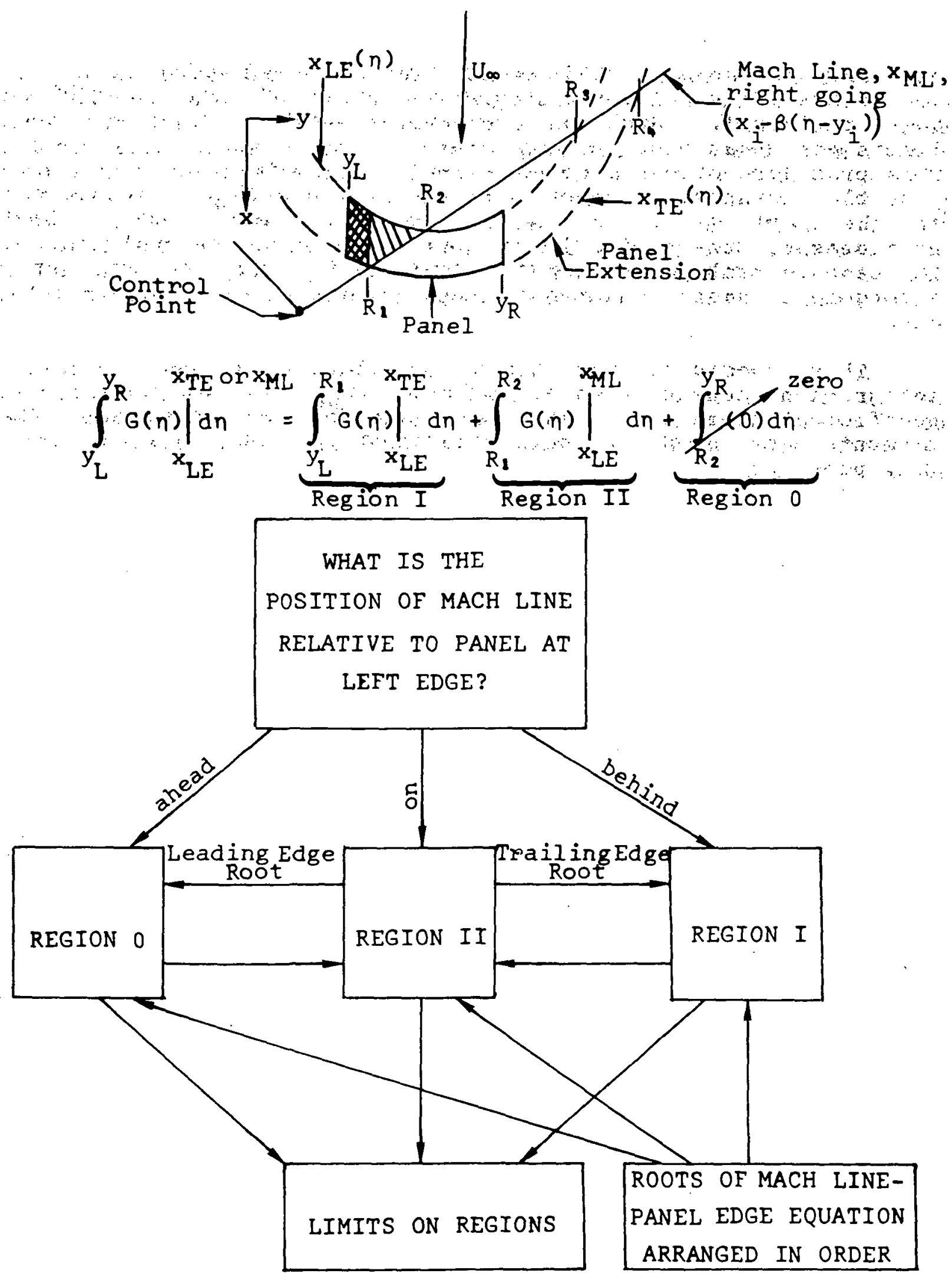

FIGURE 17 - LOGIC BLOCK TO SET LIMITS OF SUPERSONIC INTEGRALS 
one:panel to another. This weans that the total value of $A_{1 j}$ may not be available untilall integrationsover all the panels have been performed. Such an arrangement avoids setting the region limits more than once for any control point-panel relationship. This procedure offers a computational speed advantage over ancther possible arrangement wich forms one complete $A_{1} s$ before going to the next (i.e.. a complete $Y_{j}$ ). With this latter arrangewent, the region liaits must be cosputed several times for the same control-point panel relationship. due to the spline arrangement where functions occupy common panels (Figures 10 and 12).

This concludes the basic formulation of the numerical integration scheme used to evaluate $A_{11}$ (termed the influence coefficient elements). The next section describes how these elements are used to match the boundary conditions for various flow problens. 
5.4 Satisfaction of Boundary Conditions on Singularity Surfaces

The preceeding sections showed the construction of the basic building block of the numerical scheme - the vortex spline: This section shows how the strengths, Sf (multiplicative constants of each $\left.r_{f}\right)$, are determined by apflication of the boundary conditions on the singularity surface (region "s" of tquation (11))." Each elenent naturally satisfies the governing differential equation, since they were developed from the horseshoe vortex, and each element also satisfies all the continuity of vorticity required of the final solution (the purpose of the spline), therefore the strengths of these elements are all indefendent. This independency weans that every element can be separately adjusted such that it best satisfies the imposed boundary conditions. Since the splines have a limited number of degrees of freedom, they, in general, cannot exactly satisfy these boundary conditions. A compromise match must te accepted. The comfrcmise used for the spline developrent was a least square error match. A solution based on this technique will yield the best overall fit to the boundary conditions.

The square error involved in the approximation to the boundary conditions can be mathematically expressed as:

$$
E^{2}=\iint_{s}\left[w_{B C}(5, n)-w_{S}(5, n)\right]^{2} d 5 d n
$$

where $E^{2}$ is the square error, $W_{B C}(\zeta, n)$ is the applied downash boundary condition and $W_{S}(5, n)$ is the downwash from the splines. since it is not possible to perform the indicated surface integration for general cases, an approximation has to be wade. This is done by assuning the integral can be expressed as a weighted sum:

$$
\left.E^{2} \cong \sum_{l=1}^{M}\left[w_{B C}\left(\zeta_{1}, n_{l}\right)-w_{B} \zeta_{l}, n_{l}\right)\right]^{2} a_{l}
$$

Here $\left(5_{1}, n_{1}\right)$ is one of $M$ points on the surface (termed the control pointst" and a is an area associated with that pcint. (The choice of control point locations and their associated areas is discussed later.) Equation (16) can be used to express $W_{S}\left(s_{1}, n_{1}\right)$ in Equation (55) giving:

$$
E^{2} \equiv \sum_{l=1}^{M}\left[w_{B C_{1}}-\sum_{j=1}^{N} A_{l j} s_{j}\right]_{a_{l}}^{2}
$$


The notation has been abreviated such that the subscript i denotes the value at $\left(r_{1}, n_{1}\right)$.

The square error in Equaticn (56) can be minimized with respect to each strength, $s_{j}$, since these strengths are independent. This minimization is accomplished by differentiating Equation (56) with respect to $s_{k}$ and setting the resulting expression to zero:

$$
\frac{\partial E^{2}}{\partial S_{k}}=2 \sum_{l=l}^{M}\left[w_{B C_{l}}-\sum_{j=l}^{N} A_{l j} S_{j}\right] a_{l} A_{l k}=0
$$

The variable, $k$, ranges from 1 tc $N$, therefore Equation (57) represents $N$ algebraic equations which can be solved for the $N$ strengths. A more useable form for Equation (57) is:

$$
\sum_{l=1}^{M} \sum_{j=1}^{N} A_{l k} a_{l} A_{l j} s_{j}=\sum_{l=1}^{M} A_{l k} a_{l} w_{B C_{l}}
$$

The entire set of Equations (58) can be written in matrix notation as:

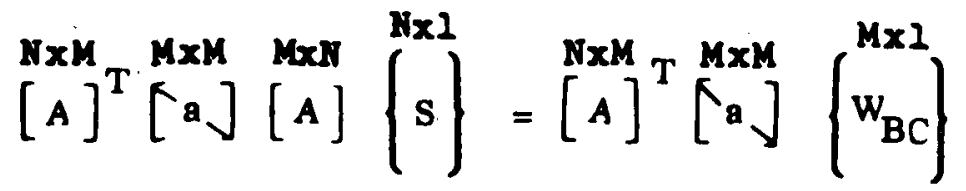

Here $[A]^{\mathbf{T}}$ is the transpose of matrix $[A]$ with elements A 1 and $[a]$ is a diagonal matrix with $\in$ lements a, The \{S\}, and the are vectors refresenting the unknownstrengths, (59) for (\$) gives:

$$
\{s\}=\left[[A]^{T}[a][A]\right]^{-1}[A]^{T}[a]\left(w_{B C}\right)
$$

Fquation (60a) is valid wenever the number of control points is grcater than or equal to the number of unknowns ( $M \geq N)$. If the number of control points is equal to the number of unknouns $(M=N)$ then [A] is a square matrix and Equation (60a) reduces to the determinate form of:

$$
\{8\}=[A]^{-1}\left(W_{B C}\right\}
$$

An abreviatea notation is introduced here: 


$$
\begin{aligned}
\tilde{A}^{\mathrm{T}} & \equiv[A]^{\mathrm{T}}[\mathrm{a}] \\
A & =[A] \\
\dot{s} & =\{s \\
w_{B C} & =\left(w_{B C}\right)
\end{aligned}
$$

then

$$
S=\left(A^{T} A\right)^{-1} A^{T} \quad W_{B C}
$$

The symbol $\tilde{\mathbf{A}}^{\mathrm{T}}$ is the transpose of A veighted by [a].

When the special Mach line paneling is employed, the hyperbolic nature of the flow can be used to advantage by treating each region as a separate wing and solving the problem in steps. Fiqure 18 shows the arrangement of the pasels with the associated [A] matrix partitioded into elements which represent the influence of subdivisions on themselves, $A$ and elements which represent the influence of subdivisions cn other subdivisions. B. It is obvious from this figure that it is very inefficient to solve this problem with this one large matrix. Instead, the problem can be divided into three separate, smaller froblems. corresponding to the three subdivisions (I, II, III). If this is done, the three equations to be solved in a manner analogous to Equation (59) are:

$$
A_{I} S_{I}=w_{I}
$$




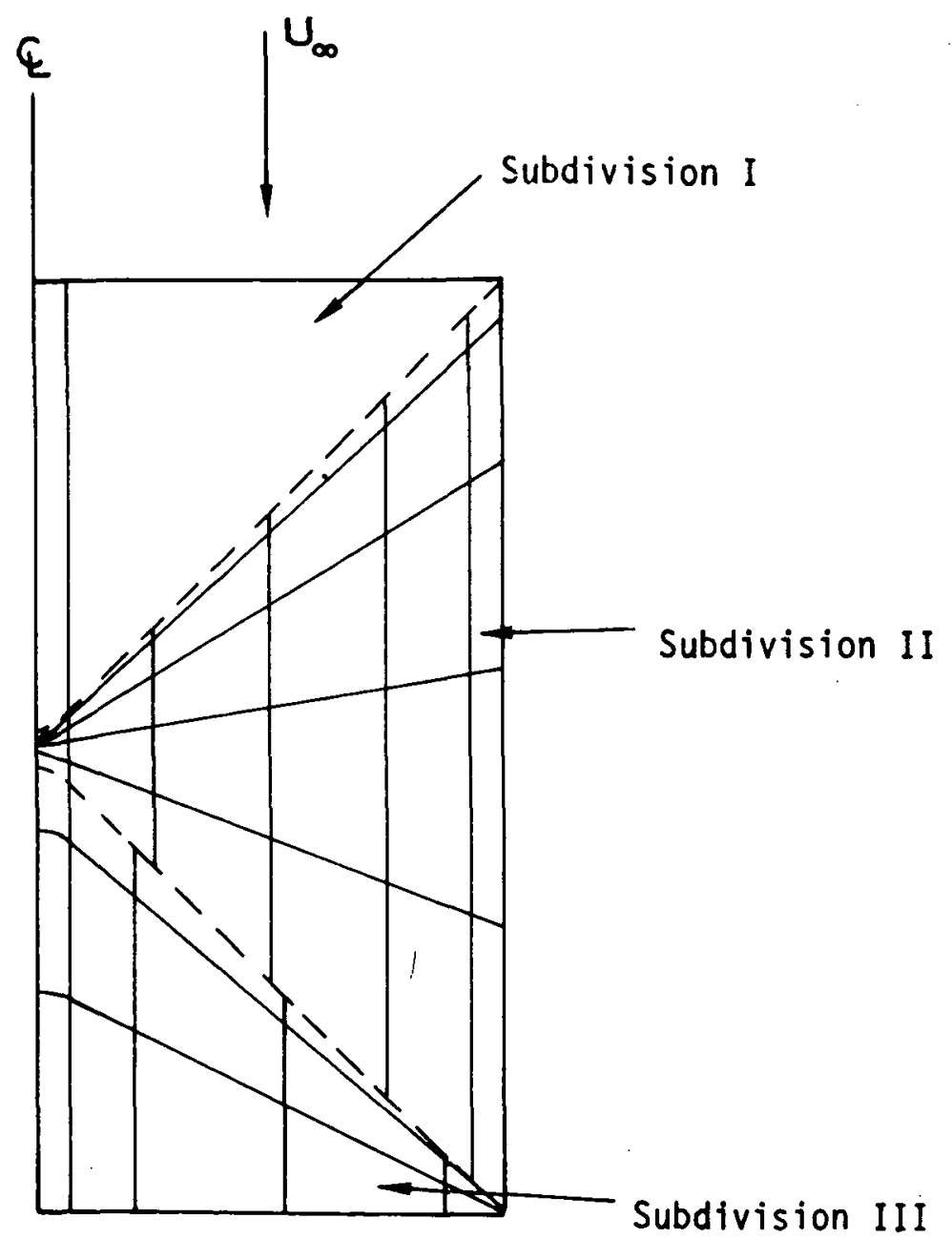

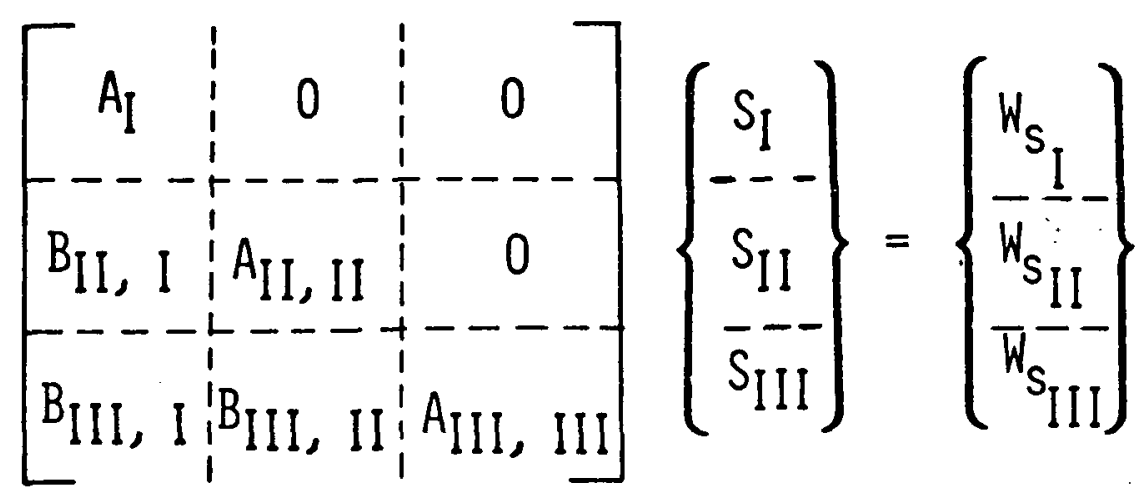

FIGURE 18 - PARTITIONING OF AIC MATRIX FOR MACH LINE PANELING 


$$
\begin{gathered}
A_{\mathrm{II}} s_{\mathrm{II}}=w_{\mathrm{II}}-w_{\mathrm{II}}^{\prime} \\
{ }_{\text {III }} s_{\text {III }}=w_{\text {III }}-w_{\text {III }}^{\prime}
\end{gathered}
$$

The subscripts denote the subdipisions and the quantities " $\nabla_{I I}$, and "m are the known downwashes in each subdivision. The right hand sides of Equation (63b) and (63c) can be thought of as "effective" downwashes for the independently treated dowstream subdivisions.

Another computational consideration is the cost of performing the operations indicated in Equation (60). A more. direct method can be employed if Equation (56) is rewritten as:

$$
E^{2}=\sum_{l=1}^{M}\left[w_{B C_{l}} \sqrt{a_{l}}-\sum_{j=1}^{N} \sqrt{a_{l}} A_{1 j} s_{j}\right]^{2}
$$

The above expression can be minimized directly using Householder's technique (Ref. 9). This procedure provides a great savings in computation time over the method represented by Equation $(60)$.

The key feature of this least square error approach is to select the poiuts $\left(\zeta_{1}, n_{1}\right)$ and the areas, $a_{1}$. such that Equation (55) does approximate Equation (54). With this goal, a study of various arrangements was undertaken. Fundamentally the study entailed subdividing the panels, which were used to form the splines. These subdivisions were formed by lines of constant percentage span and chord. Contrcl pcints were located on these subdivisions using the area of the subdivision as the weighting factor, a . in Equation (55). These points were located at the spanuise centroids of the subdivisions, widway between the leading and trailing edges. In the study the number of spanyise sections could be specified independently from the chordwise sections. 
Figure 19 shows the minimum arrangements resulting from the study for supersonic and subsonic flom. By minimum, it is meant that the arrangement's approximation to the integral (Equation (54)) was adequate over a wide variation of paneling. (Results presented later will show the accuracy of the arrangements for cases with as few as twelve panels and twenty-four control points.) The supersonic case required more control points because of the linited extent of influence in sufersonic flow; consequently wore points are needed to "sense" this influence.

The study also revealed that difficulties are encountered in the vicinity of subsonic leading edges and at all tips due to the singular nature of the vorticity distribution in these regions. Since the splines do not have the proper asymptotic form, the downwash has an inproper singular behavior. This downash departure tends to have a strong influence on the error inimization procedure if control foints come too close to the edges involved. So, another study was ade tr determine suitable locations for control points in these regions. A review of other iifting surface schenes shored that their respective trodimensional formulations could give the froper lift on a flat plate at angle of attack in subsonic flow using only one element (e.g., $N=1$ in Equation (16)). In fact, this is a criterion that can be used to establish the proper control point location for a determinate numerical formulation (where the number of unknown strengths. is equal to the number of control points). This two dimensional criterion can be shown to be valid for the vortex lattice. (Ref. 10 ) and the constant pressure panel schemes. Using this criterion, the proper control point location for the spline was computed. The one element spline representation for the lift on a flat plate is shown in Figure 20. The integrated load for the spline vas set equal to the linear theory integrated load. The Iocation was then found where the downash from the spline is equal to the downash on the flat plate. This location is at the center of the panel. (This result can be confirmed using Equation (18) with the distribution $r(\zeta)=\left(x_{2}-\zeta\right) /\left(x_{2}-x_{1}\right)$ ). starting uith this result, it was discovered, by numerical investigation, that points on or downstream of the center chord line of the leading edge panel consistently produced accurate results. Since the components of the spanwise functions begin and end at the mid panel span. (Fiqure 10), the criterion in this direction is that the control points near an eage be on or inboard of the three quarter panel span (corresponding to the center of the half panel).

Using the above criteria, the general paneling arrangements shown in Figure 8 could be applied directly without any special considerations for subsonic flow, since the control foint configuration satisfies the conditions outlined. For supersonic flow, there is no problem spanuise; chordvise, with a supersonic 


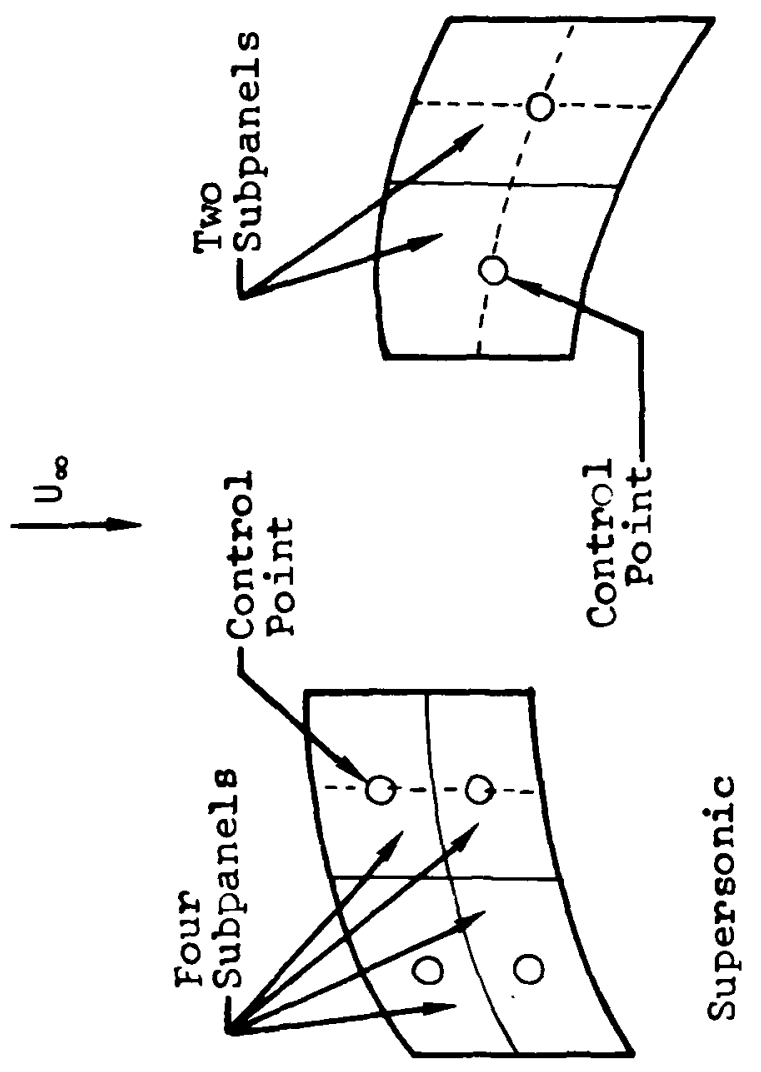

㟧

톰

1

क

宸 

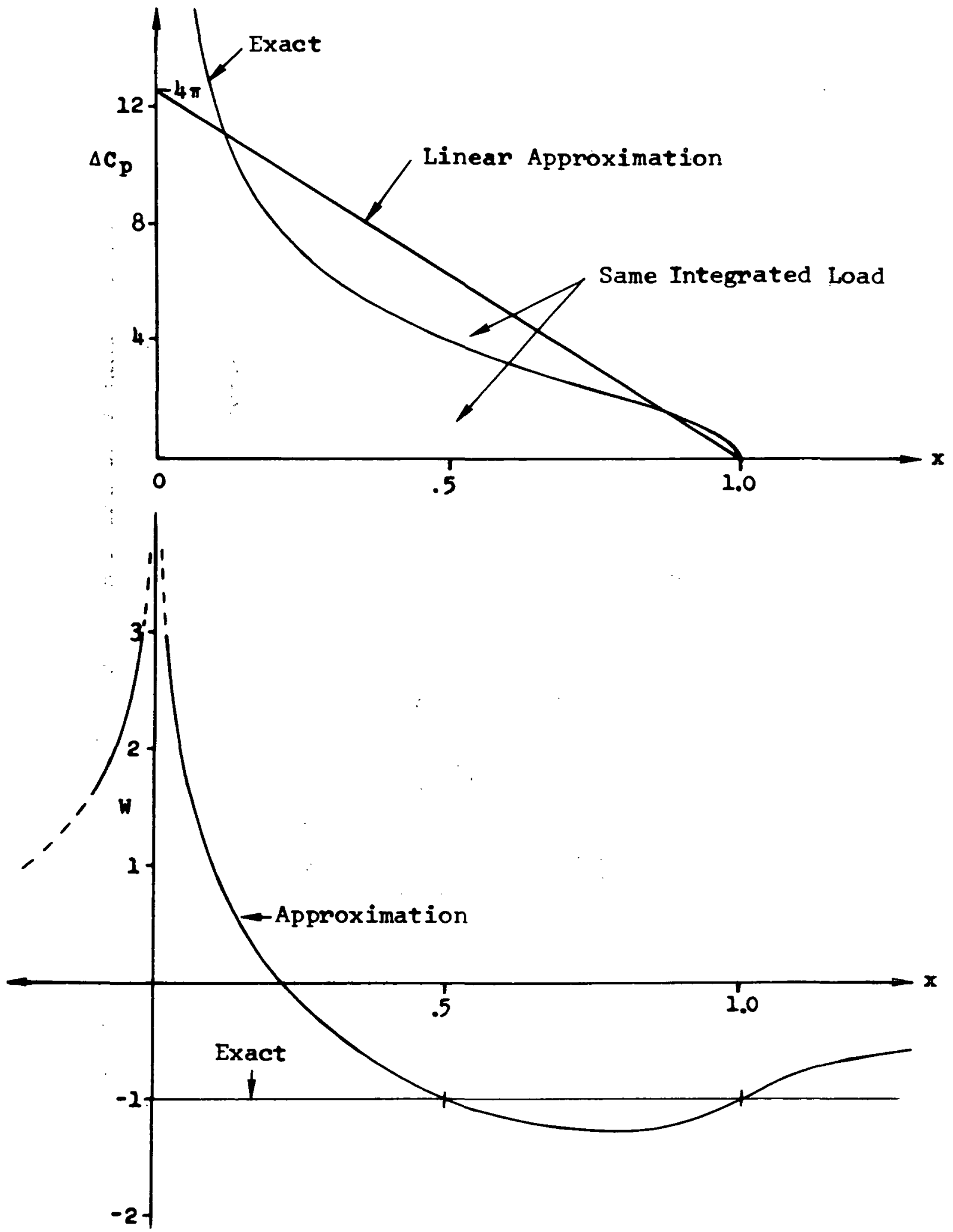

FIGURE 20 - TRIANGULAR PRESSURE DISTRIBUTION MODEL OF A FLAT PLATE AT ANGLE OF ATTACK 
leading edge, there is also no problea. But, if the leading edge is subsonic, the tro forward control points must be excluded frow the minization process. With this simple exclusicn rule. consistently accurate results were cbtained over a wide range of test cases.

The benefit of this least square error scheme can be more fully realized for cambered surfaces where the numer of functions needed to model a problem can be saall compared to other schemes. This is because relatively fev functions can model rather rapid camber variations and the number of functions is not tied one to one to the numer of control points. With the least square error formulation, any number of control points per panel can be introduced to accurately incorforate the camber variation into the problem and at the same time, the number of functions can be held to a ninimu. As pointed out earlier, the configurations shoun in Figure 19 represented the minima arrangenent for consistent accuracy. any larger number of control points could also be used to obtain better camber definition, (subject to the edge constraints outlined abovel. 


\subsection{Computational procedure}

Pigure 21 shows a flow diagram of a method to compute results using the vortex spline scheme. Once the strengths, $S$, have been computed, the remaining parameters can be determined le.g.. lift, monent, pressure distribution, etc.). Since the splines provide a continuous distribution over the surface, the fressure and bound vorticity can. be evaluated directly at any point by deternining the aggregate influence of the contributing functions. This continuity also makes it possible to conpute the shed vorticity, $\sigma$, by integrating and differentiating the basic distribution for $r$ as indicated by Equation (23).

The lift and load distribution can be computed either by actual1y integrating the basic functions or by pertorming a sum on the pressures. similar to wat was done for the least square downush error integral formulation using the same control pcints and areas. The moment can likewise be computed by integrating or suming; hovever, the accuracy of this calculation using the suning technique is less than that for the lift calculation. Nevertheless, the suming technique is sufficiently accurate when several panels are enployed and was the method used to compute both the lifts and moments presented in the next section.

In addition to the standard aerodynamic paraneters, the mean square error can also be computed. This mean is the paraneter. E? in Equation (55) divided by the total area (sum of the a). The mean square error (with appropriate control point exclusion) is an excellent indicator of the overall accuracy of the solution. The square root of this value, the rcot mean square downwash error. was found to be the same order of magnitude as the root mean square pressure error for the cases exanined. This RMs error parameter supplies the user with an a posterior judgment of the validity of a flow nodel without the need to consult other data. It also opens the possibility of formulating a scheme which vould autonatically adjust the paneling and rerun a case if sufficient accuracy were not obtained fron the initial run.

The next section presents the test cases that vere used to evaluate the schene. 


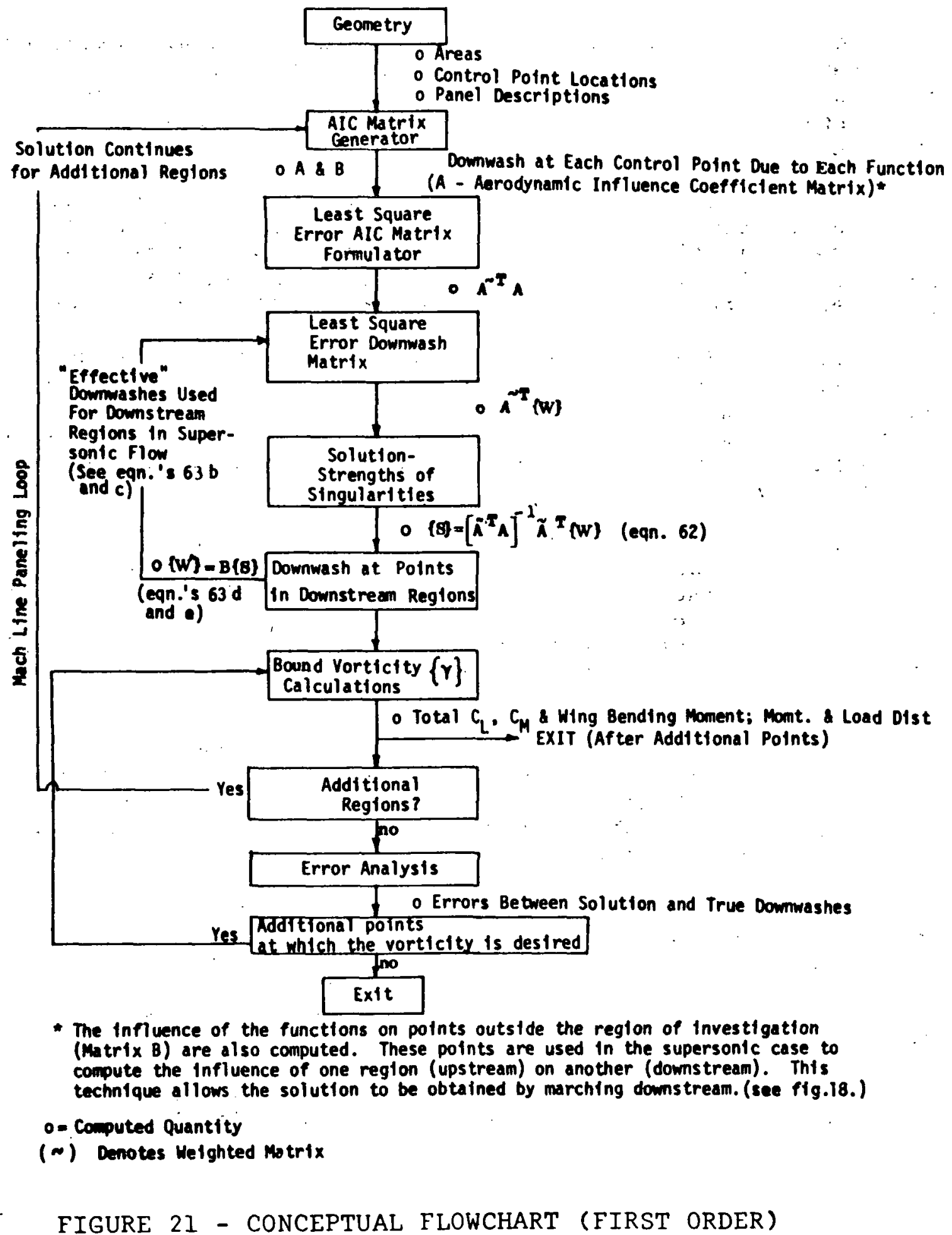




\subsection{Vumerical Besults}

Various configurations were analyzed to determine paneling requirements and convergence to a known answer. The spline function results were compared with other lifting surface schemes and exact, linearized flow solutions. Figures 22 through 28 show the results.

Figure $22^{*}$ is a comparison of the spline schene with another lifting surface solution (Ref. 10) for a circular wing in incompressible flow. The comparison shows good agreement not only for the coefficients but also for the detailed loading. Note how well the quadratic edged panels can watch the exact planform.

Figures 23 and 24 show comparisons of the spline scheme with a pressure mode scheme (Ref. 2) for compressible, subsonic flow. Again agreement is very good. Note the rounding of the planform representation at the root for both cases. Thiseliminated downwash singularities vithout introducing any special singular forms for $r$.

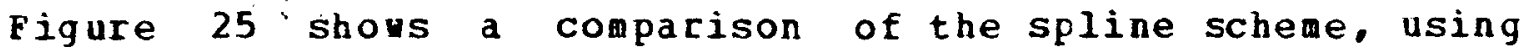
special Mach line paneling. with the exact, conical flow result. (Bef. 11). The plantor paneling shows a rounding at the root. This rounding produced a slight departure of the pressure from the axact result in the vicinity of the rounding (not noticeable in the chordwise plot of the pressurel.

Pigure 26" 'shows two comparisons of the spline scheme using geometric paneling for supersonic flow: one with the exact solution and one with a constant pressure panel scheme using the same paneling. Even for this faneling, which ignores the special Mach lines, the spline scheme gives accurate coefficient results with a reasonable comparison tc the fressure.

Figure 27 shous another comparison of the spline scheme with exact conical flow results (Ref. 11). This planform has a subsonic leading edge. Again note the rounding of the planform. This rounding causes a local departure of the pressure from the exact result as shown. The overall agreement is good, even for the relatively fey panels used.

Figure 28 shows the numerical stability of the formulation for various paneling schemes. In all cases the pressure distributions appear to be as accurate as could be expected for a 

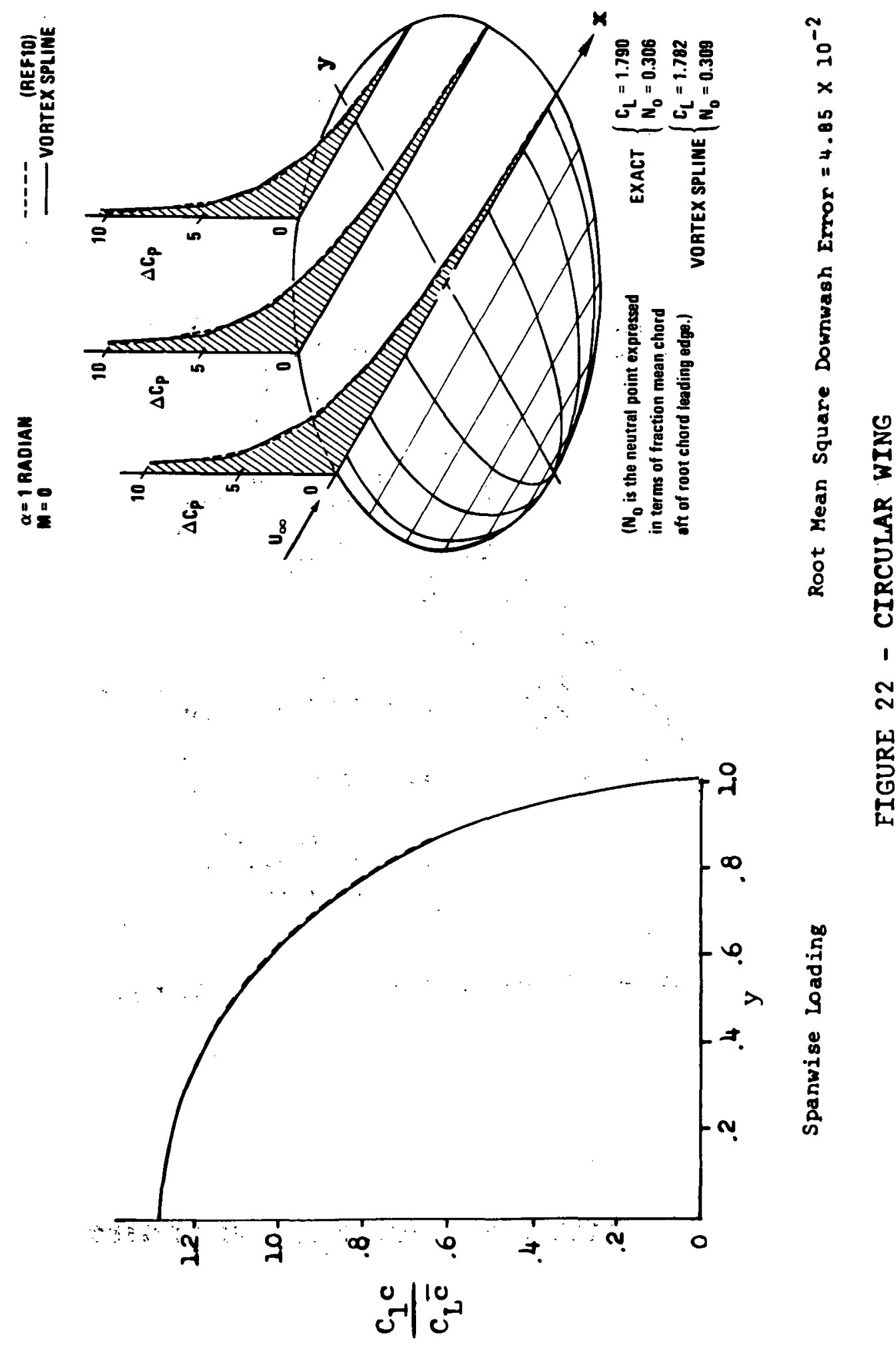

崩 
$\because$ VORTEX SPLINE -

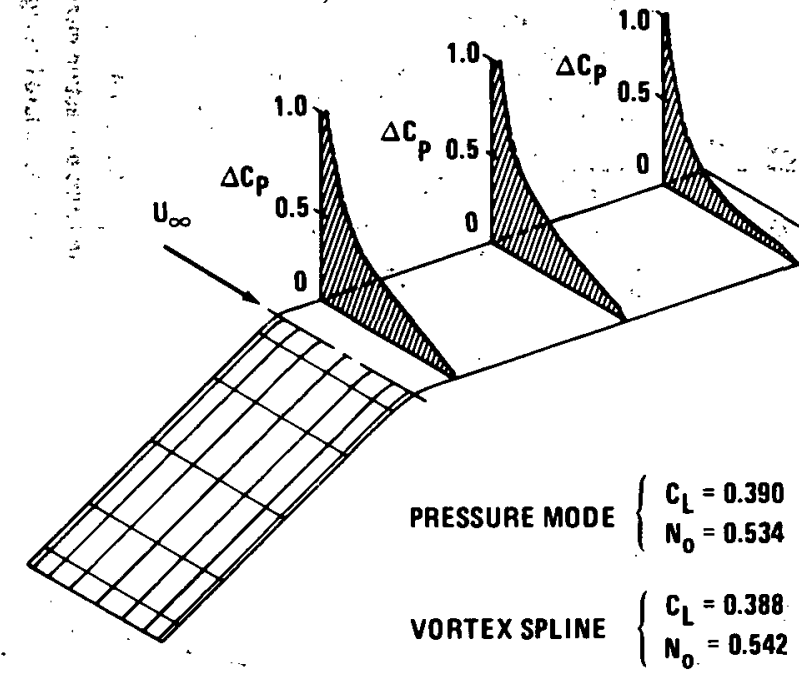

( $N_{0}$ is the neutral point expressed in terms of fraction chord aft of root chord leading edge.)

Root Mean Square Downwash Error $=2.15 \times 10^{-2}$

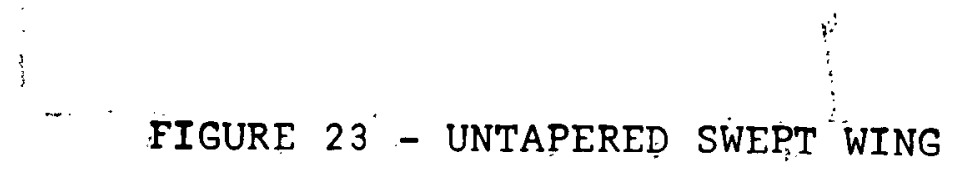




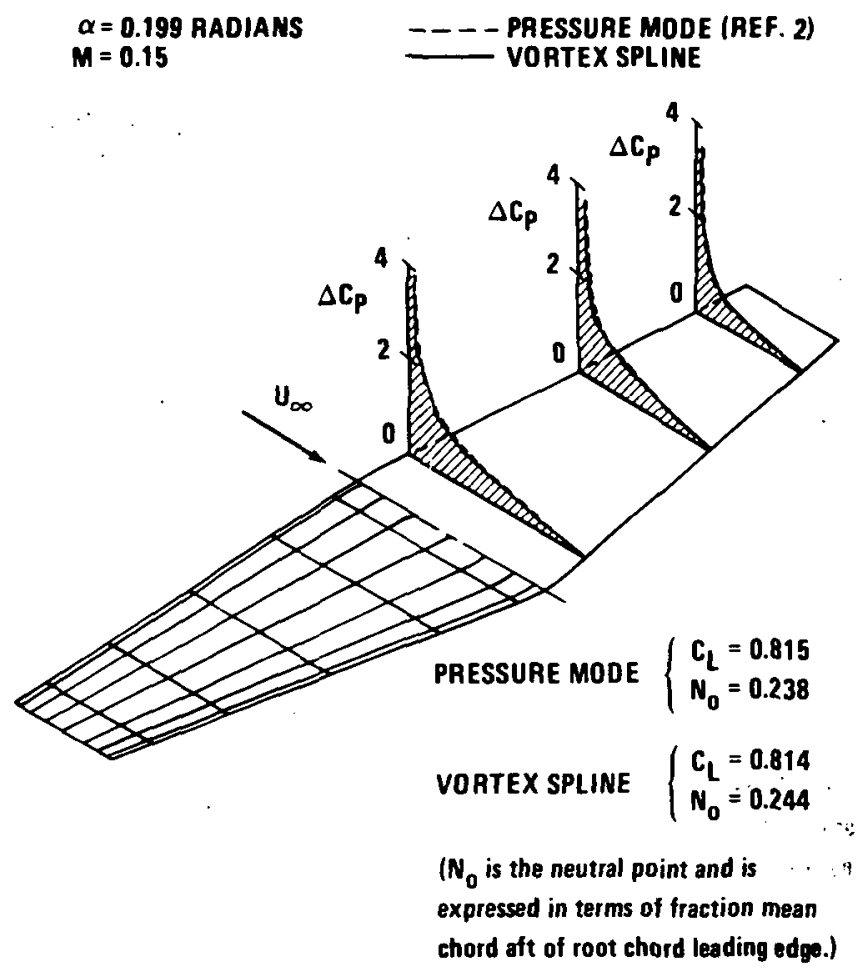

Root Mean Square Downwash Error $=1.81 \times 10^{-2}$

FIGURE 24 - TAPERED WING WITH ZERO SWEEP OF QUARTER CHORD 


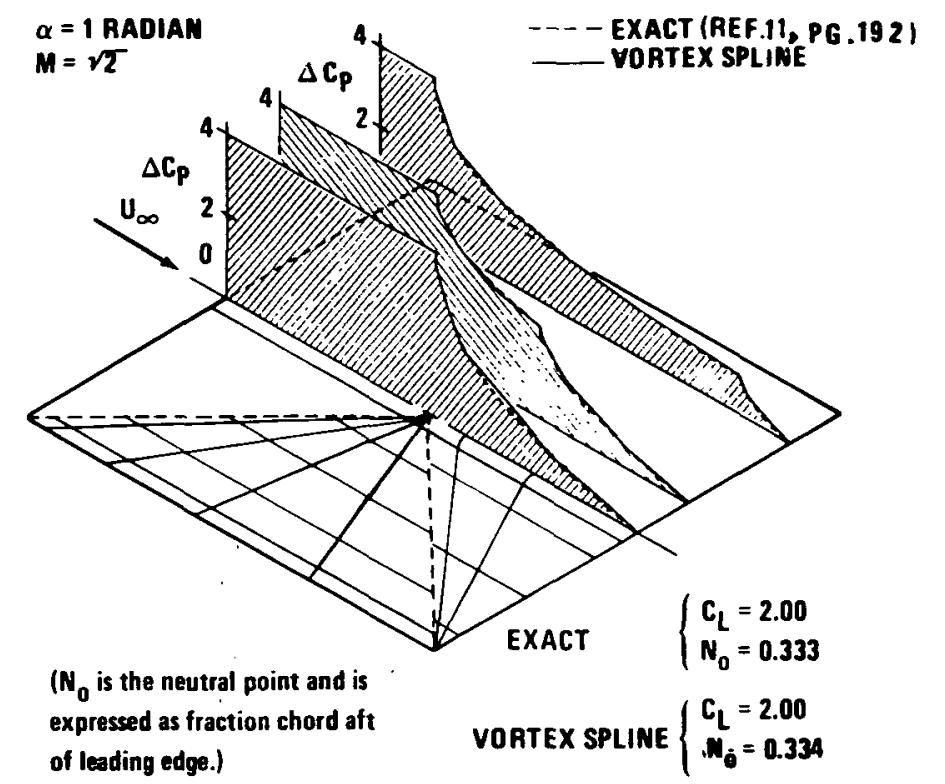

Root Mean Square Downwash Error $=4.63 \times 10^{-3}$

FIGURE 25 - SQUARE WING (SPECIAL MACH LINE PANELING) 

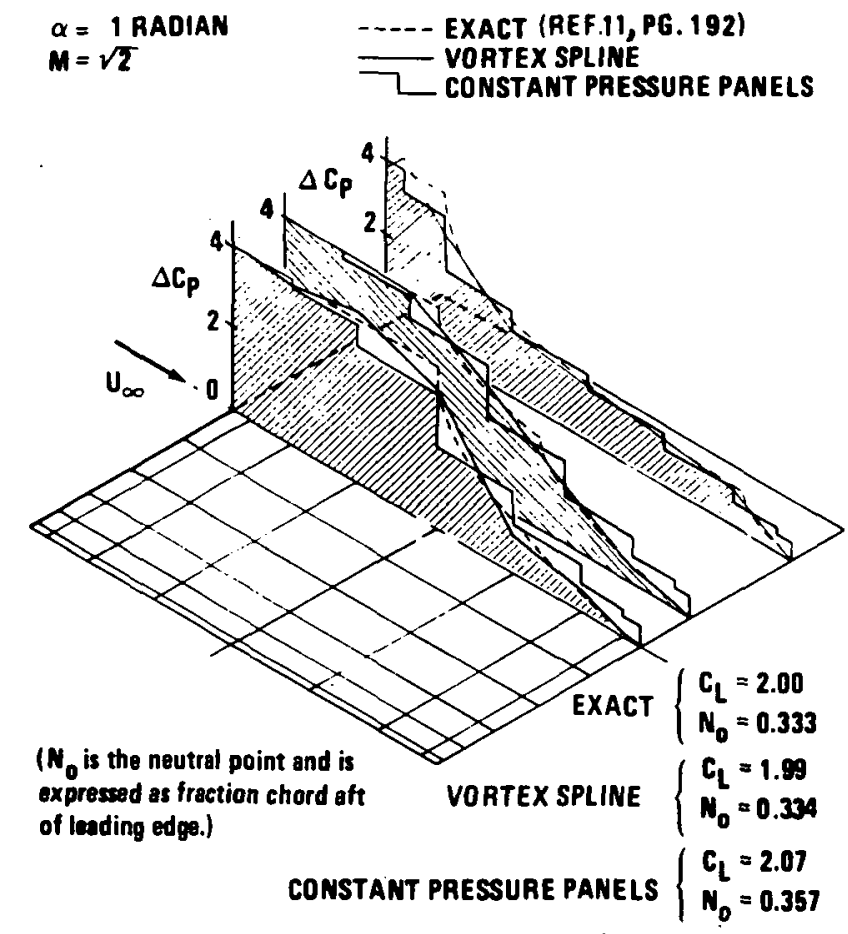

Root Mean Square Downwash Error $=1.79 \times 10^{-2}$

FIGURE 26 - SQUARE WING (GEOMETRIC PANELING) 

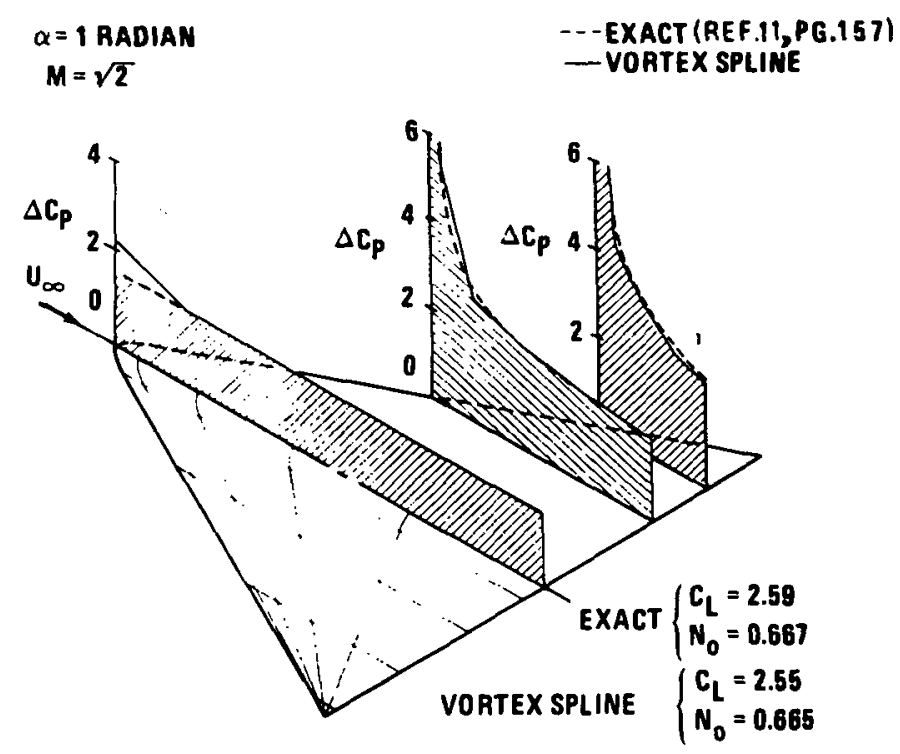

( $W_{0}$ is the neutral point and is expressed as fraction of man chord aft of apex.)

Root Mean Square Downwash Error $=5.00 \times 10^{-.2}$ 

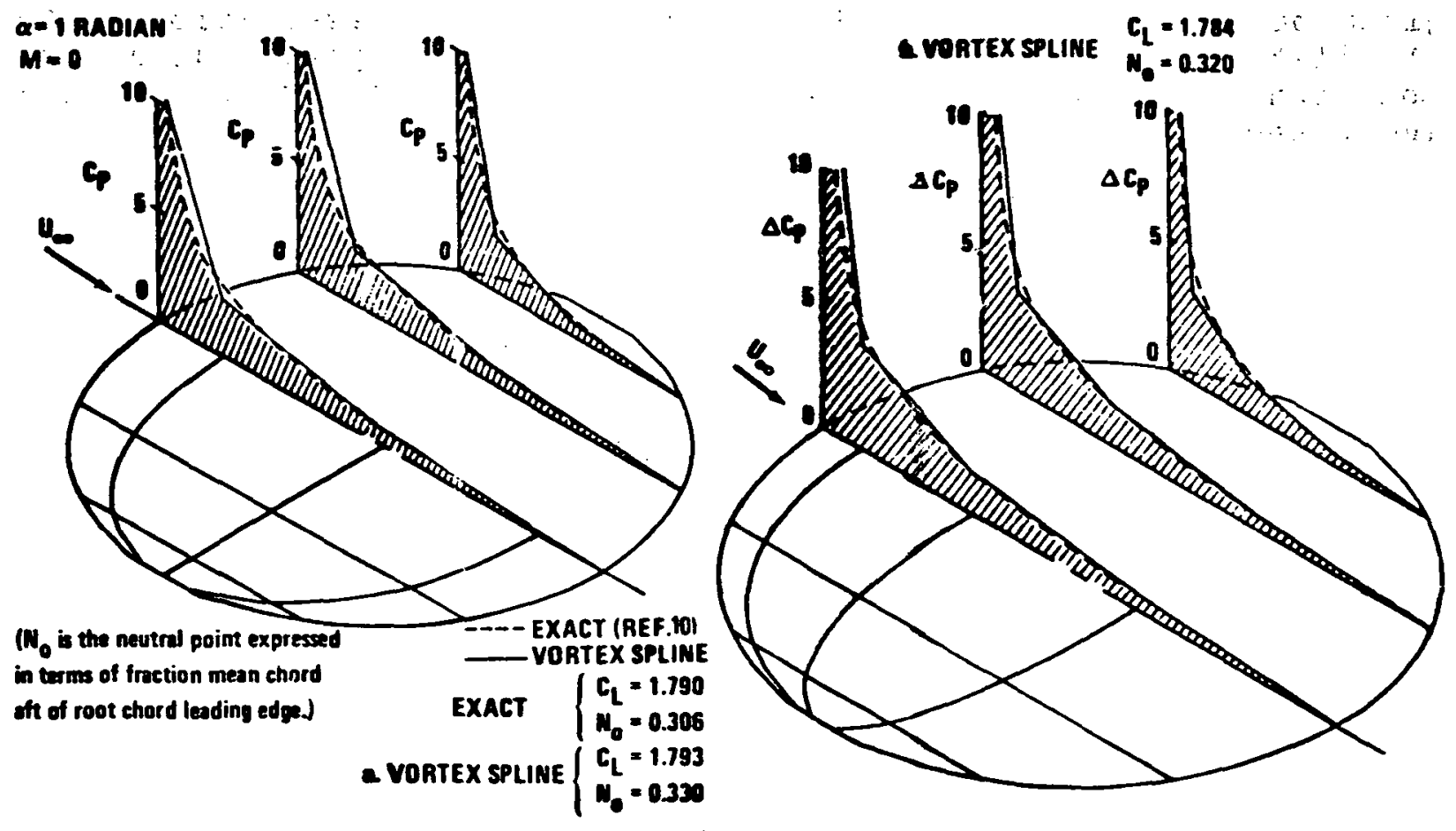

Root Mean Square Downwash Error $=9.65 \times 10^{-2}$

Root Mean Square Downwash Error $=1.02 \times 10^{-1}$

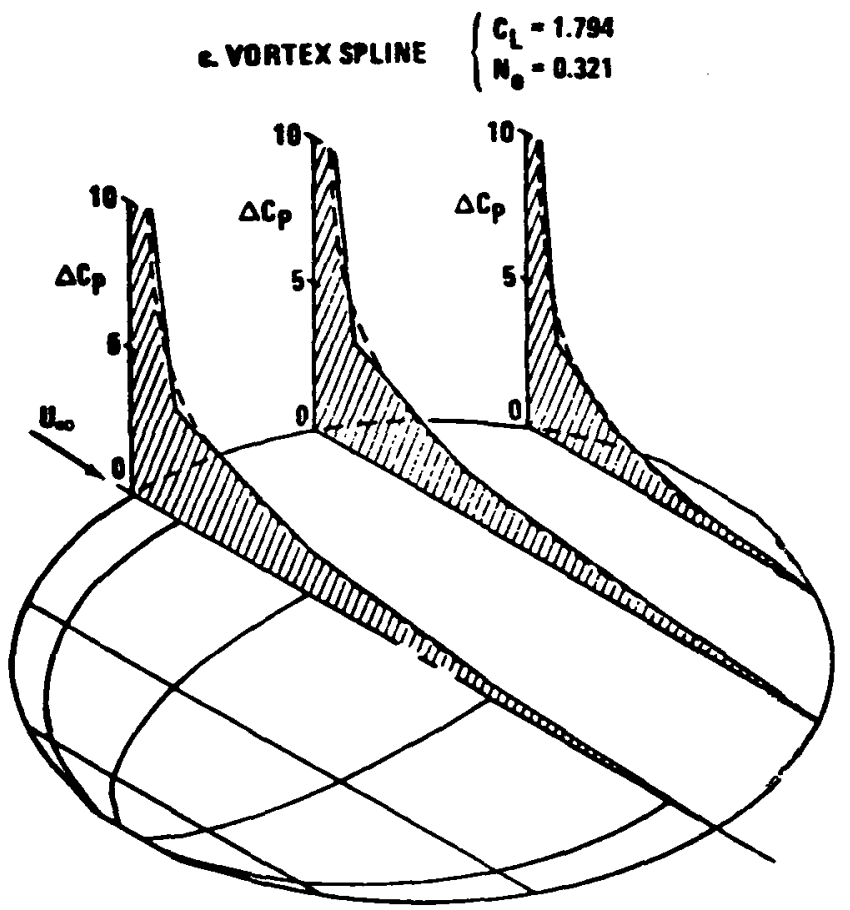

Root Mean Square Downwash Error $=1.02 \times 10^{-1}$ 
given paneling using a linear chordwise representation. The stability of the solution does not depend on paneling, and the soluticn converges to the exact results as the number of panels is increased. 


\subsection{SHELL REPRESENTATION USING, THE SPLINE, SCHEME}

\subsection{General Apfroach}

This section presents a scheme which represents the interference effects on ving-body combinations. The basis for this representation is presented in Reference 1 where it is referred to as an interference shell. The shell amounts to a cylindrical surface with singularities distributed over it, much the same as for the wing. The iaterference shell, however, is not a impermeable surface with flow tangency boundary conditions. The purpose of the shell is to account for the wutual interaction between the body and the wing. The shell boundary conditicn is that the shell singularities induce a velocity which cancels the velocities on the shell that are induced by the wing. The body contained inside the she11. will however. induce a flow through the shell which is not canceled. The enrlosed body then appears to be in an undisturbed free strean flow. therefore its analysis can be treated separately using some appropriate technique (e.g... Ref. 121.

The aim of this shell study was to provide an improvement over the constant pressure panel shell (Ref. 1). In so much as the vortex spline arrangement provided much improvement over the constant pressure panel scheme for the wing, it was felt a similar development for the interference shell would be appropriate. There was, however, an unresolved question concerning the interference shell - namely whether the elementary horseshce vortex was the appropriate singularity needed to model the bodywing interaction effect. The quostion arose because bodies in potential flow do not have a wake (i.e.. shed vorticity) and because the elementary horseshoe vortex does have a wake. This difference between the flow $c n$ a wing and the flow cn a body can be attributed to the roundness of the aft end of a body as compared to the sharpness of the trailing edge of a wing (suggesting no kutta condition on a body). If the Kutta condition is not used, a vortex solution is not unique (Ref. 5, pg. 57). This means that a shell representation using the vortex spline which needs the kutta condition might not properly model the flow on a hody. Because of this pcssibility, a study was initiated whereby several elementary sinqularities were develcped which could be utilized in a spline technique. These singularities were the elementary norseshoe vortex. the source, and the dourlet. Unfortunately, none of the analyses was developed to the point of yielding numerical results. The next section shows the analysis which was performed for the various singularities as well as a detailed description of a scheme using the elementary horseshoe 
vortex in an influence coetficient approach. The scheot described is compatible vith the scheme already fresented for wings. 


\subsection{Interference shell singularities}

Figure 29 shows the cylindrical coordinate system used to analyze the interference shell. Since the doublet and elementary horseshoe vortex car be derived frow the source (Ref. 5, pp. 28 and 87), the analysis vill begin with the source singularity for subsonic flow.

The elementary source potential. $\phi_{8}$. for subsonic tlow is given as (kef. 5, pq. 87):

$$
\Phi_{8}(x, r, \theta)=\frac{-1}{4 \pi} \frac{1}{\sqrt{(x)^{2}+\beta^{2} r^{2}}}
$$

where $B^{2}=1-M^{2}$. In this expression, the source has been placed at the origin of the coordinate systen. If hovever, the source is located at some other point $\left(x_{0}, r_{0}, \theta_{0}\right)$, then the potential becomes $\theta$ dependent:

$$
\Phi_{\mathrm{g}}\left(\mathrm{x}, \mathrm{r}, \theta, \mathrm{x}_{0}, \mathrm{r}_{0}, \theta_{0}\right)=-\frac{1}{4 \pi \sqrt{\left(x-x_{0}\right)^{2}+\beta^{2}\left(\mathrm{r}^{2}+\mathrm{r}_{0}^{2}-2 \mathrm{r} \mathrm{r}_{0} \cos \left(\theta-\theta_{0}\right)\right)}}
$$

The above result can be better understocd from figure 30.

The kernel function corresponding to that used for the planar vortex panels can be derived by differentiating (66) with respect to the local surface normal coordinate (i.e... $\partial \phi_{8} / \partial \mathbf{r} \mid \mathbf{r}=\mathbf{r}_{0}$ is the downwash on a cylinder of revolution). In order to ottain the influence coefficient. Al tbis velocity kernel must be multiplied by the proper strength variaticn chordwise and circumferentially (the latter variation corresponding to the spanuise variation for planar panels). After multiplication, the function must be integrated over the domain of the singularity.

The interference shell can be panfled in a way entirely analogous to the geometric paneling scheme used for the wing (Figure 31). First the cylinder can be divided streamwise by parallel rays on the surface. Then the cylinder can be further divided by parallel planes normal to the axis of the cylinder. These cuts define a group of panels which are rectangular in the $x-\theta$ coordinates. Spline functions for the various singularities can be defined using these panels.

Since the different singularitzes require different degrees of continuity. the anaiysis fron this point or will te linited tc the elementary horseshoe vortex using the continuity conditions 


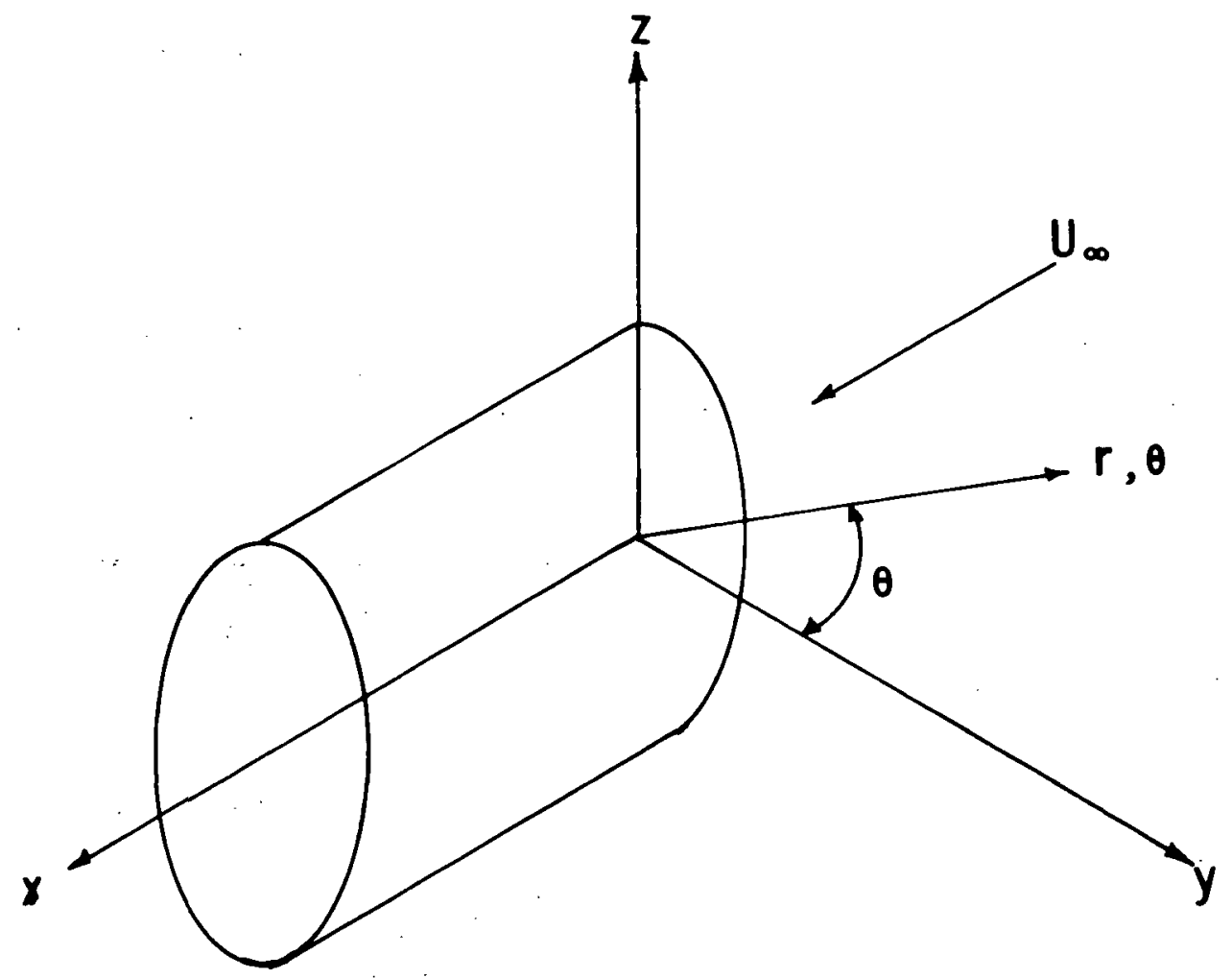

FIGURE 29 - GEOMETRY OF INTERFERENCE SHELL 


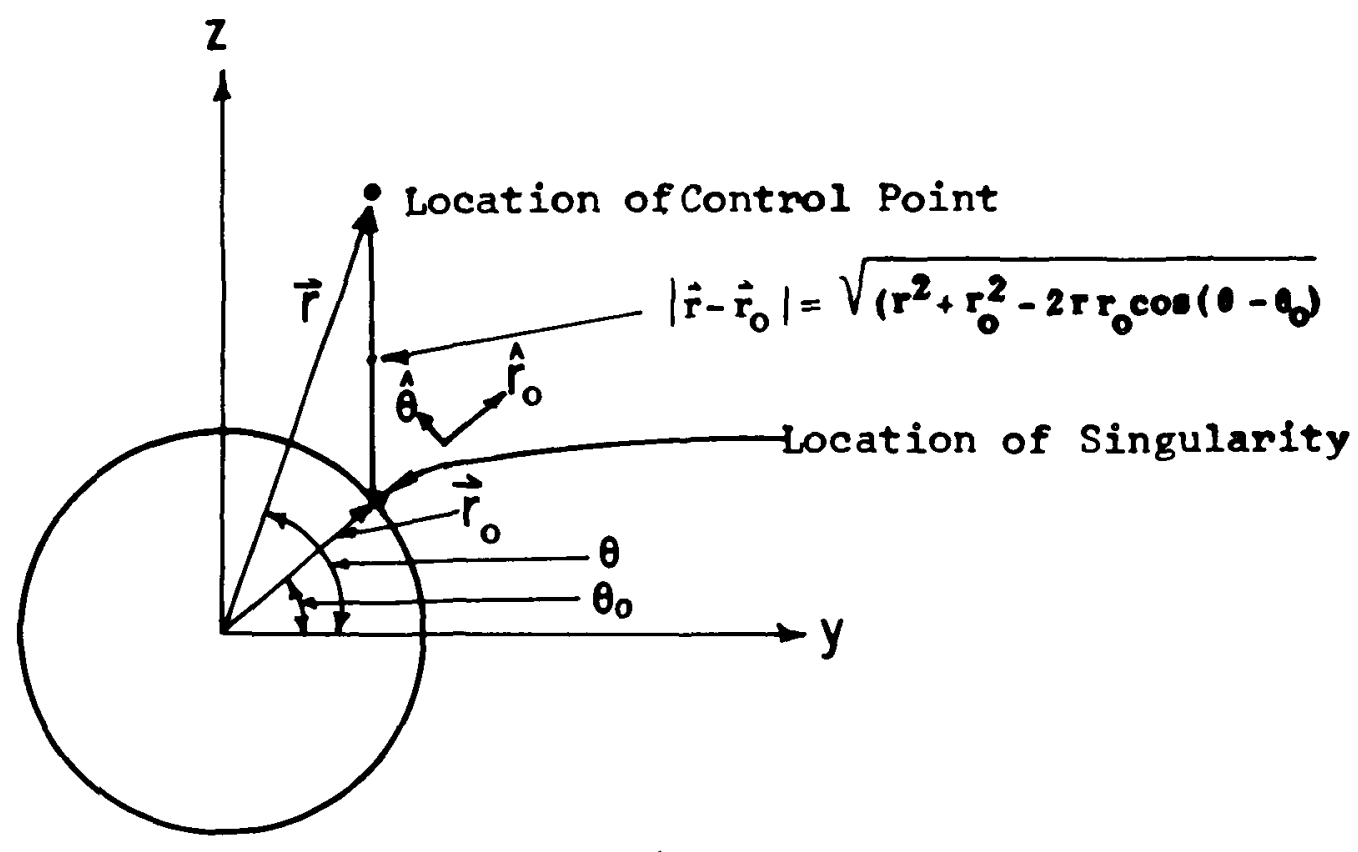

FIGURE 30 - AXIS SYSTEM AND DEFINITION OF VARIABLES 


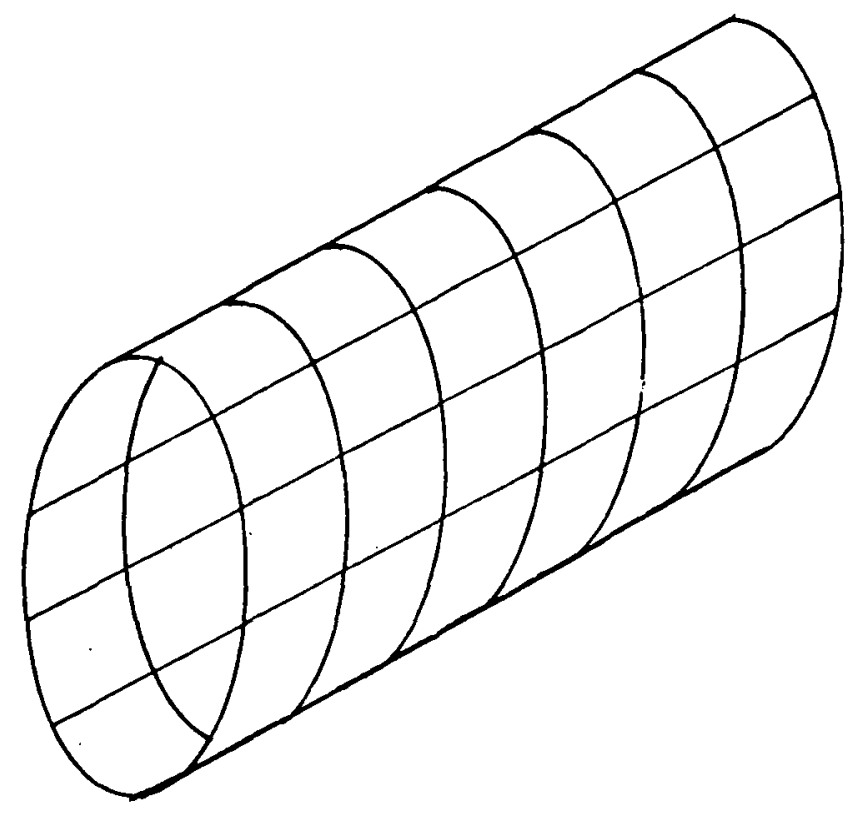

FIGURE 31 - PANELING OF AN INTERFERENCE SHELL

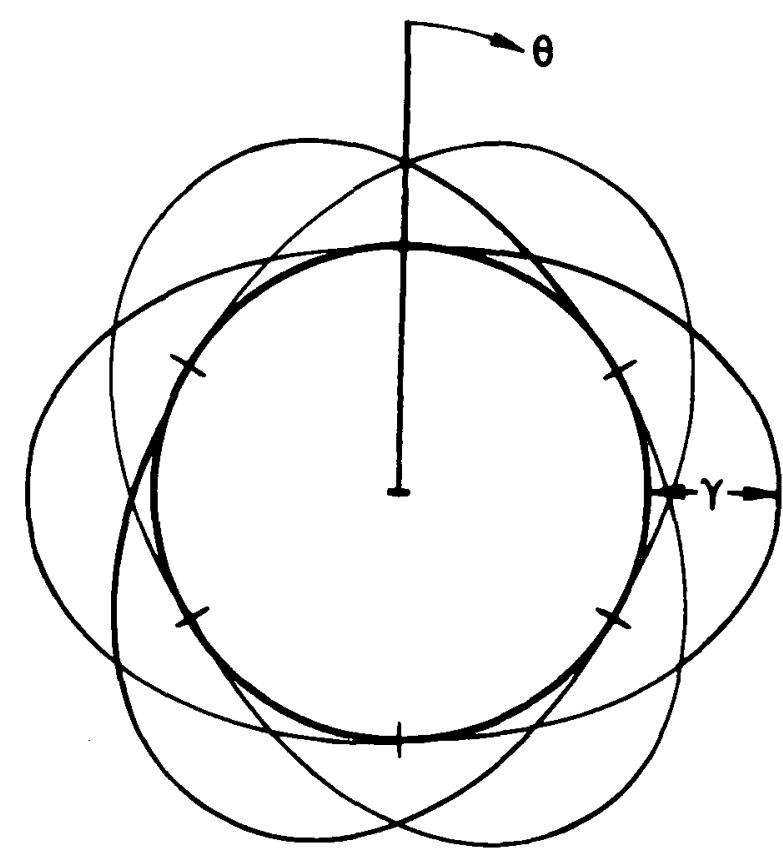

FIGURE 32 - THREE PANEL SPLINE FUNCTIONS ON INTERFERENCE SHELL 
already defined for planar panels. The analysis will be further restricted to circular cylinders having equal arc length panels.

The first step in the shell analysis is the derivation of the elementary horseshoe vortex on a circular cylinder. This singularity is obtained by integrating the doublet potential function (Ref. $5, \mathrm{pg}$. 87) from some initial point $x \circ$ to $\infty$ (downstream). This integration corresponds to a line of doublets from the point xo to $\infty$. The potential of the doublet with its axis in the $\overrightarrow{\mathbf{r}}_{\mathbf{0}}$ direction (Figure 30) is derived by differentiating the source potential (66) with respect to $\mathbf{r}_{0}$ :

$$
\Phi_{d}\left(x, r, \theta, x_{0}, r_{0}, \theta_{0}\right)=\frac{\partial \Phi_{g}}{\partial r_{0}}=\frac{\beta^{2}}{4 \pi} \frac{\left(r_{0}-r \cos \left(\theta-\theta_{0}\right)\right)}{\left[\left(x-x_{0}\right)^{2}+\beta^{2}\left(r^{2}+r_{0}^{2}-2 r r_{0} \cos \left(\theta-\theta_{0}\right)\right]^{3 / 2}\right.}
$$

The potential of the elementary horseshoe vortex is then:

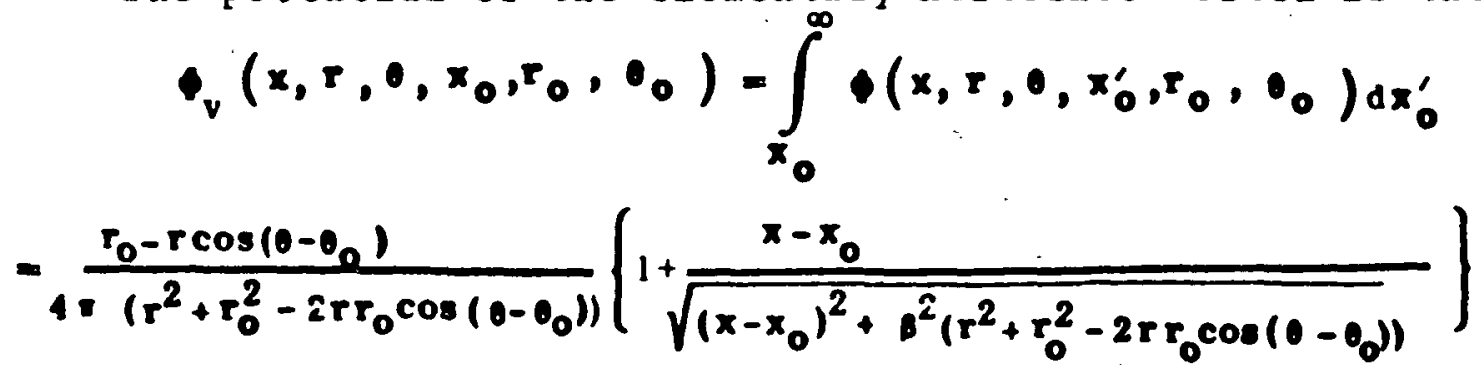

For this analysis, a spline arrangement.. spanning three circumferential panels was used (Figure 32). The circumferential variation can be derived using three quadratics (one on each panel) and a set of constraint requirements. On the first panel (Figure 33), the requirements are that the function must vanish in value and slope at the left edge. on the second panel the requirements are that the function be continuous in value and slope at the left edge. On the third panel the reguirements are that the function be continuous in value and slope at the left edge, and vanish in value and slope at the right edge. These eight requirements, coupled with the additional specifications of unit peak amplitude, completely determine the nine coefficients of the three quadratics composing a single spline. The spline distribution of bound vorticity (strength of elementary horseshoe vortex) can then be written as:

$$
Y_{j}(x, \theta)=\sum_{l=1}^{2} \sum_{m=1}^{3} x_{j}^{l} \theta_{j}^{m}
$$

where $x_{j}^{\prime}$ is tin same as that defined by Equations (26) and (27). and the $\theta_{j}^{m}$ for equal arc length panels are: 8.0 


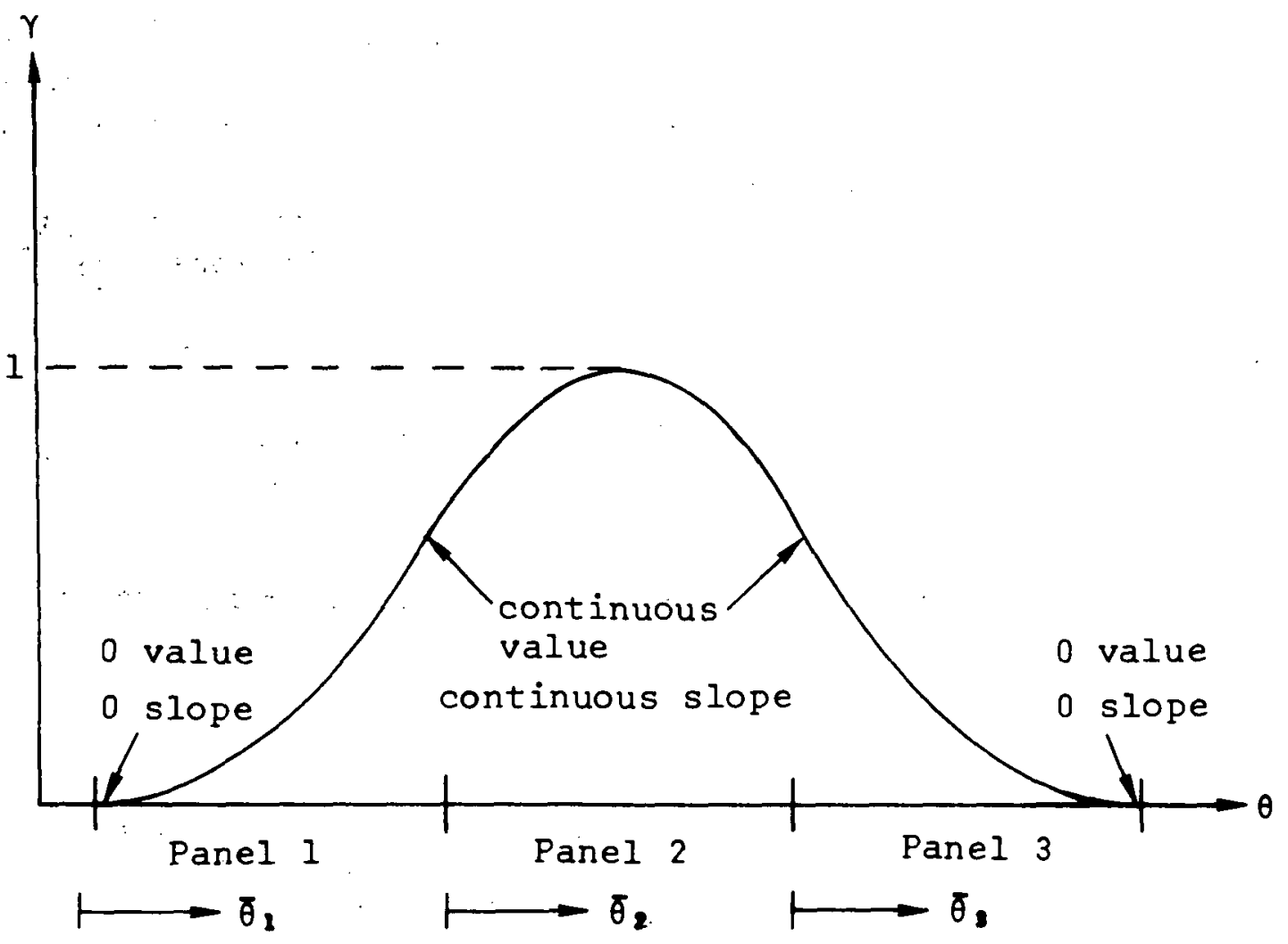

FIGURE 33 - BASIC THREE PANEL SPLINE 


$$
\begin{gathered}
\theta_{j}^{1}=\frac{2}{3} \bar{\theta}_{1}^{2} \\
\theta_{j}^{2}=\frac{2}{3}+\frac{4}{3} \bar{\theta}_{2}-\frac{4}{3} \bar{\theta}_{2}^{2} \\
\theta_{j}^{3}=\frac{2}{3}-\frac{4}{3} \bar{\theta}_{3}+\frac{2}{3} \bar{\theta}_{3}^{2}
\end{gathered}
$$

The $\bar{\theta}^{\prime}$ s are local coordinates for each panel ranging from 0 at the left edge to 1 at the right edge.

The influence coefficient $k \in[n \in l$ is found by differentiating Equation (68) with respect to $r$ (to find the normal velocity kerael) and evaluating this resulting expression on the surface of the cylinder ( $r$ equal $\left.r_{0}\right)$.

$$
K\left(x_{i}, \theta_{i}, x_{0}, \theta_{0}, x_{0}\right)=\frac{1}{4 \pi}\left\{\frac{1-2 \cos \Delta \theta}{2 r_{0}^{2}(1-\cos \Delta \theta)}\left[1+\frac{x_{1}-x_{0}}{R}\right]-\theta^{2} \frac{\left(x_{i}-x_{0}\right)(1-\cos \Delta \theta)}{2 R^{3}}\right\}
$$

Where $\Delta \theta$ is $\theta_{i}-\theta_{0}, R$ is $\sqrt{\left(x_{1}-x_{0}\right)^{2}+2 B^{2} r_{0}^{2}(1-\cos \Delta \theta)}$, and the subscript "i" denotes that the field point $(x, \theta, r)$ is on the cylinder surface.

The influence coefficient is then:

$$
A_{1 j}=\int_{Q_{L}}^{\theta_{R} x_{L E}} \int_{x_{L E}}^{x_{j}} Y_{j}(x, \theta) K\left(x_{1}, \theta_{i}, x, \theta\right) d x r d \theta
$$

The integration can be carried out in exactly the same manner as for the planar panels performing the $x$ irtegration first then using the numerical scheme, previously described, to integrate in the $\theta$ direction. This same rumerical integration scheme can be used since. the cylindrical kernel has the same singular properties as the planar kernel. 
For supersonic flow, the differences ir the cylindrical analysis are the same as outlined for the planar analysis basically the kernel and limits of integration change.

The supersonic source is (Ref. 5, pg. 87):

$$
\varphi_{0}\left(x, r, 0, x_{0}, r_{0}, \theta_{0}\right)=-\frac{1}{2 \pi} \frac{1}{\sqrt{\left(x-x_{0}\right)^{2}-\theta^{2}\left(r^{2}+r_{0}^{2}-2 r r_{0} \cos \left(\theta-\phi_{0}\right)\right)}}
$$

The joublet in the $\overrightarrow{\mathbf{r}}_{\mathbf{0}}$ direction is defined by $\phi_{\mathbf{d}}=\frac{\partial \mathbf{p}_{\mathbf{r}}}{\partial \mathbf{r}_{\mathbf{0}}}$

$$
\varphi_{d}\left(x, r, \theta_{0} x_{0}, r_{0}, \theta_{0}\right)=-\frac{\theta^{2}}{2 \pi} \frac{\left(r_{0}-r \cos \left(\theta-\theta_{0}\right)\right)}{\left[\left(x-x_{0}\right)^{2}-\theta^{2}\left(r^{2}+r_{0}^{2}-2 r r_{0} \cos \left(\theta-\theta_{0}\right)\right]^{3 / 2}\right.}
$$

and the horseshoe: vortex is defined by

$$
\theta_{v}=\int_{x_{0}}^{x-\sqrt{\theta^{2}\left(x^{2}+r_{0}^{2}-2 r r_{0} \cos \left(\theta-\theta_{0}\right)\right.}}
$$

$\phi_{v}\left(x, r, 0, x_{0}, r_{0}, \theta_{0}\right)=\frac{1}{2 \pi} \frac{r_{0}-r \cos \left(\theta-\theta_{0}\right)}{\left(r^{2}+r_{0}^{2}-2 r r_{0} \cos \left(\theta-\theta_{0}\right)\right)} \frac{x-x_{0} \sqrt{\left(x-x_{0}\right)^{2}-\theta^{2}\left(r^{2}+r_{0}^{2}-2 r r_{0} \cos \left(\theta-\theta_{0}\right)\right)}}{20}$

In the above $B^{2}$ is defined as $M^{2}-1\left(B^{2}\right.$ is always fositive whether supersonic or subsonic).

The kernel nezded for the influence coefficient is derived from (77) by differentiatirg with respect to $r$ and evaluating the result on the surface of the cylinder ( $r$ equal $r_{0}$ ):

$$
K\left(x_{1}, \theta_{1}, x_{0}, \theta_{0}, r_{0}\right)=-\frac{1}{2 \pi}\left[\frac{1}{2 r_{0}^{2}(1-\cos \Delta \theta)} \frac{\left(x_{1}-x_{0}\right)}{R}-\beta^{2} \frac{\left(x_{1}-x_{0}\right)(1-\cos \Delta \theta)}{2 R^{3}}\right]
$$

where $\Delta \theta$ is $\theta_{1}-\theta_{0}$ and $R$ is

$$
\sqrt{\left(x_{1}-x_{0}\right)^{2}-2 \theta^{2} r_{0}^{2}(1-\cos \Delta \theta)}
$$

The limits of integration for the supersonic integral are found by determining the intersection of the forward Mach cone, emanating frcm the control point $\left(x_{1}, \theta_{f}\right)$, with the cylinder. 
The equation of the Mach cone evaluated on the surface of the cylinder is:

$$
\left(x-x_{i}\right)^{2}=2 \theta^{2} x^{2}[1-\cos (\theta-\theta)]
$$

For the simple panels used in the analysis, the intersections are found by replacing $x$ with $x_{L v}$ ( $x$ of the leading edge) and $x_{\text {TE }}\left(x\right.$ of the trailing edge) ${ }^{-}$Equation $(79)$ then gives the $\theta$ values of the intersections which can be used in the integration limit scheme previously described to establish the region 10.1 , or II) and set the limits for the $\theta$ integration.

Once the influence coefficients are computed, the boundary conditions are satisfied and remaining analysis is performed using the same logic as was used for the planar development. The boundary conditions of course are different for the interference sell as mentioned before and are more fully covered in Reference 1. However, this difference does not affect the mechanics of obtaining a solution, it only changes the layout of the overall influence coefficient matrix in that the body contained within the shell is assumed to have no influence on the shell.

The above analysis could be repeated using doublets, but since the equivalent doublet strength is the integral of the bound vortex strength in the $x$ direction (Ref. 5, pg. 135), such a formulation would require quadratic distributions in both $X$ and $Y$. This arrangement could be made using the relations given by Equations (70) through (72) for both the $X$ and $\theta$ variations. An influence coefficient scheme could then be formulated which would not shed vorticity. This scheme could then be compared with the vortex scheme to determine the merits of each for body representation. The scope of the present development did not include such a comparison. 


\subsection{SECOND ORDER SOLUTIONS USING THE VORTEX SPLINE}

In the introduction it was pointed out that one of the purposes for developing a new aerodynamic technique was to have a scheme wich would calculate those lateral-directional stability derivatives which cannot bc handled by tha current formulations which itreat arbitrary wing-body combinations. The previous sections have shown the development of a scheme which was felt would provide the necessary improvements to meet the lateraldirectional rejuirements. The course of develofment was to be an initial treatment of planar wings (Bef. 13), then a treatment of bodies in the presence of wings (the interference shel1). and finally the treatment of these wings and rodies and their combinations in interaction flow. The initial interaction flov studied was for angle of attack-angle of sideslif.

The basis for the second-order flov analysis is given in keference 4. In essence, the development assumes that the exact nonlinear, potential flow equation can be expanded in terms of small geometric parameters which define the problem (E.g.. angle of attack, ângle of sideslip, wing thickness, etc.l. When this expansion is carried out, an ordered set of linear equations is obtained. Fach of these equaticns has its own boundary conditions which are determined by a similar expansion of the exact boundary conditions. The higher crder equations (those involving powers and products of the small parameters) have non-homogeneous differential equations while the first order equations have the fariliar linearized, homogeneous differential equation, e.g.,

$$
\begin{gathered}
\left(1-M^{2}\right) \frac{\partial^{2} \phi_{2}}{\partial x^{2}}+\frac{\partial^{2} \phi_{2}}{\partial y^{2}}+\frac{\partial^{2} \phi_{2}}{\partial z^{2}}=F(x, y, z) \\
\left(1-M^{2}\right) \frac{\partial^{2} y}{\partial x^{2}}+\frac{\partial^{2} y}{\partial y^{2}}+\frac{\partial^{2}}{\partial z^{2}}=0
\end{gathered}
$$

where the subscripts " $1 "$ and "2" denote the first and second order terms from the potential flow expansion. The expansion assures that $F(x, y, z)$ is only a function of $\phi_{1}$ and its derivatives.

The forms of these results (Equations (80) and (10)) are standard ones arising from expansions so that the technique used to evaluate results for these equations is general. This point is being made because the expansion in Reference 4 did not properly account for all the terms present and therefore is not valid. Although the result is not correct, the forms of the equations are indicative of the types of numerics needed. The work done in 
implementing the technique is therefore valid and would be applicable wen the proper expansion is found. Because of this. the technique used to solve the class of problems represented by Equation (80) is presented, but the presentation will be somewhat yeneral in nature. Detailed extension of the schede to second order ving-body configurations vas of course not done.

The solution to Equation ( 80 ) consists of two parts - a homogeneous part, $\phi_{2 h}$. and a particular part, $\phi_{2} p$. Since Equation 10 can be added to Equation (80) in any proportion without changing the problem, an assumption is made that a $\phi_{2} p$ can be constructed uhich will satisfy the vake coundary conditions. This leaves homogeneous boundary conditions in the vake for the homogeneous solution $\phi_{2 H}$. This means that the same homogeneous formulation. (i.e.. vortex spline) can be used for both first and second order homogeneous solutions. The particular solution.

$\phi_{2 P}$. provides a basic velocity kernel in exactly the same way the horseshoe vortex (homogenecus solution) provided the velocity kernel for the wing and the interference shell (Equation (68)). Folloving the procedure outlined for the shell, this potential function for $\phi_{2 P}$ is differentiated with respect to the surface normal at the control point. The resulting differentiated yuantity is the normal velocity kernel, analogous to those associated ith the first crder problems for wings and interference shells (Equations (12) and (73), respectively).

Unlike the first order problea the particular solution does not depend on matching the boundary conditions. Rather, the strengths of the distributions and the distributions themselves are known directly and no solving is involved. This is because $F(x, y, z)$ is only a function of the tirst order problem, whose strength is determined initially in the solution to that problex. Specifically then, the velocity kernel determined from $\phi_{2 p} p$ is used to compute an additional influence on the boundary conditions and not used as a means to satisfy them. The downashes conputed from the particular kernel and those obtained from the boundary condition expansion are combined to provide a nev set of boundary conditions for the second crder bomogeneous solution. The homogeneous solution, $\phi_{2} H$, is then used to satisfy the combined boundary conditions. The net result is that the combination of $\phi_{2 P}$ and $\phi_{2 H}$ satisfies the boundary conditions for the second order problem uich vere determined by the initial expansion.

Since the second order homogeneous differential equation is identical to the first order equation, the same basic solution technique can be used (i.e. the vortex spline). The second order houogeneous solution, of course, has different boundary conditions and correspondingly different strengths from that of the first order. Also, depending on wich equation is involved, there can be different symetry requirements for the solution (e.g. angle of 
dttack -angle of sideslip froblem assuming zero sideslip is antisymmetric while the first order problem, angle of attack, is symmet.ric).

Figure 34 shows how a seccnd order problem could be solved using the vortex spline scheme. Basically the central block of logic is the homogeneous boundary conditicn solver which is the vortex spline scheme (Figure 21). The secord order particular solution section of the logic blcck frovides the downwashes vhich must be combined with the specified boundary condition (from the expansion). These downwashes then represent a new set of boundary conditions for the homogeneous solution ( $\phi_{2} \mathrm{H}$ ) vhich is solved in the exact same manner as the first order problem. The quantities necessary to obtain the pressures are then comfuted (this is not alvays just the bcund component of vorticity as pointed out in Reference 4) and the stability derivatives are obtained.

The advantage of the vortex spline for solving these second order problems can be seen from a study of the characteristics of the forcing functions $F(x, y, z)$ (Ref. 4). These functions generally involve derivatives of the first order solution. A solution to the first order froblem must, therefore, be capable of providing sufficient derivative resolution that the second order problem can be solved accurately. The splines provide this capability as can be seen from Figures 22 through 28 . 


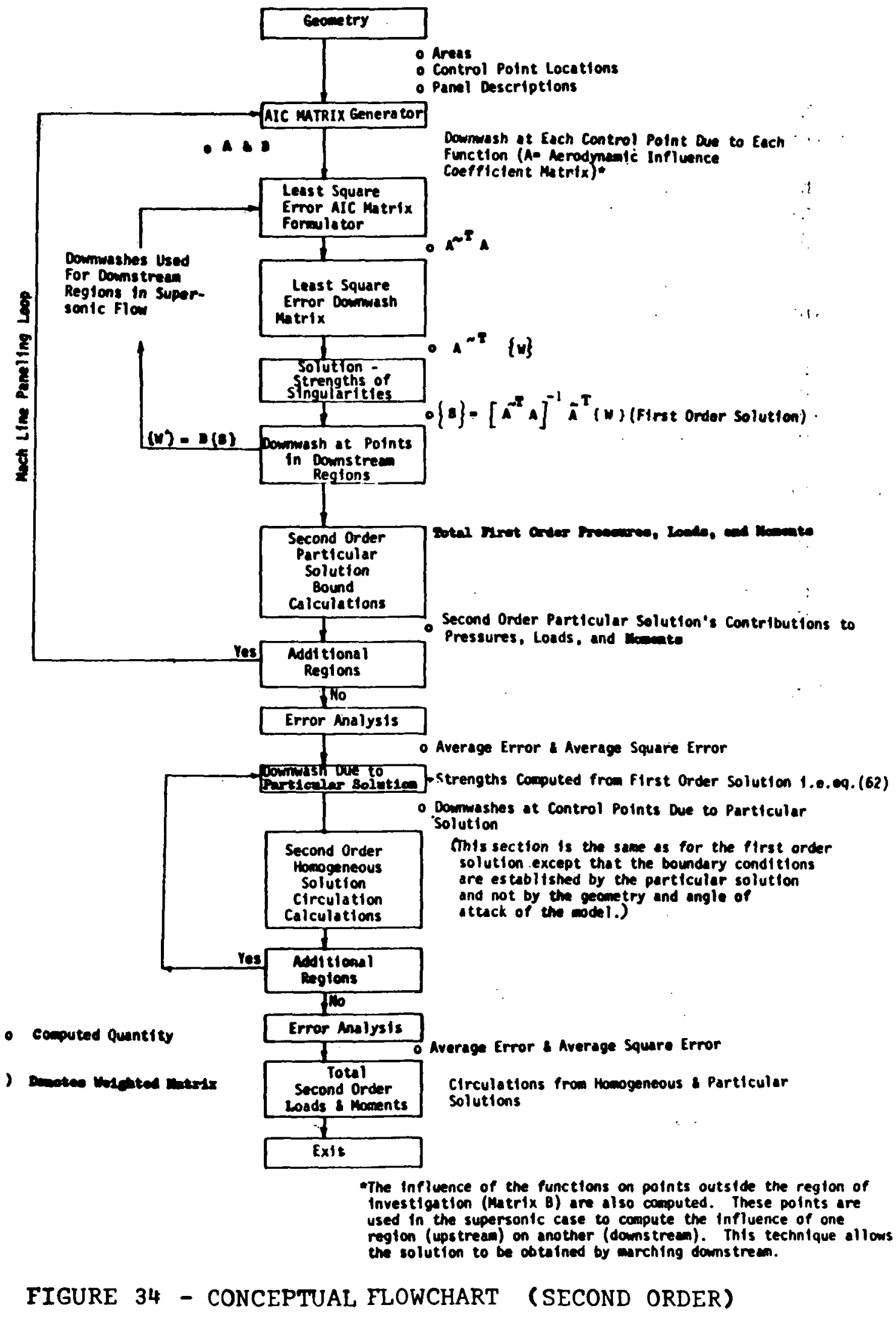




\subsection{CONCLUDING REMARKS}

The experience gained vith the portex spline scheme has indicated the following:

1. The solution is not strongly dejendent on paneling arrangement; it works well with uniform or nonuniform panel spacing.

2. The scheme works equally well for both subsonic and supersonic flow.

3. Special Mach line paneling produces better pressure resolution in supersonic flow than geometric paneling. However, in all cases the gecmetric paneling produced accurate values for lift and moment.

4. The absence of dounush singularities in the rake lexcept behind planform tips) imflies that tails can be paneled independently frow wings.

5. Computation times are small, requiring only a few seconds (CDC 6600) for simple flanforms and less than 2 minutes for finely panelea planforms.

6. Accurate results can be obtained with sparse paneling.

7. The extension of the scheme to the representation of arbitrary configuration surfaces appears entirely feasible.

8. The rumerical stability of the scheme provides flexibility in the application of the method. such stability is a fundamental requirement for the computation of downash (camber slope) given pressure (this type of problem solving is termed the design mode).

9. The scheme is self-checking in that the user can judge the accuracy of the numerical representation by simply examining the root mean square ercor in the downwash. (This rms value seems to be the same order of magnitude as the rms the pressure distribution for the cases tested sc far.l.

Future plans for the scheme should include investigation of an automatic paneling techrique wherein the user would only be required to input the vehicle shape and specify an acceptable rms error. The progran would then automatically panel the configuration, solve the problem, examine the Ims error, and repanel based on the error. This cycle would continlie until the specified ras error was achieved. Such a development would lead 
to' a self-checking scheme that provides results of a predetermined level of numerical accuracy. 


\subsection{REFERENCES}

1. F. A. Hooduard, "A Method of optimizing Camber surfaces for Hing-Bod 7 Combinations at Supersonic Speeds, Part 1 - Theory and Application". D6-1074\%, The Boeing Company, prepared under NaSA contract NAS2-2282, 1965.

2. H. S. Rove, "Collocation Hethod for Calculating the Aerodynamic Pressure Distributions on a lifting Surface oscillating in Subsonic Conpressible Flou",Aara Syaposium on Structural Dynamics and Aeroelasticity.Boston, Uassachusetts, August, 1965.

3. A. R. Dusto and P. P. Polentz, "The PlexSTaB stability and Control Computer Program for Flexible Aircraft". Integrated Program for Aerospace Vehicle Design (IPAD) Colloquiua, langley Besearch Center, Oct. 2-4, 1972.

4. P. E. Rubbert, "Sideslip of Wing-Body Combinations", D660160 , The Boeing Company, prepared under NASA Contract NaS 25006 . Day 1972 .

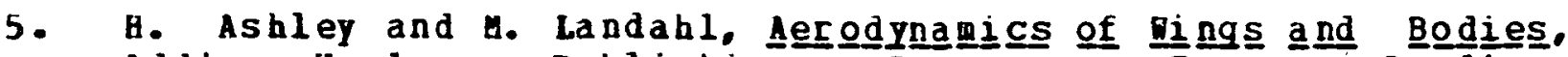
Addison-Wesley Publishing Company. Inc.. Reading. Massach usetts, 1965.

6. R. L. Bisplinghoff, H. Ashley, and R. L. Halfman, Aeroelastícity. Addison-Wesley, Reading, Massachusetts, $195 \mathrm{~b}$.

7. K. Mangler. "I aproper Integrals in Theoretical Ae rodynancs", British A. R. C., RE M $2424,1951$.

8. R. Church, "Numerical Quadrature Studies", Sperry Band Research Beport, SRRC-RR-64-99, Decenber, 1964.

9. P. Businger and G. H. Golub, "Linear Least Squares Solutions by Householder Transformations". Handbook Series Iinear Algebra, Numerische athematik, Vol. 7. pp. 269-276, 1965.

10. B. van Spiegel, "Boundary Value Problems in litting Surface Theories", Verslagen en Verhandelingen, 22, vational Luchtraart laboratorium, Amsterdam, 1959.

11. R. T. Jones and Doris Cohen, Hiqh Speed uing Theory. Princeton University Press. Princeton, New Jersey, 1960 .

12. Hsue-Shen Tsien, "Supersonic Flow Orer an Inclined Body of Revolution", Journal of the Aeronatical Sciences, pp. 480$483,1938$. 
13. J. E. Mercer, J. A. Meber, and E. P. Lesferd, "Aerodynasic Intluence Coefficient rethod using singularity splines". Paper No. 73-123, AIAA 11th Aerospace Sciences Heeting, Washington D. C.. Jan. 10-12, 1973.

14. J. H. B. Suith. J. A. Beasley, Diana Short and P. Malkden. "The Calculation of the Harp to Produce a Given Load and the Pressures due to a Given Thickness on $T$ hin Slender Wings in Supersonic Flow". British A. B. C.. R E H 3471 . 1965. 


\subsection{APPENDIX}

\section{Doublet Characteristic Box Method}

This method is an alternate approach to the vorter spline in supersonic flow. The method showed promise but further development was halted when it became evident that a great deal of tise would be needed to calculate all the integrals involved for the various building blocks, as well as developing a systematic approach to identify all the "special" Mach lines. There was. however. substantial vork done on the fundamentals. This effort is presented below.

The first step in the present work was to identify the "special" Mach lines emanating from planform edge breaks. These lines are used to divide the planform into several different regions. Each of these regiors is divided into a network of panels bounded by Hach lines as shoun in Figure 35.

The basic building blocks comprising the method were identified. These blocks, along with their contrcling parameters, are shown in Figure 36. The building blocks include (see Figures 35 and 36 ):

$\begin{array}{ll}\text { I } & \text { Hegular - Bounded by Mach Lines } \\ \text { II } & \text { Two Subsonic Leading Edges } \\ \text { III } & \text { One Subsonic Leading Edge } \\ \text { IV } & \text { Supersonic Leading Edge } \\ \text { V } & \text { Subsonic Trailing Edge } \\ \text { VI } & \text { Hake }\end{array}$

A planform is represented by a superposition of these building blocks. The singularity distribution on each panel is assuned to be a function cf the Mach line coordinates ( $r, s$ ) Figure 37. The type of singularity distribution that was explored initially was a doublet distribution of the form

$$
\Delta s=a+b r+c r^{2}+d s+e s^{2}+f r s+g r^{2} s+h r s^{2}+k r^{2} g^{2}(81)
$$

where $\Delta \phi$ is the velocity potential jump across a panel and $a, b, \ldots, k$ are unknown coefficients. This distribution provides vorticitg components that vary linearly and quadratically with $r$ and $s$ as shorn in Pigures 38 and 39 , and insure smooth derivatives of the resulting first order solutions. 


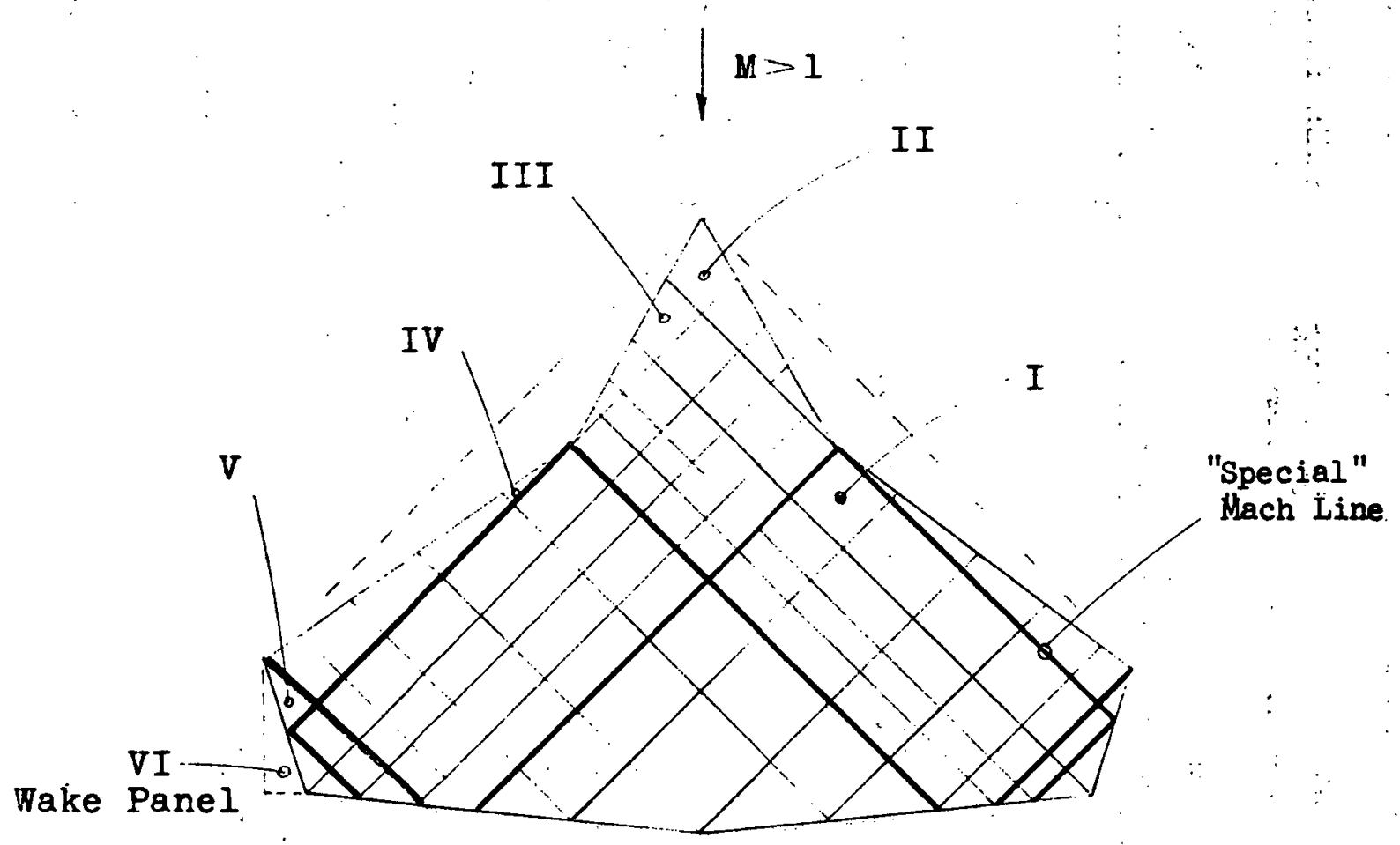

FIGURE 35 - PANELED PLANFORM 


\begin{tabular}{|c|c|c|c|c|c|c|}
\hline 怘 & 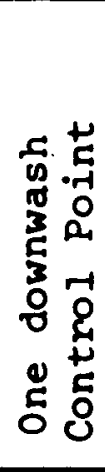 & 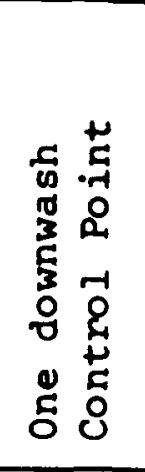 & 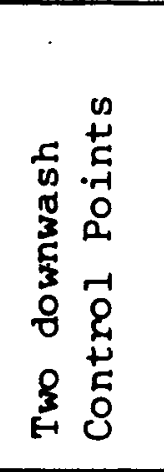 & 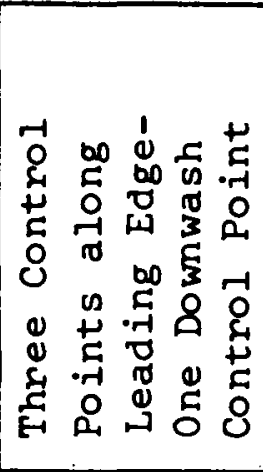 & 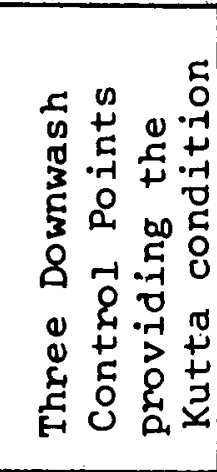 & 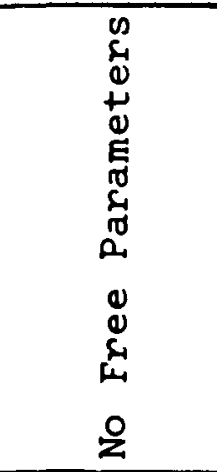 \\
\hline 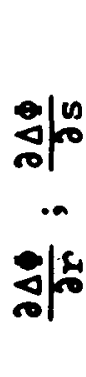 & 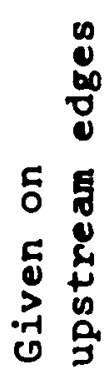 & & 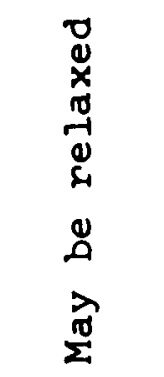 & & 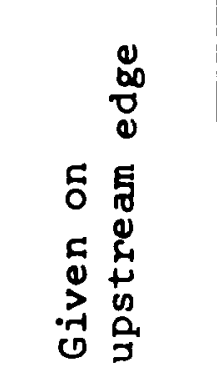 & \\
\hline 8 & 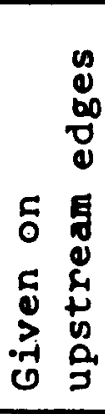 & $\begin{array}{l}\infty \\
0 \\
\infty \\
0 \\
0 \\
c \\
0 \\
0 \\
0 \\
0 \\
N\end{array}$ & 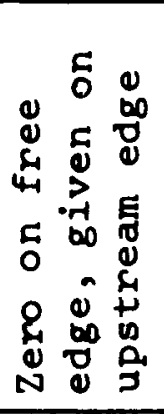 & 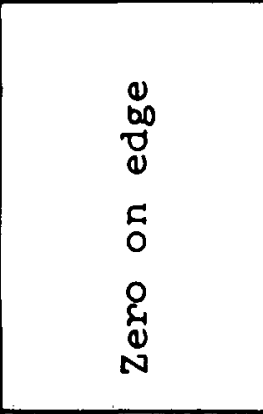 & 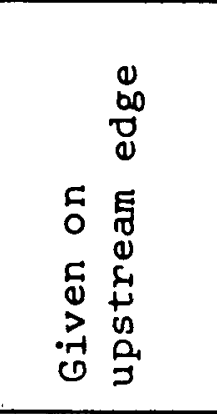 & 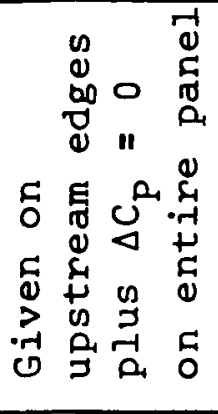 \\
\hline 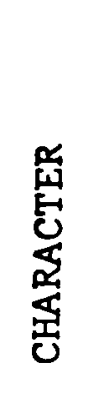 & 4 & \& & & & us & $\underset{8}{\infty}$ \\
\hline 㟧 & 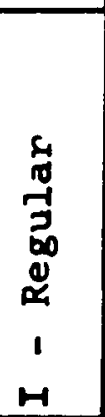 & 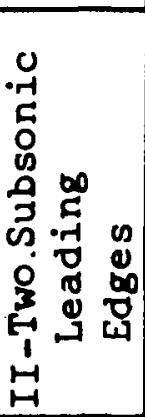 & 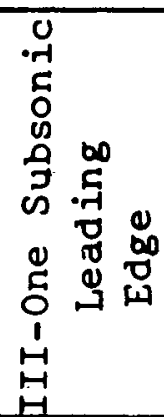 & 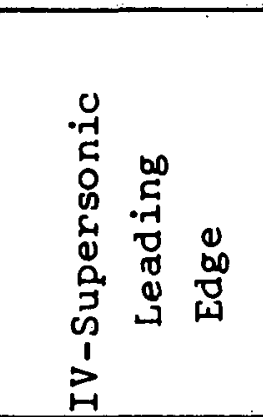 & 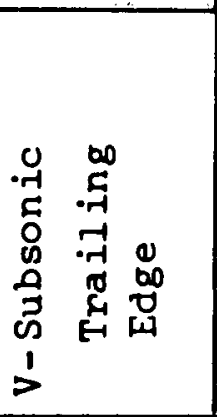 & $\begin{array}{l}\stackrel{y}{y} \\
\frac{\pi}{3} \\
\frac{3}{1} \\
\stackrel{H}{S}\end{array}$ \\
\hline
\end{tabular}

号 


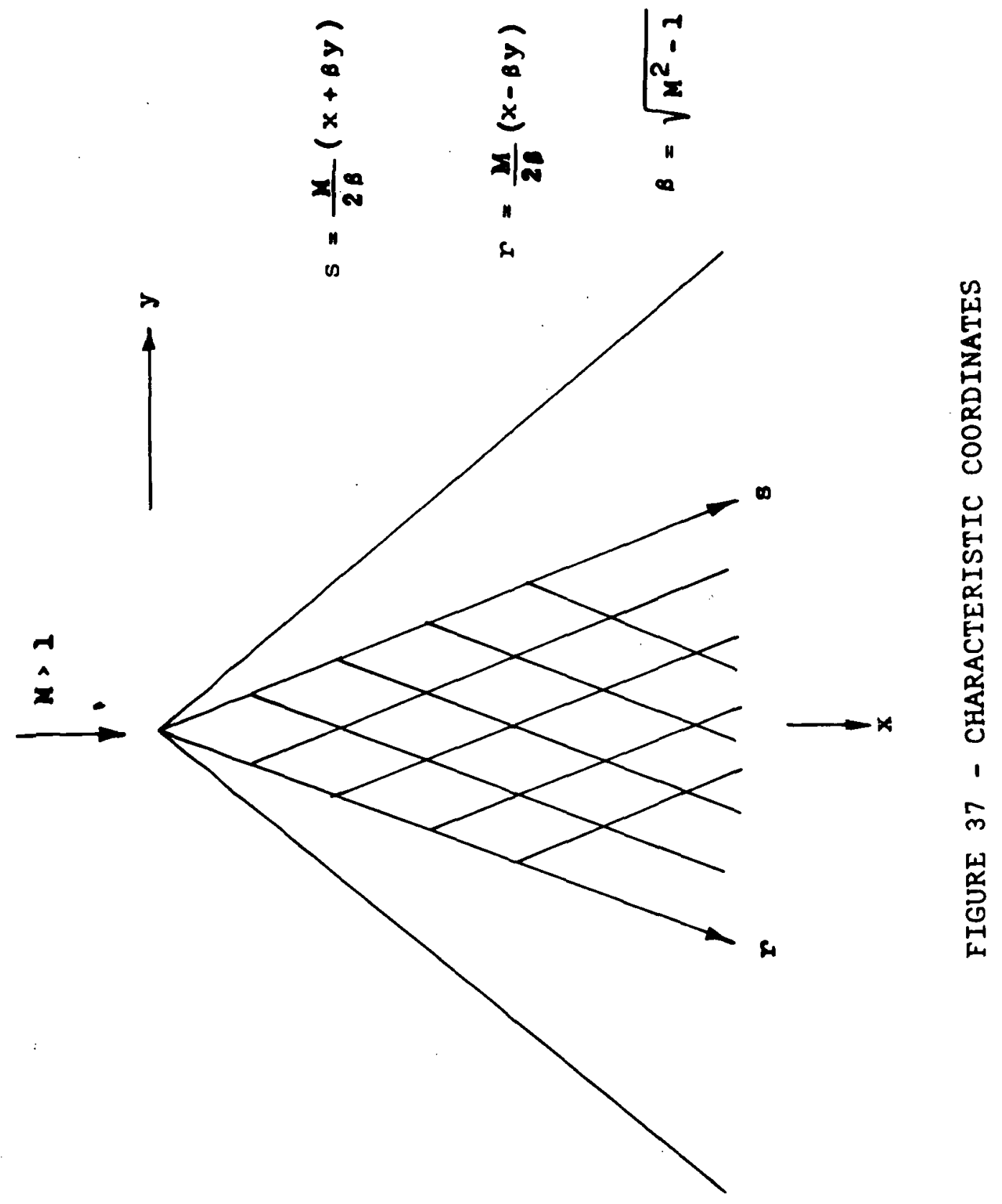




$$
\left.\right|_{x}
$$

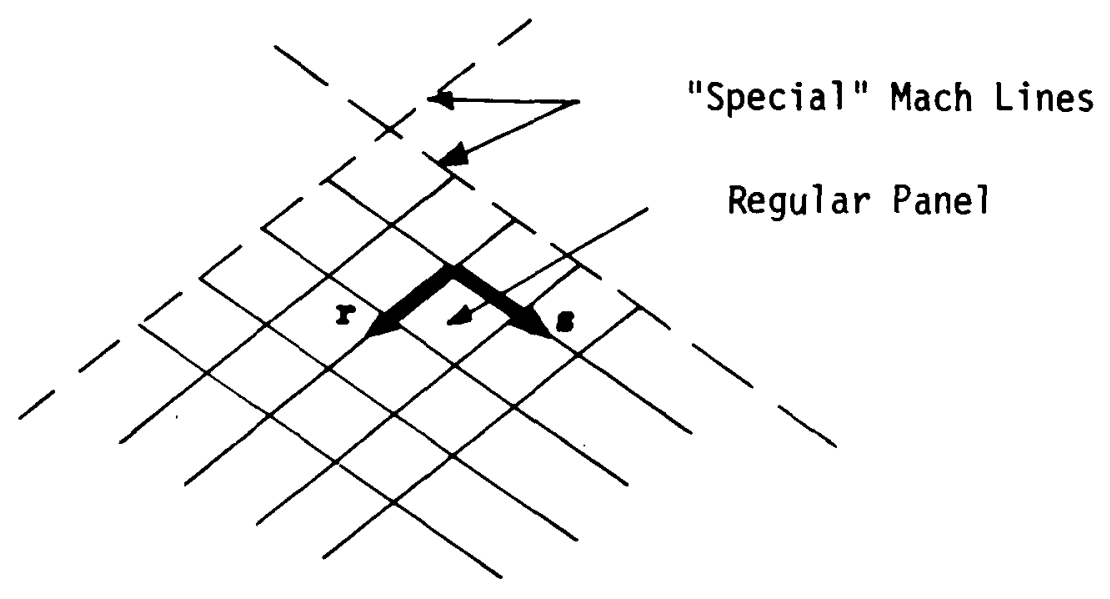

FIGURE 38 - INTERIOR REGION PANELING

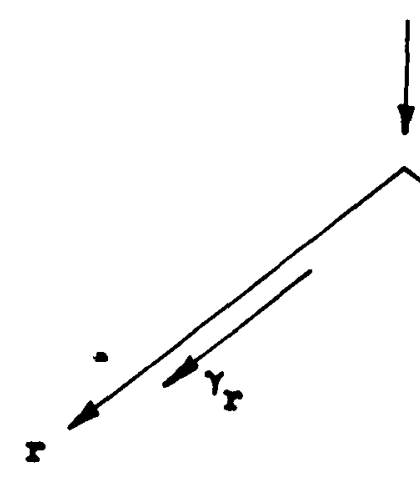

$$
r_{\mathbf{B}}=\frac{\partial \Delta}{\partial \mathbf{r}} \rightarrow \begin{cases}r_{\mathbf{B}} & \text { varies linearly with } r \\ r_{\mathbf{B}} & \text { varies quadratically with } s \\ r_{\mathbf{r}} & \text { varies linearly with } s \\ r_{\mathbf{r}} & \text { varies quadratically with } r\end{cases}
$$

FIGURE 39 - BASIC VORTICITY DISTRIBUTION 
The unknown coefficients are found in the following ways: the solution starts with a leading edge panel (II or IV Pigure 36) for which $\Delta$ is known ( 0 at leading edge): the solution then proceeds downstream by matching at comon edges. These matching conditions consist of requiring $\Delta$, , $r_{r}$, and $r_{8}$ (Figure 39) to be continuous across the panel edges. This leaves one free parameter remaining for each panel which is deternined by the downwash boundary conditions. The basic method of analysis is shoun in Eigure 40 for the "Regular" panel. The procedure consists of matching $\Delta \phi$ and the two derivatives, $\partial \Delta \phi / \partial \mathbf{r}$ and $\partial \Delta \phi / \partial \theta$, along the two upstream edges. This leaves only the parameter $k$ as unknown. $k$ is then determined by satisfying the downash boundary condition at a control point placed on the panel.

Along the network boundaries (either "special" Mach lines or planform edges), some of the matching conditions (i.e... $\Delta \phi, \partial \Delta \phi / \partial r$ and $\partial \Delta \phi / \partial s)$ are relaxed. They are replaced by additional downush boundary conditions to provide the freedow needed to account for loading discontinuities, Kutta conditions or other constraints. Although the above procedure frovides a determinate set of equations, a minimum error technique might also be incorporated to enable multiple boundary points cn the panels.

The influence coefficients relate potential jump, $\Delta \phi$, to the dounwash, a. through the planar relationship (the basic doublet representation - Ref. 14).

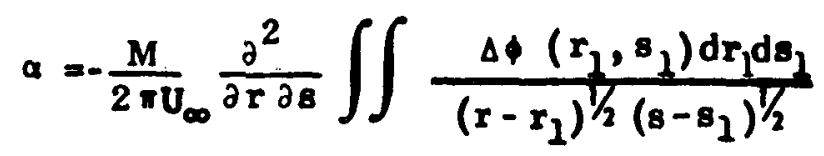

Here is the Mach numer and $\|_{\infty}$ is the free stream velocity. of:

Thus, the unknown coefficients are determined by combinations

- Downwash (camber)

- Matching - $\Delta \uparrow, \frac{\partial \Delta t}{\partial r}, \frac{\partial \Delta \phi}{\partial s}, \Delta C_{p}$

- Miniaum Error

An investigation of the varicus building blocks and the matching conditions provides the results shown in Figure 36 . This figure illustrates the matching conditions applied and the number of resulting free parameters, any of which might be relaxed. Note that a wake panel is completely specified (no tree parameters) by the upstream conditions. 


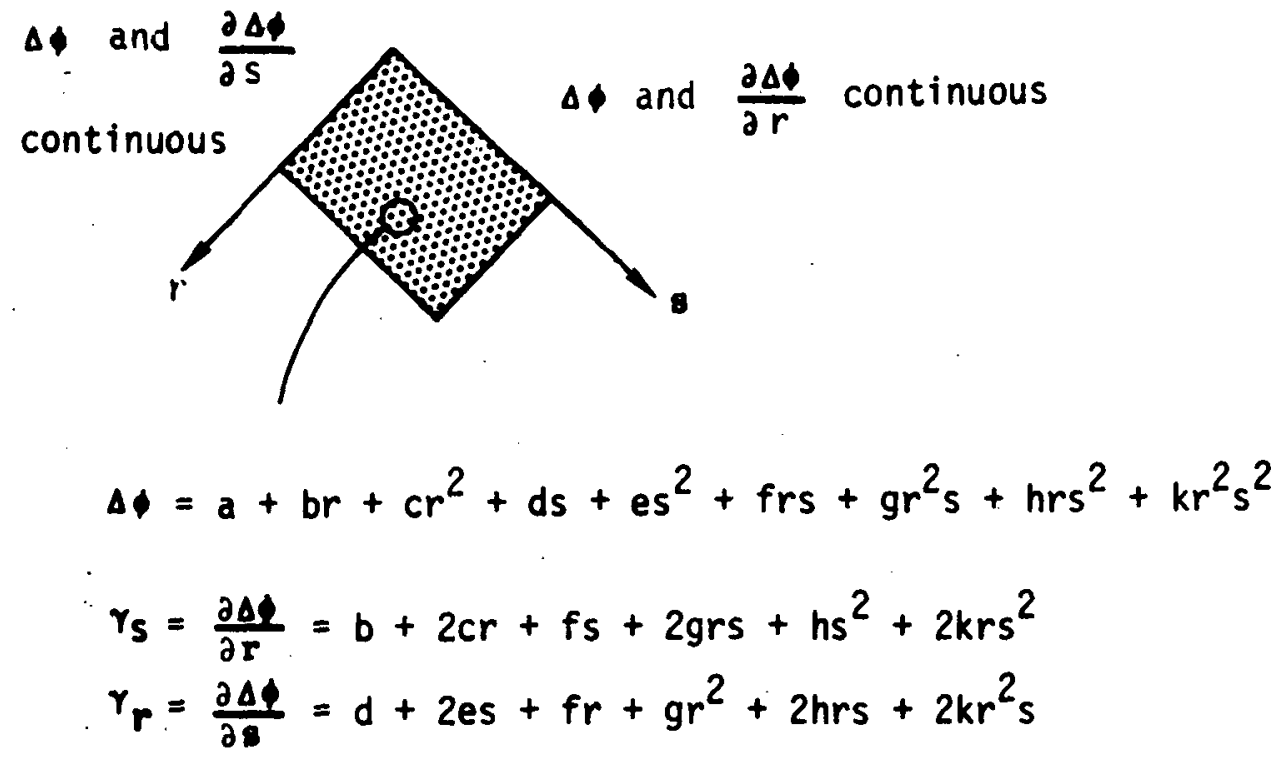

1) At $r=0, \Delta$ given, $a+d s+e s^{2}$ matches the upstream quadratic function $\therefore a, d, e$ determined

2) At $s=0, \Delta$ given $\therefore \quad b$ and $c$ determined as in 1)

3) At $r=0, \frac{\partial \Delta \phi}{\partial r}$ given, $b+f s+h s^{2} \therefore f, h$ determined as in 1)

4) At $s=0, \frac{\partial \Delta l}{\partial g}$ given, $d+f r+g r^{2} \therefore g$ determined as in 1)

5) unknowns determined from the downwash constraint

$$
\alpha \simeq \iint_{\Delta \phi K}\left(r, r_{1}, s, s_{1}\right) d r_{1} d s_{1}
$$

where $K\left(r, r_{1}, s, s_{1}\right)$ is the kernel from equation 82 . 
With the sideslip problem in view, the following basic steps vere used to make up the doublet characteristic box method.

1) The functional form for the potential jump was selected (i.e.. Equation (81)).

2) The boundary conditions are used to drtermine the parameters of the doublet distribution through combinations of known downash (camber), matching conditions and methods of minimum error.

3) a) Pressure (and ensuing load) can be found directly as the spatial derivative (in the free strea direction) of the potential jump.

b) Second order sideslip calculations are made possible by the representations of the velocity potential through the improved spatial derivatives of the first order potential function.

The complexity of the doublet characteristic box wethod hinges on the downyash-potential jump relationship which must be integrated analytically, numerically, or a combination of the two. Accordingly, the basic downuash-potential jump relationship might be cast into a more suitable for than presented by Equation (82) - such a form might carry the double differentiation under the integral sign, for example.

An investigation of the various building blocks and the matching conditions provides the results shown in fiqure 36 . 
"The aeronautical and space activities of the United States sball be conducted so as to contribute. . . to the expansion of human knowledge of phenomena in the atmosphere and space. The Administration shall provide for the widest practicable and appropriate dissemination of information concerning its activities and the results thereof."

\section{NASA SCIENTIFIC AND TECHNICAL PUBLICATIONS}

TECHNICAL REPORTS: Scientific and technical information considered important, complete, and a lasting contribution to existing knowledge.

TECHNICAL NOTES: Information less broad in scope but nevertheless of importance as a contribution to existing knowledge.

TECHNICAL MEMORANDUMS:

Information receiving limited distribution because of preliminary data, security classification, or other reasons. Also includes conference proceedings with either limited or unlimited distribution.

CONTRACTOR REPORTS: Scientific and technical information generated under a NASA contract or grant and considered an important contribution to existing knowledge.
TECHNICAL TRANSLATIONS: Information published in a foreign language considered to merit NASA distribution in English.

SPECIAL PUBLICATIONS: Information derived from or of value to NASA activities. publications include final reports of major projects, monographs, data compilations, handbooks, sourcebooks, and special bibliographies.

\section{TECHNOLOGY UTILIZATION}

PUBLICATIONS: Information on technology used by NASA that may be of particular interest in commercial and other non-aerospace applications. Publications include Tech Briefs, Technology Utilization Reports and Technology Surveys.

Details on the availability of these publications may be obtained from:

SCIENTIFIC AND TECHNICAL INFORMATION OFFICE 